TRANSACTIONS OF THE

AMERICAN MATHEMATICAL SOCIETY

Volume 187, Issue 1, 1974

\title{
ON SYLOW 2-SUBGROUPS WITH NO NORMAL ABELIAN SUBGROUPS OF RANK 3, IN FINITE FUSION-SIMPLE GROUPS
}

\author{
BY
}

\author{
ANNE R. PATTERSON
}

\begin{abstract}
Let $T$ be any finite 2-group which has a normal four-group but has no normal Abelian subgroup of rank 3, and assume $T$ is not the dihedral group of order 8. If $T$ is a Sylow 2-subgroup of a finite fusion-simple group $G$, it follows (Thompson) from Glauberman's $Z^{*}$-theorem that $T$ has exactly one normal four-group, say $W$. This paper establishes what isomorphism types of $T$ can so occur under the hypothesis that $N_{G}(T)=T C_{G}(T)$ and the three nonidentity elements of $W$ are not all $G$-conjugate. All $T$ arrived at in this paper are known to so occur.

The reason for this hypothesis is that the similar situation for $T$ with a normal four-group and no normal Abelian subgroup of rank 3 , where $T$ is a Sylow 2-subgroup of a finite simple group $G$ but without the above hypothesis, had been analyzed earlier by the author (under her maiden name, MacWilliams; Trans. Amer. Math. Soc. 150 (1970), 345-408).
\end{abstract}

The result of Feit and Thompson [7] suggests that one might classify finite simple groups by their Sylow 2-subgroups. One measure of the complexity of the possible 2-structure of a group is its normal 2-rank, i.e., the largest rank of an Abelian 2-subgroup which is normal in some Sylow 2-subgroup containing it. If the normal 2-rank is 1, then the Sylow 2-subgroup is cyclic or of maximal class (Blackburn [3]), and the simple groups with normal 2-rank 1 are known (Brauer, Gorenstein, Walter, Alperin [4], [9], [2]). The present paper is a completion of [12], in which a start was made on finding the 2-groups $T$ that can occur as Sylow 2-subgroups of simple groups $G$ with normal 2-rank 2; except that in the present paper we actually assume only that $G$ is fusion-simple, i.e., that $G$ has no proper 2-quotient, no nonidentity normal subgroup of odd order, and no nonidentity central elements.

We use the following result of Thompson, of which a proof is given in [12]: If $G$ is a fusion-simple group with normal 2-rank 2, then the Sylow 2-subgroups

Received by the editors September 11, 1972.

AMS (MOS) subject classifications (1970). Primary 20D05, 20D20; Secondary 20D15. Key words and phrases. 2-group, Sylow 2-subgroup, rank, fusion-simple, conjugate, transfer homomorphism, fusion.

\footnotetext{
Copyriaht $\odot 1974$, American Mathomatical Society
} 
of $G$ are either dihedral of order 8 or have precisely one normal four-group. The 2-groups which can arise will be found by considering the various possibilities for the $G$-fusion of the involutions of this four-group.

In view of [12], we shall assume the following:

Hypothesis. $T$ is a 2-group with normal 2-rank 2; $T$ is a Sylow 2-subgroup of a fusion-simple finite group $G ; N_{G}(T)=T C_{G}(T) ; T \nRightarrow D_{8}$; and the involutions of the unique normal four-group $W$ of $T$ are not all $G$-conjugate.

We shall obtain the following results under this hypothesis:

Theorem A. Suppose that the involutions of $W$ fall into two G-classes. Then one of the following bolds:

(a) $T \simeq D_{2^{m}}\left\{\mathrm{Z}_{2}\right.$, where $m \geq 4$.

(b) $T=\left\langle\langle a, \lambda\rangle \times\langle\beta, \mu\rangle, \pi, r: a^{2}=\lambda^{2^{n}}=\beta^{2}=\mu^{2^{n}}=1, a \lambda a=\lambda^{-1}, \beta \mu \beta=\right.$ $\mu^{-1} ; \pi^{2}=\lambda \mu, \pi$ centralizes $\lambda$ and $\mu,[\alpha, \pi]=\lambda,[\beta, \pi]=\mu ; \tau^{2}=1, \alpha^{\tau}=\beta, \lambda^{t}$ $\left.=\mu, \pi^{\top}=\pi\right)$, where $n \geq 2$.

(c) $T \cong D_{2^{m}}^{+}\left\{\mathrm{Z}_{2}\right.$, where $m \geq 4$.

Theorem B. Suppose that the involutions of W fall into three G-classes. Then $T$ is the direct product of two groups each of which is dibedral or semidibe. dral.

These results are best-possible in that all the $T$ named actually occur under the hypotheses. Examples are: Theorem $\mathrm{A}(\mathrm{a}), \mathrm{PSL}_{4}(q)$ for $q \equiv 3 \bmod 4$ and $\mathrm{PSU}_{4}(q)$ for $q \equiv 1 \bmod 4$; (b) $\mathrm{PSP}_{4}(q)$ for $q \equiv 3 \bmod 4$; (c) $\operatorname{PSL}_{5}(q)$ for $q \equiv$ $3 \bmod 4$ and $\mathrm{PSU}_{5}(q)$ for $q \equiv 1 \bmod 4$; Theorem $\mathrm{B}$, any direct product of two simple groups each of whose Sylow 2-subgroups is dihedral or semidihedral.

The notation used is mostly standard (and coincides with that of [12]). An. involution is an element of order 2 in some group. $D_{n}$ denotes the dihedral group of order $n$, and $E_{n}$ the elementary Abelian group of order $n . \Sigma_{n}$ and $\Sigma_{n}^{+}$denote respectively the symmetric and alternating groups of degree $n$.

If $H$ and $K$ are subgroups of some group $X$, and $K$ normalizes $H$, then $\mathbf{A}_{K}(H)$, called "the automizer of $H$ in $K$ ", is the group of automorphisms of $H$ induced by $K$ (by conjugation).

If $x$ and $y \in$ some group $X$, and $H \leq X$, then $x \sim_{H} y$, or " $x$ is fused to $y$ in $H^{\prime \prime}$, means that there is $b \in H$ with $x^{b}=y . x \sim y$ means $x \sim_{X} y$.

If $H$ is a subgroup of some group $X$, a transversal to $H$ in $X$ means a complete set of coset representatives for $H$ in $X$.

If $H \leq$ some group $X$, then $H^{\#}$ means the set of nonidentity elements of $H$.

Since $G$ has no proper 2-quotient, the transfer homomorphism $\nu: G \rightarrow T / T^{\prime}$ (or into any quotient of $T / T^{\prime}$ ) is always the trivial homomorphism. We shall frequently use the following consequence of this, of which a proof is given in [12]: 
Thompson transfer theorem. Let $T$ be a Sylow 2-subgroup of a finite group $G$ with no proper 2-quotient, and let $M$ be a maximal subgroup of $T$. Let $x$ be any involution of $G-M$. Then some G-conjugate of $x$ lies in $M$.

We shall also use other transfer arguments, which will be spelled out explicitly as they occur.

The first step in proving Theorems $A$ and $B$ is to show that we may assume $T$ has a normal subgroup $V \cong \mathrm{Z}_{4} \times \mathrm{Z}_{4}$; for then $\mathrm{C}_{T}(V)$ is a metacyclic 2-group (Alperin [1]), and this fact is a great help in constructing $T$. We shall prove

Theorem 1. Let $T$ be a Sylow 2-subgroup of a fusion-simple group G. As. sume that $T$ bas no normal elementary subgroup of order $8 ; N_{G}(T)=T C_{G}(T)$; $T \approx D_{8}$; and the involutions of the unique normal four-group $W$ of $T$ are not all G-conjugate. Then $T$ bas a normal subgroup isomorpbic to $\mathbf{Z}_{4} \times \mathbf{Z}_{4}$.

Proof. Suppose not. $W \triangleleft T$; if $W=\mathrm{C}_{T}(W)$, then $T=W$ or $T \cong D_{8}$, and both of these contradict our hypotheses, by Burnside's theorem (Huppert [11, IV. 2.5, p. 418]).

Suppose $W<B \cong \mathrm{Z}_{4} \times \mathrm{Z}_{2}$, where $B \triangleleft T$ and $B=\mathbf{C}_{T}(B)$. If $W \leq \mathbf{Z}(T)$, then $\mathbf{A}_{T}(B)$ is elementary and so every $\gamma \in T-B$ has $\gamma^{2} \in B \cap \mathrm{C}_{T}(\gamma)=W$, so $\Phi(T)=$ $W$. From $W \leq \mathrm{Z}(T)$ and $\mathbf{N}_{G}(T)=T \mathrm{C}_{G}(T)$, it follows by Burnside's theorem and the hypothesis that no two involutions of $W$ are fused in $G$. $T$ contains involutions $\gamma \notin W$ fused to the involutions of $W$, by Glauberman's $Z^{*}$-theorem [8]. But for such a $\gamma,\langle\gamma, W\rangle$ is a normal $E_{8}$ of $T$.

Hence $W$ is noncentral in $T$, and if $\langle z\rangle=\mho^{1}(B)$, then $z$ is not $G$-conjugate to any other element of $B$. Aut $(B) \simeq D_{8}$, and $A_{T}(B)$ is a subgroup of Aut $(B)$ which does not centralize $W$. We shall write $T_{0}=\mathrm{C}_{T}(W)$, of index 2 in $T$.

First, suppose $A_{T}(B)$ is cyclic of order 4 . Then all the involutions of $T$ lie in $T_{0}=\langle\zeta, B\rangle$ where $\zeta$ inverts $B$. So if $\zeta^{2} \neq 1$, then $\Omega_{1}(T)=W$, and $z$ is not conjugate to any other involution of $T$, violating the $\mathrm{Z}^{*}$-theorem. So there are $\tau, \zeta \in T$ such that $T=\langle\tau, B\rangle$ with $\tau^{2}=\zeta, \zeta^{2}=1$, and $\zeta$ inverting $B . \Phi\left(T_{0}\right)$ $=W$, and so $\langle\zeta, W\rangle \triangleleft T_{0}$; but $r$ also normalizes $\langle\zeta, W\rangle$, so $\langle\zeta, W\rangle$ is a normal $E_{8}$ of $T$.

Next suppose $A_{T}(B)$ is elementary. We claim no involution of $T-T_{0}$ is fused to $z$. For if $r$ is an involution of $T-T_{0}$, then we can take notation in $B$ so that $B=\langle a\rangle \times\langle b\rangle, a^{2}=z ; b^{\tau}=z b, a^{\tau}=a$. If $\tau \sim z$, there is $g \in G$ with $\tau^{8}=$ $z$ and $\mathrm{C}_{T}(\tau)^{8} \leq T$ (by Sylow's theorem in $\left.\mathrm{C}_{G}(z)\right)$. Then $z^{8} \in \Phi(T) \leq W$, so $z^{g}=z$, contrary to $r^{8}=z$.

$\left|A_{T}(B)\right|=2$ would give $T_{0}=B$, and hence $z$ not conjugate to any other element of $T$. Hence $\left|\mathbf{A}_{T}(B)\right|=4$, and $T_{0}=\langle\zeta, B\rangle$ where $\zeta^{2}=1, \zeta \sim z$, and $\zeta$ inverts $B$. 
Some $\sigma \in T-T_{0}$ has $a^{\sigma}=a, b^{\sigma}=z b$, and $\sigma^{2} \in\langle a\rangle=\mathrm{C}_{B}(\sigma)$. Now $T=\langle B$, $\zeta, \sigma\rangle$ and so $\zeta^{\sigma}=\zeta a w$ for some $w \in W$, otherwise $\langle\zeta, W\rangle \triangleleft T$. Hence, $\left(\sigma \zeta^{2}=\right.$ $\sigma^{2} \zeta^{\sigma} \zeta=\sigma^{2} a^{-1} w$. So $\left(\sigma \zeta^{2} \in W\right.$ if and only if $\sigma^{2} \notin W$. Replacing $\sigma$ by $\sigma \zeta$ if necessary, we get $\sigma^{2} \in\langle z\rangle=\mathrm{C}_{w}(\sigma)$. Then, replacing $\sigma$ by $\sigma b$ if necessary, we get $\sigma^{2}=1$. So notation may be chosen in $B$ with

$T=\langle B, \zeta, \tau\rangle$ where $B=\langle a\rangle \times\langle b\rangle, a^{2}=z ; \zeta^{2}=1, \zeta$ inverts $B, \tau^{2}=1, a^{\top}=$ $a$ and $b^{T}=b z, \zeta=\zeta a w$ for some $w \in W$.

Now $r$ inverts $[\tau, \zeta]=a w$, so $w=b$ or $z b$. Replacing $\zeta$ by $b \zeta$ if necessary, we may assume $\zeta^{\top}=\zeta a b$.

Since $\mathbf{N}_{G}(T)=T \mathrm{C}_{G}(T)$, Grün's theorem $[10$, Theorem 14.4.4, p. 214] implies that $\Gamma$ is generated by conjugates of $T^{\prime}=\langle a b\rangle$. We will show $T$ cannot be so generated, by showing that five of the seven nonidentity cosets of $T^{\prime}$ in $T$ cannot contain conjugates of elements of $T^{\prime}$. We take $\left\{\zeta, \zeta_{T}, \tau, b, \zeta b, \zeta \tau b, \pi b\right\}$ to represent the nonidentity cosets of $T^{\prime}$ in $T$.

Now $\zeta_{\tau} B$ consists of elements of order 8 , so $\zeta_{T} T^{\prime}$ and $\zeta_{\tau} b T^{\prime}$ do not contain conjugates of elements of $T^{\prime}$.

Suppose $x \in b\langle a b\rangle$ is $G$-conjugate to an element of $T^{\prime}$. Since $b$ and $z b \alpha_{G} z$, $x=a b$ or $a b z$, and we conclude that $a \sim \sim_{G} a b$. Now $\mathbf{C}_{T}(a)=\langle B, r) ; \mathbf{C}_{T}(a b)=$ $\langle B, r \zeta\rangle$; and $\mathrm{C}_{T}(a), \mathrm{C}_{T}(a b)$ are Sylow 2-subgroups of $\mathrm{C}_{G}(a), \mathrm{C}_{G}(a b)$ respectively (since $T$ has no central elements of order 4 ), so we should have $C_{T}(a) \cong$ $\mathrm{C}_{T}(a b)$ by Sylow's theorem; but $\mathrm{C}_{T}(a)$ has exponent 4 while $\mathrm{C}_{T}(a b)$ has exponent 8.

Suppose $x \in \tau\langle a b\rangle$ is $G$-conjugate to an element of $T^{\prime}$. $\tau\langle a b\rangle$ consists of involutions, so $x$ is an involution, and we have shown that no involution of $T-T_{0}$ can be G-conjugate to $z$.

We next consider $\zeta\langle a b\rangle$ and $\zeta b\langle a b\rangle$. Both are single conjugacy classes un$\operatorname{der} T$; we shall show that one of them must fail to contain conjugates of elements of $T^{\prime}$, i.e., conjugates of $z$. Suppose $\zeta \sim z$. Then there is $g \in G$ with $\zeta^{g}=z$ and $\mathrm{C}_{T}(\zeta)^{g} \leq T . \mathrm{C}_{T}(\zeta)=\langle W, \zeta) \cong E_{8}$, and $\mathrm{C}_{T}(\zeta), \mathrm{C}_{T}(\zeta)^{T}$ are the only two $E_{8}$ 's of $T$, hence $g$ may be chosen $\epsilon G$ with

$$
\zeta^{8}=z, \quad\langle W, \zeta\rangle^{8}=\langle W, \zeta\rangle .
$$

The $T$-classes of involutions in $\langle W, \zeta\rangle$ are

$$
\{z\} ;\{b, z b\} ;\{\zeta, \zeta z\} ;\{\zeta b, \zeta z b\} \text {. }
$$

Now if $\zeta b \chi_{G} b$, then $z=\Pi \mathrm{ccl}_{G}(b) \cap\langle W, \zeta\rangle$ is fixed by $\mathbf{N}_{G}(\langle W, \zeta\rangle)$, contrary to $\zeta^{g}=z$. Hence, $\zeta b \sim b$, and so every element of $\zeta b\langle a b\rangle$ is $G$-conjugate to $b$, so not $G$-conjugate to $z$. So $\zeta b\langle a b\rangle$ contains no conjugate of an element of $T^{\prime}$.

Thus, $\zeta \tau T^{\prime}, \zeta \tau b T^{\prime}, b T^{\prime}, \tau T^{\prime}$, and $\zeta b T^{\prime}$ (or $\zeta T^{\prime}$ ) all fail to contain conjugates 
of elements of $T^{\prime}$, in violation of Grün's theorem.

Next, suppose $A_{T}(B)=A u t(B)$, and $\rho, \zeta, \tau \in T$ have the following actions on $B$ :

$$
\begin{aligned}
& \rho: a \rightarrow a b, b \rightarrow b . \\
& \zeta: a \rightarrow a^{-1}, b \rightarrow b . \\
& \tau: a \rightarrow a, b \rightarrow a^{2} b .
\end{aligned}
$$

For any such $\rho, \zeta, \tau$, we have $[\zeta, \tau] \in W$ and $[\zeta, \rho] \epsilon W$. Namely, $\rho^{2} \epsilon$ $\mathbf{C}_{B}(\rho)=W$, so

$$
1=\left[\rho^{2}, \zeta\right]=[\rho, \zeta]^{\rho}[\rho, \zeta]
$$

and $\rho$ inverts $[\rho, \zeta$, so $[\rho, \zeta] \in W$. Also, the Jacobi identity gives

$$
\begin{aligned}
1 & \equiv\left[\rho, \zeta^{-1}, \tau\right]^{\zeta}\left[\zeta, \tau^{-1}, \rho\right]^{\tau}\left[\tau, \rho^{-1}, \zeta\right]^{\rho} \\
& \equiv 1 \cdot\left[\zeta, \tau^{-1}, \rho\right]^{\tau} \cdot[\zeta x, \zeta]^{\rho} \bmod \left\langle a^{2}\right\rangle, \text { for some } x \in B \\
& \equiv\left[\zeta, \tau^{-1}, \rho\right] \bmod \left\langle a^{2}\right\rangle .
\end{aligned}
$$

Hence $\left[\zeta, \tau^{-1}, \rho\right] \in\left\langle a^{2}\right\rangle$. Since $[B, \rho]=\langle b\rangle$, this requires $\left[\zeta, \tau^{-1}\right] \in \mathbf{C}_{B}(\rho)=W$.

Hence no $\zeta$ as above has $\zeta^{2}=1$, for if it did, $\langle\zeta, W\rangle$ would be a normal $E_{8}$ of $T$.

Now $T-T_{0}$ has no involutions $G$-conjugate to $z=a^{2}$. For if $\gamma \in T-T_{0}$ is conjugate to $z$, then $C_{B}(\gamma)=\langle a\rangle$ or $\langle a b\rangle$ and so if $g \in G$ has $\gamma^{8}=z$ and $C_{T}(\gamma)^{8}$ $\leq T$, then $z^{\mathcal{B}} \in \Phi(T) \leq\langle B, \zeta\rangle$, hence $z^{\mathcal{B}} \in W$, hence $z^{\mathcal{B}}=z$, contrary to $\gamma^{\mathcal{B}}=z$.

By the $Z^{*}$-theorem, $\rho B$ or $\rho \zeta B$ must contain a conjugate of $z$; choose notation so that $\rho B$ contains a conjugate of $z$, so that we can take $\rho$ so that $\rho^{2}=1$. But then $\rho$ inverts its commutator $[\rho, \tau]$, so $[\rho, \tau]$ and $[\rho, \zeta \in W$, and $\langle\rho, W\rangle$ is a normal $E_{8}$ of $T$.

We may now assume that $T$ has no normal $\mathrm{Z}_{4} \times \mathrm{Z}_{4}$, but $W \leq B \simeq \mathrm{Z}_{2^{n}} \times \mathrm{Z}_{2}$ where $n \geq 3, B \triangleleft T$, and $B=\mathrm{C}_{T}(B)$. Write $B=\langle a\rangle \times\langle b\rangle$ where $|a|=2^{n},|b|=2$; then $\operatorname{Aut}(B)=\langle\Sigma, \rho, \gamma\rangle$, where $\Sigma=$ Aut $(\langle a\rangle)$ (fixing b), so that $\Sigma \cong Z_{2^{n-2}} \times Z_{2}$; $\rho: a \rightarrow a b, b \rightarrow b$; and $r: a \rightarrow a, b \rightarrow z b$ where $z$ is the unique involution of $\langle a\rangle$. Then $\rho$ and $r$ centralize $\Sigma$, and $[\rho, r]=\pi$ where $\pi: a \rightarrow a z, b \rightarrow b$, so that $\pi$ is the unique involution of $\Phi(\Sigma)$ if $n \geq 4$.

We claim $A_{T}(B)$ does not contain $\pi$ (and hence $A_{T}(B)$ is elementary, since $\pi$ is the unique involution of $\Phi(\operatorname{Aut}(B)))$. Namely, suppose $x \in T$ with $a^{x}=a z$ and $b^{x}=b$. Then $\langle B, x\rangle \triangleleft T$. We may choose $x \in x B$ so that $x^{2}=1$ or $x^{2}=b$. If $x^{2}=1$, then $\langle W, x\rangle=\Omega_{1}(\langle B, x\rangle)$ is a normal $E_{8}$ of $T$. If $x^{2}=b$, then $\left\langle a^{2^{n-2}}, x\right\rangle=\Omega_{2}(\langle B, x\rangle)$ is a normal $\mathrm{Z}_{4} \times \mathrm{Z}_{4}$ of $T$. 
$A_{T}(B)$ is elementary, so $A_{T}(B) \leq \Omega_{1}(A u t(B))$. If $n=3, \Omega_{1}($ Aut $(B))=$ Aut $(B)$; if $n \geq 4$, then $\Omega_{1}($ Aut $(B))=\langle\phi,-1, \rho, \tau\rangle$, where -1 is inversion of $B$, and $\phi: a \rightarrow a^{1+2^{n-2}}, b \rightarrow b$, so $\phi^{2}=\pi$. Since $\pi \notin$ Aut $(B), A_{T}(B) \cap \Sigma=1$, $\langle-1\rangle$, or $\langle-\pi\rangle$. The set of involutions of $\Omega_{1}($ Aut $(B))-\Sigma$ is $\rho \Gamma \cup \tau \Gamma \cup \phi \rho \tau \Gamma$ (or, just $\rho \Gamma \cup \tau \Gamma$ if $n=3$ ), where $\Gamma=\langle-1, \pi\rangle$. Of these three cosets of $\Gamma$, the product of any two elements from distinct sets has order 4 , so $A_{T}(B)$ contains elements from at most one of $\rho \Gamma, \tau \Gamma$, and $\phi \rho \tau \Gamma$. It follows that $A_{T}(B)$ is elementary of order at most 4.

We will use Glauberman's $Z^{*}$-theorem to finish this case.

(i) Let $y \neq z$ be an involution of $T$ such that $z$ is a square in $\mathbf{C}_{T}(y)$. Then $y \not z$.

Proof. If $y \sim z$, then there is $g \in G$ with $y^{8}=z$ and $\mathbf{C}_{T}(y)^{8} \leq T$, so $z^{8} \epsilon$ $\Phi(T)$. As $\Phi(T) \leq B$, we have $z^{8} \in W$. Now the only $G$-conjugate of $z$ in $W$ is $z$ itself; this follows from the hypothesis that not all involutions of $W$ are $G$-conjugate, if $W \leq \mathbf{Z}(T)$; while if $W \measuredangle Z(T)$ it follows from Burnside's theorem and the assumption $\mathbf{N}_{G}(T)=T \mathbf{C}_{G}(T)$. Hence $z^{8}=z$, contrary to $y^{8}=z$.

(ii) Suppose that any two $E_{8}$ 's of $T$ that are $G$-conjugate are $T$-conjugate. Then there is no $y \in \mathbf{C}_{T}(W)-B$ with $y \sim z$ and $y \sim y x$ for all $x \in W$.

Proof. Suppose false; then there is $g \in G$ with $y^{8}=z$ and $\langle W, y\rangle=\langle W, y\rangle$. But $z=\Pi\left(\operatorname{ccl}_{G}(z) \cap\langle W, y\rangle\right)$ is stable under $\mathbf{N}_{G}(\langle W, y\rangle)$.

(iii) (Completion of argument if $W \leq \mathbf{Z}(T)$ ). Let $T_{0}=\mathbf{C}_{T}(W), a_{2}=a^{2^{n-2}}$.

We claim no involution of $T-T_{0}$ is conjugate to $z$. Namely, any involution $y$ of $T-T_{0}$ induces one of the following automorphisms of $B: \tau, \pi,-\tau$, - $\pi \tau$; (if $n \geq 4$ ) $\phi \rho \tau, \pi \phi \rho \tau,-\phi \rho \tau,-\pi \phi \rho \tau$. By conjugacy in Aut (B) (i.e., choice of basis in $B$ ), we need only consider $\tau,-\tau, \phi \rho r$, and $-\phi \rho r$. But each of these automorphisms fixes an element of $B$ (namely $a_{2}$ or $a_{2} b$ ) whose square is $z$, and this contradicts (i).

Hence $T_{0}=\langle a, b, s\rangle$, where $s^{2}=1, s \sim z$, and $s$ centralizes $b$ and sends $a$ to $a^{-1}$ or $a^{-1} z$, so that $\langle s, a\rangle$ is dihedral or semidihedral. $T=\langle B, s, t\rangle$ where $t$ induces one of the eight automorphisms listed above; by replacing $t$ with $s t$ and changing basis in $B$, we may assume $t$ induces $\tau$ or $\phi \rho \tau$ on $B$.

(iii.i) Assume that $W \not Z Z(T)$ and $\langle s, a\rangle$ is semidihedral.

If $t \in T-T_{0}$ induces $\tau$ on $B$, so that $a^{t}=a$ and $b^{t}=z b$, then we may assume $t^{2}=1$ or $a$. If $t^{2}=a$, then

$$
a^{2} z=[s, a]=\left[s, t^{2}\right]=[s, t][s, t]^{t} \text {. }
$$

Now $s$ inverts its commutator $[s, t]$, so $[s, t] \in\left\langle a^{2}, b\right\rangle$, but then $[s, t][s, t]^{t} \epsilon$ $\left\langle a^{4}\right\rangle$, contrary to the line above. Hence $t^{2}=1$, so $t$ inverts $[s, t]$ and hence $[s, t] \in\left\langle a_{2} b\right\rangle$. Also $\left[s, t a_{2} b\right]=z[s, t]$, so we may assume $[s, t]=1$ or $a_{2} b$. 
If $[s, t]=1$, then $t b$ centralizes $s$ and $(t b)^{2}=z$, contrary to (i). Hence $s^{t}=s a_{2} b$, and $s \sim s x$ for every $x \in W$. No element of $T-T_{0}$ centralizes an element of $T_{0}-B$, so all $E_{8}$ 's of $T$ lie in $T_{0}$ and so are $T$-conjugate to $\langle W, s\rangle$; this contradicts (ii).

It $t \in T-T_{0}$ induces $\phi \rho r$ on $B$, so that $n \geq 4$ and $a^{t}=a a_{2} b, b^{t}=z b$, then we may assume $t^{2}=1$. So $t$ inverts $[s, t]$ and $[s, t] \in\left\langle a_{2} b\right\rangle$. As before, we may assume $s^{t}=s a_{2} b$, and we need only show that every $E_{8}$ of $T$ lies in $T_{0}$. But if not, there are involutions in $s B$ and $t B$ that centralize each other, i.e., $(s x)^{t y}$ $=s x$ where $x \in\left\langle a^{2}, b\right\rangle$ and $y \in\left\langle a_{2} b\right\rangle$. But then

$$
s x=(s x)^{t y}=\left(s a_{2} b x^{t}\right)^{y} \equiv s a_{2} b x \bmod \langle z\rangle,
$$

a contradiction.

(iii.ii) Assume that $W \not \mathbf{Z}(T)$ and $\langle s, a\rangle$ is dihedral. Then $T_{0}$ has two classes of $E_{8}$ 's instead of one. The following variation of (ii) will be useful:

(iii.ii.i) Suppose every $E_{8}$ of $T$ lies in $T_{0}$; then $s \not s b$.

Proof. Since $s \sim z$, there is $g \in G$ with $s^{8}=z$ and $\langle W, s\rangle^{8}=\langle W, s\rangle$ or $\langle W, s a\rangle$. If $s \sim s b$, then $z=\Pi\left(c_{G}(z) \cap\langle W, s\rangle\right)$ is stable under $\mathbf{N}_{G}(\langle W, s\rangle)$, so we must have $\langle W, s\rangle^{g}=\langle W, s a\rangle$. But also, $\operatorname{ccl}_{G}(z) \cap\langle W, s a\rangle$ is forced to be $\{z\}$ $\cup W s a$, so $\{b, b z\}^{8}=\{b, b z\}$. Therefore $(s b)^{8}=s^{8} b^{8}=z b^{8} \chi z$, contrary to $s b$ $\sim s \sim z$.

Suppose now that $t \in T-T_{0}$ induces $\tau$ on $B$, so that $a^{t}=a$ and $b^{t}=z b$. We may assume $t^{2}=1$ or $a$. It $t^{2}=a$, then

$$
a^{2}=\left[s, t^{2}\right]=[s, t][s, t]^{t},
$$

and so $[s, t] \in a\left\langle a^{2}, b\right\rangle$.

Suppose $[s, t] \in a\left(a^{2}\right\rangle$. Then $a^{2}=[s, t][s, t]^{t}$ implies that $[s, t]=a$ or $a z$, and $\langle s, t\rangle$ is accordingly dihedral or semidihedral. If semidihedral, then all the involutions of the maximal subgroup $\langle s, t\rangle$ of $T$ are conjugate to $z$, so by transfer, $b \sim z$, contrary to assumption. Suppose $\langle s, t\rangle$ is dihedral. Now no element of $s B$ centralizes any element of $t B$, so every $E_{8}$ of $T$ lies in $T_{0}$, and so, by (iii.ii.i), $s \not \alpha s b$; also, as $\langle s, t\rangle$ is maximal in $T$, transfer gives $s t \not z . \Phi(T)=$ $T^{\prime}=\langle a\rangle$, and $T$ is generated by $s, t$, and $b$. Grün's theorem applies as in the previous case, and so we need only find five cosets of $\Phi(T)$ in $T$ which cannot contain $G$-conjugates of elements of $\langle a\rangle, s b \Phi(T)$ and $s t \Phi(T)$ are already known to have this property since they consist of conjugate involutions. $t \Phi(T)$ and $t b \Phi(T)$ have it, since their elements are all of order larger than $|a|$. We claim no element of $b \Phi(T)$ is conjugate to an element of $\langle a\rangle$, i.e., there are no $k$ and odd $m$ with $a^{2^{k} m} b \sim a^{2 k}$. Namely, if so, then $a^{2 k} \notin\langle z\rangle$, so $\mathrm{C}_{T}\left(a^{2^{k}}\right)=\langle B, t\rangle$ is a Sylow 2-subgroup of $\mathrm{C}_{G}\left(a^{2^{k}}\right)$. Since $B \leq \mathrm{C}_{T}\left(a^{2^{k} m} b\right)$, there is $g \in G$ with 
$\left(a^{2 k} m b\right)^{g}=a^{2^{k}}$ and $B^{g} \leq\langle B, t\rangle$, and hence $B^{g}=B$. But $T$ covers $\mathbf{N}_{G}(B) / C_{G}(B)$, and $a^{2 k} b$ is not $T$-conjugate to $a^{2 k}$.

Suppose now that $t^{2}=a$ and $[s, t] \in a b\left\langle a^{2}\right\rangle$. Then $a^{2}=[s, t][s, t]^{t}$ implies that $[s, t]=a a_{2} b$ or $a a_{2} b z$. We shall use transfer. Every element of $t B$ has order $2^{n+1}$, and every element of $t s B$ has order 8 . Hence, the transfer $\nu$ from $G$ to $T / T_{0}$ has the value

$$
\nu(t s)=\prod\left\{g t s g^{-1}: T g(t s)=T g\right\} T_{0}
$$

where $g$ runs over some transversal to $T$ in $G$, since terms like $g(t s)^{2} g^{-1}$ and $g(t s)^{4} g^{-1}$ have order $<8$ so lie in $T_{0}$. (We are using the form for $\nu(t s)$ given, for instance, in $[10$, Lemma 14.4 .1$, p. 206].) The number of $g$ in the transversal with $T g(t s)=T g$ is odd, so since $G$ has no proper 2-quotient, some $g(t s) g^{-1}$ lies in $T_{0}$, and hence in $B$ as its order is 8. Thus, $g(t s) g^{-1} \in a^{2 n-3}\left\langle a_{2}, b\right\rangle$, and so $a_{2} \sim(t s)^{2}=a_{2} b . \mathrm{C}_{T}\left(a_{2}\right)=\langle B, t\rangle, \mathbf{C}_{T}\left(a_{2} b\right)=\langle B, t s\rangle$, and so these are Sylow 2-subgroups of $\mathrm{C}_{G}\left(a_{2}\right)$ and $\mathrm{C}_{G}\left(a_{2} b\right)$ respectively, so should be isomorphic. But $\langle B, t\rangle$ has exponent $2^{n+1}$ and $\langle B, t s\rangle$ has exponent $2^{n}$.

We may therefore assume $t^{2}=1$. It follows as in (iii.i) that we may assume $[s, t]=a_{2} b$; this implies (via $a^{2 n-3}$ ) that $s \sim s b$. Also, no element of $s B$ centralizes an element of $T-T_{0}$, so every $E_{8}$ of $T$ lies in $T_{0}$. This contradicts (iii.ii.i).

Suppose now that $t \in T-T_{0}$ induces $\phi \rho r$ on $B$, so that $n \geq 4$ and $a^{t}=$ $a a_{2} b, b^{t}=z b$. As in (iii.i), we may assume $t^{2}=1$ and $[s, t]=a_{2} b$. This implies $s \sim s b$. The only $E_{8}$ 's of $T$ which do not lie in $T_{0}$ are $T$-conjugate to $\langle z, s a$, $\left.t a_{2} b\right\rangle$. There is $g \in G$ with $s^{g}=z$ and $\langle W, s\rangle^{g} \leq T_{0}$ or $\langle W, s\rangle^{8}=\left\langle z, s a, t a_{2} b\right\rangle$. But $\left\langle z, s a, t a_{2} b\right\rangle$ contains four elements of $T-T_{0}$; none of these are $G$-conjugate to $z$, since they centralize $a_{2}$ or $a_{2} b$ and (i) applies. $\langle W, s\rangle^{8}$ contains five conjugates of $z$. Hence $\langle W, s\rangle^{g} \leq T_{0}$, and the argument of (iii.ii.i) applies.

(iv) (Completion of argument if $W \leq \mathrm{Z}(T)$ ). Assume $W$ is central in $T$; then $\mathrm{A}_{T}(B) \leq\{1,-1,-\pi\} \cup \rho \Gamma$.

Suppose some $x \in T$ induces $\rho$ (or equivalently, $\pi \rho$ ) on $B$, so that $a^{x}=$ $a b, b^{x}=b$. We may assume $x^{2}=1$ or $b . U=\left\langle x, \mathbf{C}_{B}(x)\right\rangle=\left\langle x, a^{2}, b\right\rangle$ is a normal Abelian subgroup of $T$; and if $x^{2}=1$ then $\Omega_{1}(U) \cong E_{8}$, if $x^{2}=b$ then $\Omega_{2}(U) \simeq$ $\mathrm{Z}_{4} \times \mathrm{Z}_{4}$, so $T$ has a normal $E_{8}$ or a normal $\mathbf{Z}_{4} \times \mathbf{Z}_{4}$, contrary to assumption.

Hence $A_{T}(B)$ can contain only the elements $-\rho$ or $-\pi \rho$ of $\rho \Gamma$; and it cannot contain both, since then it would contain $\pi$. So, with suitable choice of basis, $\mathbf{A}_{T}(B)=\langle-1\rangle ;\langle-\pi\rangle$; or $\langle-\rho\rangle$.

No two involutions of $W$ are $G$-conjugate (Burnside's theorem and $N_{G}(T)=$ $T \mathrm{C}_{G}(T)$ ). By transfer, every involution of $T$ is $G$-conjugate to some involution 
of $W$, so $G$ has exactly three classes of involutions; by the $Z^{*}$-theorem, $T-B$ contains conjugates of all three involutions of $W$. In particular, $T=\langle B, x\rangle$ where $x \sim_{G} z$. If $A_{T}(B)=\langle-\pi\rangle$ or $\langle-\rho\rangle$, then the $T$-classes of involutions in $T-B$ are $x\left\langle a^{2}\right\rangle$ and $x b\left\langle a^{2}\right\rangle$, so some involution of $W$ is conjugate to no other element of $T$, violating the $\mathrm{Z}^{*}$-theorem.

Suppose $A_{T}(B)=\langle-1\rangle$. Then the $T$-classes of involutions in $T-B$ are $x\left\langle a^{2}\right\rangle, x b\left\langle a^{2}\right\rangle, x a\left\langle a^{2}\right\rangle$, and $x a b\left\langle a^{2}\right\rangle$. Precisely two of these four $T$-classes are fused in $G$.

If $x \sim x b, x a$, or $x a b$, then by replacing $a$ with $a b$ if necessary we may assume $x \sim x b$ or $x a$. If $x \sim x b$, let $x^{8}=z$ and $\langle W, x\rangle^{g} \leq T$. $\langle W, x a\rangle$ contains at most three $G$-conjugates of any of its involutions, while $\langle W, x\rangle$ contains five conjugates of $z$, so we may assume $\langle W, x\rangle^{8}=\langle W, x\rangle$. But $z=\operatorname{\Pi ccl}_{G}(z) \cap\langle W, x\rangle$ is stable under $\mathrm{N}_{G}(\langle W, x\rangle)$. If $x \sim x a$, then the maximal subgroup $\langle a, x\rangle$ of $T$ has only one $G$-class of involutions, so by transfer, $G$ has only one class of involutions, which is false.

Hence $x$ is not fused to any of $x b, x a$, or $x a b$. If $x b \sim x a b$ or $x a$, we can choose notation in $B$ so that $x b \sim x a$; then the maximal subgroup $\langle a, x b\rangle$ of $T$ has only two $G$-classes of involutions, so by transfer, so does $G$, which is false.

Hence $x a \sim x a b$. Choose $b \in\{b, z b\}$ so that $x a \sim b$. Then there is $g \in G$ such that $(x a)^{g}=b$ and $\langle W, x a\rangle^{g} \leq T$. $\langle W, x a\rangle$ contains five $G$-conjugates of $b$, while $\langle W, x\rangle$ contains at most three $G$-conjugates of any of its involutions. Hence, we may assume $\langle W, x a\rangle^{g}=\langle W, x a\rangle$. But $b=\prod_{c c l}(b) \cap\langle W, x a\rangle$ is stable under $\mathbf{N}_{G}(\langle W, x a\rangle)$.

Now any normal $A$ belian subgroup $B$ of $T$ with $W \leq B$ and $B=C_{T}(B)$ must have rank 2 , and since $T$ has no normal $Z_{4} \times Z_{4}, B \cong Z_{2 n} \times Z_{2}$ for some $n \geq 1$; we have discussed all cases $(n=1, n=2, n \geq 3)$ and shown none can occur. Thus the proof of Theorem 1 is complete.

In view of Theorem 1, we may now assume that $T$ contains a normal subgroup $V \cong \mathrm{Z}_{4} \times \mathrm{Z}_{4^{\circ}}, W=\Omega_{1}(V)$ is the unique normal four-group of $T . \mathbf{C}_{T}(V)$ is metacyclic (Alperin [1]), so $\Omega_{1}\left(C_{T}(V)\right)=W$.

The argument will divide according to fusion patterns in elementary subgroups of $\mathbf{C}_{T}(W)$, containing conjugates of central involutions of $T$.

Lemma 1. $\mathrm{C}_{T}(W)-\mathrm{C}_{T}(V)$ contains a conjugate of some central involution of $T$.

Proof. If $W \leq \mathbf{Z}(T)$ this is just the $\mathbf{Z}^{*}$-theorem. If $W \notin \mathbf{Z}(T)$, the $\mathbf{Z}^{*}$-theorem tells us that $T-\overrightarrow{\mathbf{C}}_{T}(V)$ contains a conjugate $\gamma$ of the unique central involution $\omega$ of $T$. If $\gamma \notin \mathrm{C}_{T}(W)$, then there are $a, b \in V$ such that $\gamma$ exchanges $a$ and $b$, and $\gamma$ centralizes $a b$, and $(a b)^{2}=\omega$. By Sylow's theorem in $C_{G}(\omega)$, there is $g$ $\epsilon G$ with $\gamma^{g}=\omega$ and $(a b)^{g} \in T$; then $\omega^{g} \in \Phi(T) \leq C_{T}(W)$, but $\omega^{g} \neq \omega$. The other 
two involutions of $W$ are not conjugate to $\omega$, so $\omega^{8} \notin \mathbf{C}_{T}(V)$; hence $\omega^{8} \in \mathbf{C}_{T}(W)$ $-\mathbf{C}_{T}(V)$, as desired.

Lemma 2. Every $E_{16}$ of $T$ lies in $\mathrm{C}_{T}(W)$.

Proof. We may choose a basis for $V$ such that $A_{T}(V) \leq\left\langle B^{+},\left(\begin{array}{ll}0 & 1 \\ 1 & 0\end{array}\right)\right\rangle$, where $B^{+}$denotes the subgroup of Aut $(V)$ which fixes $W$ elementwise.

Suppose $E$ is an $E_{16}$ of $T$ with $E \notin C_{T}(W)$. Then $|E \cap W|=2$, and in Aut $(V),\left(\begin{array}{ll}0 & 1 \\ 1 & 0\end{array}\right)$ must centralize each automorphism induced on $V$ by $E \cap \mathbf{C}_{T}(W)$. Now $\mathbf{C}_{\boldsymbol{O}_{+}}\left(\left(\begin{array}{ll}0 & 1 \\ 1 & 0\end{array}\right)\right)$ has order 4 ; so $E=\langle\omega, z, y, t\rangle$ where $z$ inverts $V, y$ has matrix $\left(\begin{array}{ll}1 & 2 \\ 2 & 1\end{array}\right)$ on $V$, and $t$ has matrix $\left(\begin{array}{ll}0 & 1 \\ 1 & 0\end{array}\right)$ on $V$.

We claim that if $\Omega_{3}\left(C_{T}(V)\right)$ has order 64 , then $\left(\begin{array}{ll}1 & 2 \\ 2 & 1\end{array}\right)$ cannot be induced by an involution on $V$. Namely, let $\{a, b\}$ be the chosen basis for $V$; then there is a basis $\{c, d\}$ for $\Omega_{3}\left(\mathbf{C}_{T}(V)\right)$ such that $c^{2}=a, d^{2}=b$, and $[c, d]=1$ or $\omega$. If

$$
c^{y}=c^{a} d^{\beta}, d^{y}=c^{\gamma} d^{\delta},
$$

then $\beta$ and $\gamma$ are even because $y$ centralizes $W$ and so centralizes $\Omega_{3}\left(C_{T}(V)\right) / V$; and then

$$
\begin{aligned}
& a^{y}=\left(c^{2}\right)^{y}=\left(c^{\alpha} d^{\beta}\right)^{2}=c^{2 a} d^{2 \beta}=a b^{2}=c^{2} d^{4}, \\
& b^{y}=c^{2 \gamma} d^{2 \delta}=a^{2} b=c^{4} d^{2} ;
\end{aligned}
$$

hence $\alpha \equiv \delta \equiv 1 \bmod 4$, while $\beta \equiv \gamma \equiv 2 \bmod 4$. And

$$
c=c^{y^{2}}=c^{a^{2}+\beta y} d^{\beta(a+\delta)} \text {; }
$$

but $a+\delta \equiv 2 \bmod 4$, while $\beta \equiv 2 \bmod 4$, so $\beta(\alpha+\delta) \equiv 4 \bmod 8$, so our equation is impossible.

Hence $\Omega_{3}\left(C_{T}(V)\right)$ has order $\leq 32$, so that some element of $V$ lies outside $\Phi\left(\mathrm{C}_{T}(V)\right)$; since $V$ is central in $\mathrm{C}_{T}(V)$ and $\mathrm{C}_{T}(V)$ is a 2 -generator group, we get that $\mathbf{C}_{T}(V)$ is Abelian. Therefore, the set $S$ of elements of $\mathbf{C}_{T}(V)$ inverted by $z y$ is a subgroup of $C_{T}(V)$. But $\Omega_{2}(S)=S \cap V=W$; hence $S=W$.

Now for any $x \in \mathrm{C}_{T}(W),[x, z y] \in \mathrm{C}_{T}(V)$ and is inverted by the involution $z y$; so $\left[\mathbf{C}_{T}(W), z y\right] \leq W$. Also $t$ normalizes $\langle W, z y\rangle$. So $T=\left\langle\mathbf{C}_{T}(W), t\right\rangle_{\text {normal- }}$ izes $\langle W, z y\rangle$, contrary to the assumption that $T$ has no normal $E_{8}$.

We shall want to study $E_{16}$ 's of $T$. The following remarks about $E_{16}$ 's will be useful:

Lemma 3. Let $F$ be any $E_{16}$ of $G$.

(i) Let $T^{*}$ be any Sylow 2-subgroup of $G$ that contains $F$; let $V^{*}$ be a normal $\mathbf{Z}_{4} \times \mathbf{Z}_{4}$ of $T^{*}$. Then $V^{*}$ and $F$ normalize eacb otber, and $\mathbf{C}_{F}\left(V^{*}\right)=$ $W^{*} \leq F$. 
(ii) Suppose $F$ contains more than one G-conjugate of some central involution $\omega$ of $T$. Then no conjugate $\gamma$ of $\omega$ with $\gamma \in F$ can be central in $\mathbf{N}_{G}(F)$. (This implies that $\mathbf{A}_{G}(F)$ is not a 2-group.)

(iii) Suppose $F$ contains more than one G-conjugate of some central involution $\omega$ of $T$. Suppose $F$ is not a Sylow 2-subgroup of $\mathrm{C}_{G}(F)$. Let $T^{*} \geq K>F$ where $T^{*}, K$ are Sylow 2.subgroups of $G, C_{G}(F)$ respectively. Then $\Phi(K) \cap F$ is of order 2, and $\Phi(K) \cap F=\langle\xi\rangle$ where $\xi \in W^{*}, \xi \chi \omega$, and $\xi$ is central in $\mathbf{N}_{G}(F)$.

Proof. (i) By Lemma 2, $F$ centralizes $W^{*}$, and so by the four-generator theorem of [12], $F \geq W^{*}:$ Also, $\left[V^{*}, F\right] \leq W^{*}$, so $V^{*}$ and $F$ normalize each other. Alperin's theorem (Alperin [1]) in $T^{*}$ implies that $\mathbf{C}_{F}\left(V^{*}\right)=W^{*}$.

(ii) Suppose $\gamma \in F$ is conjugate to $\omega$ and is central in $\mathbf{N}_{G}(F)$. Let $\delta \in F$, $\delta \sim \omega$, and $\delta \neq \gamma$. Let $T^{*} \geq F$ where $T^{*}$ is a Sylow 2-subgroup of $\mathbf{C}_{G}(\delta)$ (and hence of $G$ ). Then by (i), $\mathrm{C}_{F}\left(V^{*}\right)=W^{*}$, so $W^{*}=\langle\delta, \gamma\rangle$. But $\delta$ is the only. conjugate of $\delta$ to lie in $W^{*}$ (by Burnside's theorem and the hypotheses on $N_{G}(T)$ and $W$ ).

(iii) $K>F$ implies $\Phi(K) \neq 1$, by the four-generator theorem. $K<\mathbf{C}_{T^{*}}\left(W^{*}\right)$, so the involutions of $\Phi(K)$ all centralize $V^{*}$, so lie in $W^{*}$. So $1<\Phi(K) \cap F$ $\leq W^{*}$.

Let $N=\mathbf{N}_{G}(F)$; then $N=\mathbf{C}_{G}(F) \mathbf{N}_{N}(K)$ (Frattini argument), so $N$ normalizes $\Phi(K) \cap F$. If $\Phi(K) \cap F=W^{*}$ or $\left\langle\omega^{*}\right\rangle$ (where $\omega^{*} \sim_{G} \omega$ ), then $\omega^{*}$ would be central in $N$, contrary to (ii). Hence, (iii) holds.

Next, we have some useful results concerning 2 -groups.

Lemma 4. There is no 2-group $T$ with $V \triangleleft T, V \cong \mathbf{Z}_{4} \times \mathbf{Z}_{4}, \mathbf{C}_{T}(V)=V$, $T$ baving no normal $E_{8}$, and $\left[\mathrm{C}_{T}\left(\Omega_{1}(V)\right): V\right]=16$.

Proof. Let $W=\Omega_{1}(V)$, and let $T_{0}=\mathrm{C}_{T}(W)$. If $\left[T_{0}: V\right]=16, T_{0}$ induces on $V$ the full subgroup $B^{+}$(as in the proof of Lemma 2) of Aut $(V)$.

There is a four-group of $B^{+}$of which every involution $a$ has $C_{V}(a)=W$. (Namely, the matrices 1, $\left(\begin{array}{ll}3 & 0 \\ 2 & 3\end{array}\right),\left(\begin{array}{ll}3 & 2 \\ 0 & 3\end{array}\right),\left(\begin{array}{ll}1 & 2 \\ 2 & 1\end{array}\right)$ form such a four-group; this fourgroup can also be described as $\left[B^{+}, \phi\right]$ where $\phi$ is any automorphism of order 3 in Aut $(V)$.) Let $R / V$ induce this four-group on $V$. Then every $r \in R$ has $r^{2} \in \mathbf{C}_{V}(r)=W$; hence $\Phi(R)=W$, and $[R: \Phi(R)]=16$.

There is $\beta \in B^{+}$such that $C_{V}(\beta)=W$ and all the elements of $V$ inverted by $\beta$ lie in $W$. (Namely, $\left(\begin{array}{ll}1 & 2 \\ 2 & 3\end{array}\right)$ ) ) Let $x \in T_{0}$ induce $\beta$ on $V$. Then $x^{2} \in \mathrm{C}_{V}(x)$ $=W$. Hence $T_{0}$ centralizes $x^{2}$, so for any $r \in R$,

$$
1=\left[x^{2}, r\right]=[x, r]^{x}[x, r] \text {. }
$$


Hence $[x, r] \in V$ and is inverted by $x$, so $[x, r] \in W$. Therefore $[R, x] \leq W$. Hence $\Phi(\langle x, R\rangle)=W$, and $\langle x, R\rangle$ is a five-generator group. But then $T$ has a normal elementary subgroup of order 8 , by the four-generator theorem of [12].

Lemma 5 (Thompson). Let $S$ be a 2-group, $A$ a normal Abelian subgroup of $S$ maximal subject to baving exponent $\leq 4$. Assume $W=\Omega_{1}(A)$ is central in $S$. For any involution $x \in S-W$, define $W(x)=\left\{z \in W: x \sim_{S} x z\right\}$. Then if $x$ and $y$ are involutions of $S-W$ with $W(x) \cap W(y)=1, x$ centralizes $y$.

Proof. $S$ stabilizes the chain $A \geq W>1$ (i.e., centralizes each of the factors $A / W$ and $W / 1)$, so $\Phi(S)$ centralizes $A$. Hence if $|x y| \geq 4$, then the involution $z$ of $\langle x y\rangle$ centralizes $A$, so lies in $A$ by Alperin's theorem [1]. But $x \sim x z$ and $y \sim y z$, so $z \in W(x) \cap W(y)$.

We shall want to apply Lemma 5 with $A=V$ and $S=\mathrm{C}_{T}(W)$. To justify this, we give

Lemma 5a. Let $T$ be a 2-group containing no normal $E_{8}$, and suppose $V \triangleleft$ $T$ where $V \simeq \mathrm{Z}_{4} \times \mathrm{Z}_{4}$. Assume $\mathrm{C}_{T}\left(\Omega_{1}(V)\right)$ bas index 2 in $T$. Then $V$ is a normal Abelian subgroup of $\mathrm{C}_{T}(W)$ and is maximal sucb subject to baving exponent $\leq 4$.

Proof. Suppose not, and let $X>V,[X: V]=2$, where $X$ is a normal Abelian subgroup of $\mathbf{C}_{T}(W)$ with exponent 4. Then $X=\langle x, V\rangle$ for some involution $x$. $\Omega_{1}(X) \cong E_{8}$, so $X \not T$ and there is $t \in T-C_{T}(W)$ with $x^{t}=y$ where $y \equiv x \bmod V$.

Let $s=x y$. Then $s \in \mathrm{C}_{T}(V)$, so $\Omega_{2}(\langle s\rangle) \leq V$, by Alperin's theorem applied to $T$ and $V$. So if $|s| \geq 4$ and $s_{2}$ is an element of order 4 in $\langle s\rangle$, then $s_{2} \in V$ and $x$ centralizes $s_{2}$; but $x$ inverts all powers of $s$. Hence $|s| \leq 2$, and $s \in V$ by Alperin's theorem, contrary to $x \not y$ mod $V$.

We shall need some remarks on metacyclic 2-groups containing a central $\mathrm{Z}_{4}$ $\times \mathbf{Z}_{4}$ :

Lemma 6. Let $L$ be a metacyclic 2-group containing a central $\mathrm{Z}_{4} \times \mathrm{Z}_{4}, V$ say (so $V=\Omega_{2}(L)$ ). Then

(a) Any section of exponent 4 in $L$ is Abelian.

(b) Let $V_{i}=\Omega_{i}(L)=\left\langle x \in L:|x| \leq 2^{i}\right\rangle$. Then every element of $V_{i}$ bas order $\leq 2^{i}$, so that $V_{i}=\left\{x \in L:|x| \leq 2^{i}\right\}$.

(c) Let $x, y \in L$; then $\left|\left[x, y^{2}\right]\right|=1 / 2|[x, y]|$ (unless $[x, y]=1$ ).

(d) Let $x, y \in L$ with $|x|=2^{m},|y|=2^{k}<2^{m}$; then $|x y|=2^{m}$.

(e) There is $r$ with $\left[V_{i}: V_{i-1}\right]=4$ for $1 \leq i \leq r$, while $\left[V_{i}: V_{i-1}\right]=2$ for $r+1 \leq i \leq \log _{2}$ (exponent $\left.(L)\right)$.

(f) If $\left[V_{i}: V_{i-1}\right]=4$, then the map $x \rightarrow x^{2^{i-1}}$ induces an (operator)-isomorphism of $V_{i} / V_{i-1}$ onto $W$. 
(8) If $x, y \in L$ and $x^{2}=y^{2}$, then $x \equiv y \bmod W$.

Proof. (a) Let $M / S$ be a section of exponent 4 in $L$. Then $M$ is metacyclic, so is generated by $x, y$ with $\langle x\rangle \triangleleft M$ and $y^{-1} x y=x^{a}$ for some number $a$. Since $\Omega_{2}(\langle x\rangle)$ is central in $L, a \equiv 1 \bmod 4$. Hence $M^{\prime}=\left\langle x^{a-1}\right\rangle \leq M^{4} \leq S$ since $M / S$ has exponent 4 .

(b) We need to show that if $x, y \in L$ have order $\leq 2^{i}$, so does $x y$. Suppose this is false, and let $i$ be the smallest integer for which it is false (then $i \geq 3$ ). Let $D=\left\langle x \in L:|x| \leq 2^{i-1}\right\rangle=\left\{x \in L:|x| \leq 2^{i-1}\right\}$. Let $x$ and $y$ have orders $\leq 2^{i}$ but $|x y| \geq 2^{i+1}$.

If $|x|<|y|=2^{i}$, then, in $L / D, x=1$ and so $x y=y ; y^{2} \in D$ implies $(x y)^{2}$ $\epsilon D$, so that $\left|(x y)^{2}\right| \leq 2^{i-1}$, so $|x y| \leq 2^{i}$.

If $|x|=|y|=2^{i}$, then, in $L / D, x$ and $y$ are involutions; if $|x y| \geq 4$ in $L / D$ then $L / D$ contains a $D_{8}$, contrary to (a).

(c) We may assume $[x, y] \neq 1$. Let $\langle z\rangle$ be a normal cyclic subgroup of $L$ which contains $L^{\prime}$; then

$$
\begin{aligned}
{[x, y] } & =z^{2^{k} i} \text { for some } k \text { and some odd } i \\
{\left[x, y^{2}\right] } & =[x, y][x, y]^{y}=z^{2^{k}}\left(z^{a}\right)^{2^{k}} i
\end{aligned}
$$

but $a \equiv 1 \bmod 4$, so

$$
\left[x, y^{2}\right]=z^{2^{k+1} j} \text { for some odd } j
$$

and (c) follows.

(d) $(x y)^{2}=x y x y=x x y[y, x] y=x^{2} y d y$, where $d=[y, x]$. Let $M=\langle x, y\rangle$. Now $M^{2}=\Phi(M)=\left\langle x^{2}, y^{2}\right\rangle$ is generated by elements of order $\leq 2^{m-1}$; herice every element of $\Phi(M)$ has order $\leq 2^{m-1}$. Also, $M^{4}=\left\langle x^{4}, y^{\frac{4}{4}}\right\rangle$ and every element of $M^{4}$ has order $\leq 2^{m-2}$. $M / M^{4}$ has exponent 4 , so is Abelian by (a). Hence $d \in M^{4}$ and so has order $\leq 2^{m-2}$.

$(x y)^{2}=x^{2} y^{2} d^{y} .\left|y^{2}\right|,\left|d^{y}\right| \leq 2^{m-2}$, so $\left|y^{2} d^{y}\right|<2^{m-2}$, while $\left|x^{2}\right|=2^{m-1}$. The lemma follows by induction.

(e) Suppose not; then, for some $i,\left[V_{i}: V_{i-1}\right]=2$ but $\left[V_{i+1}: V_{i}\right]=4$. Then $V_{i+1} / V_{i-1}$ has order 8 , exponent 4 (hence is Abelian), and only one involution, which is impossible.

(f) It suffices to show that $x \rightarrow x^{2}$ induces an isomorphism of $V_{i} / V_{i-1}$ onto $V_{i-1} / V_{i-2}$, and iterate. $V_{i-1}=\Phi\left(V_{i}\right)=V_{i}^{2}$, so the induced map is onto. For $x, y \in V_{i}$,

$$
(x y)^{2}=x y x y=x^{2} y[y, x] y .
$$


Now $V_{i} / V_{i}^{4}$ is Abelian, and $V_{i}^{4}=V_{i-2}$, so $[y, x] \in V_{i-2}$. Hence $(x y)^{2} \equiv$ $x^{2} y^{2} \bmod V_{i-2}$, so $x \rightarrow x^{2}$ induces an isomorphism.

(g) Let $x=y t$. Then $x^{2}=(y t)^{2}=y^{2} t^{y} t$, so $y$ inverts $t$. Since $V=\Omega_{2}(L)$ is central in $L$, this implies $t^{2}=1$.

We will now assume that the involutions of $W$ fall into two $G$-classes, say $\{\omega\}$ and $\left\{\omega_{1}, \omega_{2}\right\}$.

Theorem A. Let $T$ be a Sylow 2-subgroup of a finite group $G$ which is fusion-simple. Assume that $T$ bas no normal elementary subgroup of order 8; $\mathbf{N}_{G}(T)=T \mathrm{C}_{G}(T) ; T \nRightarrow D_{8} ;$ and the involutions of the unique normal four-group $W$ of $T$ fall into two G-classes.

Then one of the following bolds:

(a) $T \simeq D_{2 m} \backslash \mathbf{Z}_{2}$, where $m \geq 4$.

(b) $T=\left\langle\langle\alpha, \lambda\rangle \times\langle\beta, \mu\rangle, \pi, \tau: a^{2}=\lambda^{2^{n}}=\beta^{2}=\mu^{2^{n}}=1, a \lambda \alpha=\lambda^{-1}, \beta \mu \beta=\right.$ $\mu^{-1} ; \pi^{2}=\lambda \mu, \pi$ centralizes $\lambda$ and $\mu,[\alpha, \pi]=\lambda,[\beta, \pi]=\mu ; \tau^{2}=1, \alpha^{\tau}=\beta$, $\left.\lambda^{\top}=\mu, \pi^{\top}=\pi\right\rangle$, where $n \geq 2$.

(c) $\left.T \simeq D_{2 m}^{+}\right\} \mathbf{Z}_{2}$, where $m \geq 4$.

Proof of Theorem A. Firstly, if $W \leq \mathbf{Z}(T)$, then the two G-conjugate involutions $\omega_{1}, \omega_{2}$ of $W$ must be conjugate in $N_{G}(T)$, by Burnside's theorem. But this is impossible because $\mathbf{N}_{G}(T)=T C_{G}(T)$. Hence $W \not Z Z(T)$, and $\Omega_{1}(Z(T))=$ $\langle\omega\rangle$ say has order 2 ; and $\omega_{1}, \omega_{2} \in W-Z(T)$.

Let $V$ be a normal $\mathbf{Z}_{4} \times \mathbf{Z}_{4}$ of $T$ ( $V$ exists by Theorem 1). By Lemma 1, $\mathbf{C}_{T}(W)-\mathbf{C}_{T}(V)$ cor:ains a conjugate $\alpha$ of $\omega$. By Alperin's theorem [1], a does not centralize $V$, so there is $v \in V$ with $a^{v}=a x$, where $x \in W-\{1\}$. Then if $y \in W-\langle x\rangle$, we have $(a y)^{v}=a x y$. Hence, for all $\alpha \sim \omega, \alpha \in \mathbf{C}_{T}(W)-\mathbf{C}_{T}(V)$, we have $\left|\operatorname{ccl}_{G}(\alpha) \cap a W\right|=2$ or 4 .

Hence, we can partition the proof of Theorem $A$ into three cases:

1. $\mathbf{C}_{T}(W)-\mathbf{C}_{T}(V)$ contains $a \sim \omega$ with every element of $a W$ conjugate to $\omega$.

2. 1 is false, but $\mathrm{C}_{T}(W)-\mathrm{C}_{T}(V)$ contains $a \sim \omega$ with $\operatorname{ccl}_{G}(\omega) \cap a W=$ $\left\{a, a \omega_{1}\right\}$.

3. 1 and 2 are false, but $\mathrm{C}_{T}(W)-\mathrm{C}_{T}(V)$ contains $a \sim \omega$, and, for every such $a, \operatorname{ccl}_{G}(\omega) \cap \alpha W=\{a, a \omega\}$.

We shall write $T_{0}$ for $\mathrm{C}_{T}(W)$.

Case 1. There is $a \sim \omega$ in $T_{0}-\mathrm{C}_{T}(V)$ with $a W \leq \operatorname{ccl}_{G}(\omega)$. Let $T^{*}$ be a Sylow 2-subgroup of $\mathrm{C}_{G}(a)$, and hence of $G$, containing $\langle a, w\rangle . W^{*} \cap\langle a, w\rangle$ contains $a$. If it contained more, it would contain a conjugate of $\omega_{1}$, so $W^{*}=$ $\left\langle a, \omega_{1}\right\rangle$ or $\left\langle a, \omega_{2}\right\rangle$; but these both contain two conjugates of $\omega$, while $W^{*}$ contains only one conjugate of $\omega$.

Hence $W^{*} \cap W=1$. Since $W \leq T^{*},\left[W^{*}, W\right] \leq\langle a\rangle$, so $W^{*}$ normalizes $\langle a, W\rangle$. 
Now $\omega_{1}$ and $\omega_{2}$ are the only members of their $G$-conjugacy class in $\langle a, W\rangle$, so are fixed or exchanged by $W^{*}$. If they are exchanged, then $\omega_{1} \omega_{2}=\omega \in\left[W, W^{*}\right]$, which is false. Hence $\left[W^{*}, W\right]=1$, and $F=W^{*} W=W^{*} \times W \cong E_{16}$.

Let $\left(W^{*}\right)^{\#}=\left\{a, a_{1}, a_{2}\right\}$. Then $\omega_{1}, \omega_{2}, a_{1}, a_{2} \in F$ are $G$-conjugate to $\omega_{1}$, while $a, \omega, a \omega, a \omega_{1}, a \omega_{2} \in F$ are $G$-conjugate to $\omega$. We shall now determine the full $G$-fusion pattern in $F$, and show that $A_{G}(F)$ contains a subgroup isomorphic to $\Sigma_{3} \times \Sigma_{3^{\prime}}$

$F \leq T_{0}^{*}$ (Lemma 2), so, for each $f \in F-W^{*}, 1 \neq\left[f, V^{*}\right] \leq W^{*}$ (where $V^{*}$ is any normal $\mathbf{Z}_{4} \times \mathbf{Z}_{4}$ of $\left.T^{*}\right)$. In particular, $\omega_{1} \sim \omega_{1} x$ for some $x \in\left(W^{*}\right)^{\#}$ and $\omega_{2} \sim \omega_{2} y$ for some $y \in\left(W^{*}\right)^{\#}$. Therefore $\left|\operatorname{ccl}_{G}\left(\omega_{1}\right) \cap F\right| \geq 6$.

If 5 divides $\left|A_{G}(F)\right|$, then $F$ must contain 10 conjugates of $\omega_{1}$ and 5 of $\omega$, and the product of the 5 conjugates of $\omega$ is 1 . But $a \cdot \omega \cdot a \omega \cdot a \omega_{1} \cdot a \omega_{2}$ $=\omega \neq 1$.

If 7 divides $\left|\mathbf{A}_{G}(F)\right|$, then there is a hyperplane of $F$ whose involutions are all $G$-conjugate, and $F$ has at most one additional involution of this conjugacy class. Hence, there is a three-member subset $S$ of $\left\{\omega_{1}, \omega_{2}, a_{1}, a_{2}\right\}$, or a four-member subset $S$ of $\left\{\alpha, \omega, \alpha \omega, \alpha \omega_{1}, \alpha \omega_{2}\right\}$, such that all involutions of $\langle S\rangle$ are conjugate. But this is false.

Hence $\mathbf{A}_{G}(F)$ is a $\{2,3\}$-group, and is not a 2-group by Lemma 3(ii).

We now note that $\mathbf{A}_{G}(F)$ has no fixed points on $F^{\#}$; for if $N=\mathbf{N}_{G}(F)$, then $\mathbf{C}_{F}(N) \leq W^{*} \cap W=1$, by Lemma 3(ii).

The size of each orbit of $N$ on $F$ is a $\{2,3\}$-number, and so $F$ contains at least 6 conjugates of $\omega$. But if $F$ contained exactly 6 conjugates of $\omega$, their product would be fixed by $N$ and so must be 1 , which means that the sixth would be the product of the five we have already; but this product is $\omega$, which would not be a sixth element. Hence $\left|\operatorname{ccl}_{G}(\omega) \cap F\right| \geq 7$.

Suppose $\left|\mathrm{ccl}_{G}(\omega) \cap F\right|=7$. Then there is some union of orbits of $N$ which has size 7. Hence $N$ contains no fixed-point-free 3-element, and the 3-elements of $N$ have orbits as follows:

$$
\{x\} ;\{y\} ;\{x y\} ;\{a, b, a b\}=Y^{\#} \text { say; } x Y^{\#} ; y Y^{\#} ; x y Y^{\#} \text {. }
$$

Each orbit of $N$ is a union of these, and it follows that $\mathrm{ccl}_{G}(\omega) \cap F$ falls into an orbit of $N$ with size 4 and one with size 3 . Since $N$ has no fixed points on $F$, the product of the elements in each orbit is 1 . Hence the orbits are $Y^{\#}$ and (say) $\{x\} \cup x Y^{\#}$. In particular, $\operatorname{ccl}_{G}(\omega) \cap F$ is a hyperplane of $F$. But we have already seen that this is false; and indeed, the same argument shows that $\left|\mathrm{ccl}_{G}\left(\omega_{1}\right) \cap F\right| \neq 7$.

Suppose $\left|\mathrm{ccl}_{G}(\omega) \cap F\right|=8$. Then $\left|\mathrm{ccl}_{G}\left(\omega_{1}\right) \cap F\right|=6$ or 7.7 is impossible as we have just shown; and 6 would leave one element for $F^{\#}-\operatorname{ccl}_{G}(\omega)$ $\cup \operatorname{ccl}_{G}\left(\omega_{1}\right)$, necessarily central in $N$. 
Hence $\left|\operatorname{ccl}{ }_{G}(\omega) \cap F\right|=9$ and $\left|\operatorname{ccl}_{G}\left(\omega_{1}\right) \cap F\right|=6$. This means that there is precisely one $x \in\left(W^{*}\right)^{\#}$ with $\omega_{1} \sim \omega_{1} x$, and precisely one $y \in\left(W^{*}\right)^{\#}$ with $\omega_{2} \sim$ $\omega_{2} y$. By interchanging $\alpha_{1}$ and $\alpha_{2}$ if necessary, we may assume

$$
\omega_{1} \sim \omega_{1} \alpha_{1}
$$

(Note that $\omega_{1} \sim \omega_{1} \alpha_{1}$ or $\omega_{2} \alpha_{2}$, not $\omega_{1} \alpha$ since $\omega_{1} \alpha \sim \omega_{\text {. }}$ ) Then the product of all the conjugates of $\omega_{1}$ lying in $F$ must be 1 , and so

$$
\omega_{2} \sim \omega_{2} a_{2}
$$

Hence, $F=\left\langle\omega_{1}, a_{1}\right\rangle \times\left\langle\omega_{2}, a_{2}\right\rangle$ where $\left\langle\omega_{1}, a_{1}\right\rangle$ and $\left\langle\omega_{2}, a_{2}\right\rangle$ contain all the conjugates of $\omega_{1}$, and all the "crosselements" are conjugate to $\omega_{\text {. }}$. The automorphism-group of this pattern in $F$ is isomorphic to $\Sigma_{3} \backslash Z_{2}$. We will now show that $A_{G}(F)$ contains a subgroup isomorphic to $\Sigma_{3} \times \Sigma_{3}$, where one direct factor acts on $\left\langle\omega_{1}, \alpha_{1}\right\rangle$ and the other acts on $\left\langle\omega_{2}, \alpha_{2}\right\rangle$.

Let $A=\mathbf{A}_{G}(F)$. $A$ is not a 2-group; $\Sigma_{3}\left\{\mathbf{Z}_{2}\right.$ is 3 -closed, so $\mathbf{O}_{3}(A)>1$. If $\mathbf{0}_{3}(A)$ were generated by a single 3 -element whose centralizer $C$ in $F$ were a four-group, then $A$ would leave $C$ invariant, and since $A / \mathbf{0}_{3}(A)$ is a 2-group, $A$ would have a fixed point on $F$. Hence $\mathrm{O}_{3}(A)$ contains a fixed-point-free 3 element.

Since $A$ has no fixed points on $F, F$ is a Sylow 2-subgroup of $\mathbf{C}_{G}(F)$ by Lemma 3(iii), and so $V$ induces a four-group on $F$. Hence $A$ contains a fourgroup, and we must show that $A$ cannot have as Sylow 2-subgroup the four-group $\langle\gamma, \delta\rangle$ where $\gamma$ exchanges $\left\langle\omega_{1}, \alpha_{1}\right\rangle$ and $\left\langle\omega_{2}, a_{2}\right\rangle$ and $\delta$ acts nontrivially on each. Namely, if it did, then $\langle\gamma, \delta\rangle$ would be induced by $V$; but $[F,\langle\gamma, \delta\rangle]$ contains an involution from each of $\left\langle\omega_{1}, \alpha_{1}\right\rangle$ and $\left\langle\omega_{2}, \alpha_{2}\right\rangle$, and also a four-group consisting of elements in neither one ("crosselements"), so $[F,\langle\gamma, \delta\rangle]$ has order 8; but $[F, V] \leq W$ which has order 4 .

Hence, the four-group induced by $V$ is $\left\langle\delta_{1}, \delta_{2}\right\rangle$ where $\delta_{1}$ centralizes $\left\langle\omega_{1}, a_{1}\right\rangle$ and $\delta_{2}$ centralizes $\left\langle\omega_{2}, a_{2}\right\rangle$. Now $\left\langle\delta_{1}, \delta_{2}\right\rangle$ does not normalize any cyclic group generated by a fixed-point free 3 -element. Hence $\left|\mathbf{O}_{3}(A)\right|=9$, and $A$ contains $\Sigma_{3} \times \Sigma_{3}$, as claimed.

(i) $\mathrm{C}_{T}(V)>V_{10}$

Proof. Suppose false. $\left[T: T_{0}\right]=2$, and $\left[T_{0}: V\right] \leq 8$ by Lemma 4 . $F$ induces a four-group on $V$, so $4 \leq\left[T_{0}: V\right] \leq 8$.

We first find the exact isomorphism type of $F V$, which is as follows: there are $x, y \in V$ such that $V=\langle x, y\rangle$ and $F V=\left\langle x, y, a_{1}, a_{2}\right\rangle$ where $x^{2}=\omega_{1}, y^{2}=$ $\omega_{2}, a_{1}$ inverts $x$ and centralizes $y$, and $\alpha_{2}$ inverts $y$ and centralizes $x_{0}$. To prove this, we first note that the fusion pattern of involutions of $F$ implies that $\left[V, a_{1}\right]=\left\langle\omega_{1}\right\rangle$ and $\left[V, a_{2}\right]=\left\langle\omega_{2}\right\rangle$, so that $\mathbf{C}_{V}\left(a_{1}\right)$ and $\mathbf{C}_{V}\left(a_{2}\right)$ both have order 8. Let 


$$
\mathbf{C}_{V}\left(a_{1}\right)=\langle w, y\rangle ; \quad \mathbf{C}_{V}\left(a_{2}\right)=\langle W, x\rangle .
$$

We have that $F$ is a Sylow 2-subgroup of $\mathbf{C}_{G}(F)$ (Lemma 3(iii)), so $x \neq y \bmod W$, and $\left[a_{1}, x\right]=\omega_{1},\left[a_{2}, y\right]=\omega_{2^{\prime}}$. It remains to show $x^{2}=\omega_{1}$ and $y^{2}=\omega_{2^{\prime}}$. Now $x$ centralizes $\left\langle a_{2}, \omega_{2}\right\rangle=F_{2}$ say, and acts nontrivially on $\left\langle a_{1}, \omega_{1}\right\rangle=F_{1} ; y$ centralizes $F_{1}$ and acts nontrivially on $F_{2}$. There is a 3 element $\delta$ of $\mathbf{N}_{G}(F)$ which acts nontrivially on $F_{2}$ and centralizes $F_{1}$, and so centralizes $x \bmod \mathrm{C}_{G}(F)$. Thus $x$ normalizes $\left\langle\delta, \mathrm{C}_{G}(F)\right\rangle . \mathrm{C}_{G}(F)=F \times R$ where $R$ has odd order, and the subgroups of order $3|R|$ in $\left\langle\delta, \mathrm{C}_{G}(F)\right\rangle$ are $\langle\delta c, R\rangle$ where $c \in F$ satisfies $c^{\delta^{2}} c^{\delta} c$ $=1$, so $c \in F_{2}$. Hence

$$
\begin{aligned}
x^{\delta} & =x c z \quad \text { where } z \in R, c \in F_{2} ; \\
\left(x^{2}\right)^{\delta} & =(x c z)^{2}=x^{2} c^{x} c z^{x} z .
\end{aligned}
$$

But $x^{2} \in F$, so $\left(x^{2}\right)^{\delta} \in F$ and $z^{x} z=1$. Also, $c^{x} c=1$ because $c \in F_{2}$. Hence $\left(x^{2}\right)^{\delta}=x^{2}$, so $x^{2} \in W^{\#} \cap F_{1}^{\#}=\left\{\omega_{1}\right\}$. Similarly, $y^{2}=\omega_{2}$.

Now take $\{x, y\}$ as a basis for $V \cdot x^{2} y^{2}=\omega_{1} \omega_{2}=\omega$ is the unique central involution of $T$, so with respect to this basis, the matrix-group induced by $T$ on $V$ is contained in the Sylow 2-subgroup $\left\langle B^{+},\left(\begin{array}{ll}0 & 1 \\ 1 & 0\end{array}\right)\right\rangle$ of Aut $(V)$. The matrix-group induced by $F$ is $\left\langle\left(\begin{array}{ll}3 & 0 \\ 0 & 1\end{array}\right),\left(\begin{array}{ll}1 & 0 \\ 0 & 3\end{array}\right)\right\rangle$, which is normalized by $\left(\begin{array}{ll}0 & 1 \\ 1 & 0\end{array}\right)$ and $B^{+}$. Hence, $F V \triangleleft T$.

Take $\phi \in T-T_{0}$, and let $U=\mathrm{A}_{T_{0}}(V)$. The action of $\phi$ on $U$ can be determined from the action of $\left(\begin{array}{ll}0 & 1 \\ 1 & 0\end{array}\right)$ on $B^{+}$. Namely, if $T_{0}=F V$, then $\langle U, \phi\rangle=D_{8}$ and we can choose $\phi$ so that $\phi^{2}$ centralizes $V$. If $\left[T_{0}: V\right]=8$, we have

$$
\mathrm{C}_{U}(\phi) \geq[U, \phi], \quad\left|\mathrm{C}_{U}(\phi)\right||[U, \phi]|=8,
$$

and so $\left|\mathrm{C}_{U}(\phi)\right|=4$ and $\mathrm{C}_{U}(\phi)=\left\langle\left(\begin{array}{ll}3 & 0 \\ 0 & 3\end{array}\right),\left(\begin{array}{ll}1 & 2 \\ 2 & 1\end{array}\right)\right\rangle=\mathrm{C}_{\boldsymbol{\theta}+}(\phi)$. Hence $\phi$ can be chosen so that $\phi^{2}$ induces 1 or $\left(\begin{array}{ll}1 & 2 \\ 2 & 1\end{array}\right)$ on $V$.

Now $\left\langle a_{1}, V\right\rangle$ contains precisely two $E_{8}$ 's, namely $\left\langle a_{1}, w\right\rangle$ and $\left\langle a_{1}, x, w\right\rangle$. $V$ normalizes them both. Suppose $c \in T_{0}$ induces $\left(\begin{array}{ll}1 & 2 \\ 2 & 1\end{array}\right)$ on $V$; then we claim $c$ also normalizes both these $E_{8}$ 's. Namely, $c^{2} \in \mathbf{C}_{V}(c)=W$ implies that $c$ inverts $\left[a_{1}, c\right]$, so $\left[a_{1}, c\right] \in\langle x y, w\rangle$; but $a_{1}^{2}=1$ implies that $a_{1}$ inverts $\left[a_{1}, c\right]$, so $\left[a_{1}, c\right] \epsilon\langle x, W\rangle$; hence $\left[a_{1}, c\right] \in W$, so $c$ normalizes both $E_{8}$ 's. Similarly, $c$ normalizes $\left\langle a_{2}, W\right\rangle$ and $\left\langle\alpha_{2} y, W\right\rangle$. .

Let $E=\left\langle a_{1}, W\right\rangle$. Now $\phi^{2}$ normalizes $E$ (whether $\phi^{2}$ induces 1 or $\left(\begin{array}{ll}1 & 2 \\ 2 & 1\end{array}\right)$ on $V$ ), so $\phi$ normalizes $\left\langle E, E^{\phi}\right\rangle$. ( $E^{\phi}$ is $\left\langle\alpha_{2}, W\right\rangle$ or $\left.\left\langle\alpha_{2} y, W\right\rangle_{0}\right) E$ and $E^{\phi}$ are normalized by $c$ and $F V$, so by $T_{0}$. Hence $\left\langle E, E^{\phi}\right\rangle \triangleleft T$.

But, by Lemma 5a, $V$ is maximal in $T_{0}$ subject to being Abelian of exponent 44. Hence Lemma 5 applies to $a_{1}$ and $a_{1}^{\phi}$, giving that $a_{1}^{\phi}$ centralizes $a_{1}$. Hence $\left\langle E, E^{\phi}\right\rangle$ is a normal $E_{16}$ of $T$, contrary to hypothesis on $T$. 
This finishes the proof of (i).

$\mathbf{C}_{T}(V)$ is a metacyclic group (Alperin [1]). We now employ a construction which will give a homocyclic Abelian subgroup of index 1,2 , or 4 in $\mathbf{C}_{T}(V)$.

For $\gamma$ any involution of $T_{0}-W$, and $H$ any subgroup of $G$, we define

$$
W(\gamma ; H)=\left\{v \in W: \gamma \sim_{H} \gamma v\right\} \text {. }
$$

Further, define

$$
\begin{aligned}
& I\left(\omega_{1}\right)=\left\{\gamma \in T_{0}-W: \gamma \sim \omega_{1} \text { and } W(\gamma ; T)=\left\langle\omega_{1}\right)\right\}, \\
& I\left(\omega_{2}\right)=\left\{\gamma \in T_{0}-W: \gamma \sim \omega_{1} \text { and } W(\gamma ; T)=\left\langle\omega_{2}\right)\right\} .
\end{aligned}
$$

Then $a_{1} \in I\left(\omega_{1}\right), a_{2} \in I\left(\omega_{2}\right)$. By Alperin's theorem, if $\gamma \in I\left(\omega_{j}\right)$,

$$
1<W(y ; V) \leq W\left(\gamma ; T_{0}\right) \leq W(\gamma ; T)=\left\langle\omega_{j}\right\rangle,
$$

for $j=1,2$; and nence $W\left(\gamma ; T_{0}\right)=\left\langle\omega_{j}\right\rangle$, so that $I\left(\omega_{1}\right)$ and $I\left(\omega_{2}\right)$ centralize each other elementwise (by application of Lemmas $5 \mathrm{a}$ and 5 to $V$ and $T_{0}$ ). Hence

$$
H_{1}=\left\langle I\left(\omega_{1}\right)\right\rangle \quad \text { and } \quad H_{2}=\left\langle I\left(\omega_{2}\right)\right\rangle
$$

centralize each other.

(ii) $H_{1}=\left\langle I\left(\omega_{1}\right)\right\rangle$ has $\lambda_{0} \in V \cap H_{1}$ with $\lambda_{0}^{2}=\omega_{1}$.

Proof. Let $\phi$ be any element of $T-T_{0}$, and let $z=\phi^{-1} \alpha_{1} \phi$. Then $W(z ; T)$ $=\phi^{-1} W\left(a_{1} ; T\right) \phi=\phi^{-1}\left\langle\omega_{1}\right) \phi=\left\langle\omega_{2}\right\rangle$, and so $\left[\alpha_{1}, z\right]=1$ by Lemma 5. Hence $E=$ $\left\langle a_{1}, z, w\right\rangle \cong E_{16}$.

$\left[V, a_{1}\right]$ and $[V, z]$ are of order 2 , so $C_{V}\left(a_{1}\right)$ and $C_{V}(z)$ are of order 8. We claim $\mathbf{C}_{V}\left(\alpha_{1}\right) \neq \mathbf{C}_{V}(z)$. Namely, $E$ contains more than one conjugate of $\omega$ (e.g., $\omega$ and $\left.\alpha_{1} \omega\right)$, so Lemma 3 (iii) applies to $E$. Hence, if $\mathbf{C}_{V}\left(\alpha_{1}\right)=\mathbf{C}_{V}(z)$, then $E$ would not be a Sylow 2-subgroup of $\mathbf{C}_{G}(E)$, and so $\omega_{1}$ or $\omega_{2}$ would be central in $\mathbf{N}_{G}(E)$. If $\omega_{1}$ is central in $\mathbf{N}_{G}(E)$, let $T^{*}>T_{0}^{*}>E$ where $T^{*}$ and $T_{0}^{*}$ are Sylow 2-subgroups of $G$ and $\mathbf{C}_{G}\left(\omega_{1}\right)$ respectively. $W^{*} \leq E$ and $\mathbf{C}_{E}\left(V^{*}\right)=W^{*}$, by Lemma 3(i). Hence $W^{*}=\left\langle\omega_{1}, a_{1}\right\rangle$, which is impossible since $\left\langle\omega_{1}, a_{1}\right\rangle$ contains no conjugate of $\omega$. We may assume $\omega_{2}$ is central in $\mathbf{N}_{G}(E)$. Now there is $\delta \in G$ which cycles $\alpha_{1}, \omega_{1}$, and $\alpha_{1} \omega_{1} ; \phi^{-1} \delta \phi$ then cycles $z, \omega_{2}$, and $z \omega_{2}$, so $z \omega_{2} \not \omega$. Let $T^{*}>T_{0}^{*}>E$ where $T^{*}$ and $T_{0}^{*}$ are Sylow 2-subgroups of $G$ and $\mathrm{C}_{G}(z)$ respectively. Then, as above, $W^{*}=\left\langle z, \omega_{2}\right\rangle$ which is impossible.

Let $\mathbf{C}_{V}\left(a_{1}\right)=\langle a, W\rangle, \mathbf{C}_{V}(z)=\langle b, w\rangle$. Then $c=a b$ has order 4 , and $c^{2}=$ $\omega$ since $\phi$ exchanges $\mathbf{C}_{V}\left(a_{1}\right)$ and $\mathbf{C}_{V}(z)$ so that $\langle c, W\rangle<T$.

Let $U=\Omega_{3}\left(\mathrm{C}_{T}(V)\right)$. Then $U>V$, by (i). The map $u \rightarrow u^{4}(u \in U)$ induces a homomorphism from $U / V$ into $W$, and this homomorphism commutes with T-conjugation. So if $[U: V]=2$, the image of this homomorphism is $\langle\omega\rangle$; so, whether $[U: V]=2$ or 4 , there is $y \in U$ with $y^{2}=c$. Also, $T_{0}$ centralizes $U, V$, so that $a_{1}^{y}=a_{1} v$ for some $v \in V$. Then 


$$
a_{1} \omega_{1}=a_{1}^{c}=\alpha_{1}^{y^{2}}=\left(a_{1} v\right)^{y}=\alpha_{1} v^{2}
$$

since $y$ centralizes $V$. Hence $v^{2}=\omega_{1}, v \in V$, and $v=a_{1} a_{1}^{y} \in H_{1}$. This proves (ii).

(iii) Write $H=\left\langle H_{1}, \omega\right\rangle$, and take $\lambda_{0} \in H_{1} \cap V$ with $\lambda_{0}^{2}=\omega_{1}$. Then there is $A \triangleleft H$ with $\mathrm{C}_{H}(A)=A,\left\langle\lambda_{0}, W\right\rangle \leq A$, and $A \cong \mathrm{Z}_{2 r} \times \mathrm{Z}_{2}$ for some $r \geq 2$. The involutions of $I\left(\omega_{1}\right)$ are all congruent $\bmod A$, so that $[H: A]=2$.

Proof. We first show that there is no $K \triangleleft T_{0}, K \leq H$, with $K \cong \mathbf{Z}_{\mathbf{4}} \times \mathbf{Z}_{\mathbf{4}}$. For if so, let $\phi \in T-T_{0}$; then $K^{\phi} \leq H^{\phi}$, so $K^{\phi}$ centralizes $K$, and $\left\langle K, K^{\phi}\right\rangle$ is a normal Abelian subgroup of $T$ of exponent 4. Hence $\left\langle K, K^{\phi}\right\rangle$ has order 16 , so $K=K^{\phi}$, i.e., $K \triangleleft T$. Then $\Omega_{1}(K)=W$, since $T$ has only one normal four-group. $H^{\phi}$ centralizes $H$ and hence $K$, so the involutions of $H^{\phi}$ lie in $\Omega_{1}\left(C_{T}(K)\right)=W$ (Alperin [1]); but $a_{2} \in H^{\phi}, a_{2} \notin W$.

Now $B=\left\langle\lambda_{0}, W\right\rangle \leq H$, and $B \triangleleft T_{0}$. So there is a normal Abelian subgroup $A$ of $H$ with $B \leq A$ and $A=\mathrm{C}_{H}(A)$; and we may take $A$ to be normal in $T_{0}$. Then $A$ has rank 2 , since $\left\langle A, A^{\phi}\right\rangle$ is a normal Abelian subgroup of $T$ (where $\phi \in T$ $\left.T_{0}\right)$ and so has rank 2. $\Omega_{2}(A)=B$.

We claim that $A=\mathrm{C}_{H}(B)$. For if not, there is $R$ with $A<R \leq \mathrm{C}_{H}(B),[R: A]$ $=2$, and $R \triangleleft T_{0}$. Now $A>B$ (for if not, $A=\mathrm{C}_{H}(A)=\mathrm{C}_{H}(B)$ ); let $A=\langle\lambda\rangle \times\langle\omega\rangle$, $|\lambda|=2^{n} \geq 8$. Then $R=\langle A, r\rangle$ where $r^{2} \in A . r^{2}$ centralizes $\lambda$ and $r$ centralizes $\Omega_{2}(\langle\lambda\rangle)$, so $\lambda^{r}=\lambda z$ for some $z \in W-\{1\}$. $r^{2} \in \mathbf{C}_{A}(r)=\mathbf{Z}(R)=\left\langle\lambda^{2}, \omega\right\rangle$. The coboundary group of $r$ on $A$ is $\left\langle\lambda^{2} z\right\rangle$, so we may take $r^{2} \in W$. Now $[R: Z(R)]=4$, and the three maximal subgroups of $R$ which contain $\mathbf{Z}(R)$ are $A ;\langle r, \mathbf{Z}(R)\rangle$; and $\langle r \lambda, \mathbf{Z}(R)\rangle$. $A$ and $\langle r \lambda, Z(R)\rangle$ have exponent $2^{n}$, while $S=\langle r, Z(R)\rangle$ has exponent $2^{n-1}$. Hence $S \triangleleft T_{0}$. If $r^{2}$ can be taken as 1 , then $K=\Omega_{1}(S)$ is elementary of order 8 , and for any $\phi \in T-T_{0}, K^{\phi} \leq H^{\phi}$ which centralizes $H$, so $\left\langle K, K^{\phi}\right\rangle$ is a normal elementary subgroup of $T$ of rank $\geq 3$. If $r^{2}$ cannot be taken as 1 , then $K=\Omega_{2}(S) \cong$ $\mathbf{Z}_{4} \times \mathbf{Z}_{4}$, and $K \triangleleft T_{0}$, which we have already shown is impossible.

Now let $\gamma \in I\left(\omega_{1}\right) . \gamma \notin A$ since $\Omega_{1}(A)=W$, so $\gamma$ induces a nontrivial automorphism of $B$, i.e., $\lambda_{0}^{\gamma}=\lambda_{0} z$ for some $z \in W-\{1\}$. But then $\gamma \sim \gamma z$, so by definition of $I\left(\omega_{1}\right), z=\omega_{1}$. Hence, all elements of $I\left(\omega_{1}\right)$ are congruent $\bmod \mathbf{C}_{H}(B)$ $=A$.

(iv) Let $H$ and $A$ be as in (iii). Then there is $\lambda \in A$ such that $A=\langle\lambda\rangle \times\langle\omega\rangle$, and $\alpha_{1}$ inverts $\lambda ;|\lambda|=2^{n} \geq 4 ; \alpha_{1}\left\langle\lambda^{2}\right\rangle$ and $a \lambda\left\langle\lambda^{2}\right\rangle \leq I\left(\omega_{1}\right)$, while $\alpha_{1} \omega\left\langle\lambda^{2}\right\rangle$ does not meet $I\left(\omega_{1}\right)$. Thus, $H>H_{1}$ if and only if $\alpha_{1} \omega \lambda \notin I\left(\omega_{1}\right)$.

Proof. There is $\pi \in A$ with $A=\langle\pi\rangle \times\langle\omega\rangle$, and $|\pi|=2^{n} \geq 4$. Then there is $\gamma \in I\left(\omega_{1}\right)$ with $a_{1} \gamma \in A-\left\langle\pi^{2}, \omega\right\rangle$, for if not, $H=\left\langle I\left(\omega_{1}\right), \omega\right\rangle \leq\left\langle\alpha_{1}, \pi^{2}, \omega\right\rangle\langle H$, Take $\lambda=a_{1} \gamma$ for some such $\gamma$. Clearly $\alpha_{1}\left\langle\lambda^{2}\right\rangle$ and $\alpha_{1} \lambda\left\langle\lambda^{2}\right\rangle \leq I\left(\omega_{1}\right) \cdot a_{1} \omega \cdot$ $I\left(\omega_{1}\right)$ because we know from the fusion pattern in $F$ that $a_{1} \omega \sim \omega$. 
For $\phi \in T-T_{0}$, we now let

$$
D=\left\langle H, H^{\phi}\right\rangle=\left\langle\alpha_{1}, \lambda\right\rangle \times\left\langle\alpha_{2}, \mu\right\rangle
$$

(where $\mu$ is to $H^{\phi}$ as $\lambda$ is to $H$ ). Note that if $T_{0}=D$, the isomorphism type of $T$ is determined.

(v) Suppose $\left.T_{0}\right\rangle D=\left\langle\alpha_{1}, \lambda\right\rangle \times\left\langle\alpha_{2}, \mu\right\rangle$. Then

(a) Each element of $T_{0} / D$ acts semiregularly on the set of $D$-classes of $E_{16}$ 's of $D$ (and hence $\left[T_{0}: D\right] \leq 4$ ).

(b) $H_{1}=\left\langle\alpha_{1}, \lambda\right\rangle$; hence $\langle\lambda\rangle,\langle\mu\rangle \triangleleft T_{0}$ and are exchanged by $T / T_{0}$, and $T_{0}$ $=F \mathrm{C}_{T}(\mathrm{~V})$.

Proof. (a) Let $x \in T_{0}-D$ fix a class of $E_{16}$ 's of $D$; we may assume $x$ normalizes $E=\left\langle\gamma_{1}, \gamma_{2}, W\right\rangle$ where $\gamma_{1} \in I\left(\omega_{1}\right), \gamma_{2} \in I\left(\omega_{2}\right)$. Now from the definition of $I\left(\omega_{1}\right)$ and $I\left(\omega_{2}\right)$, all automorphisms of $E$ that can be induced by an element of $T_{0}$ are already induced by $V$. So, adjusting $x$ from $V$ if necessary, we may assume $x$ centralizes $E$. $x$ normalizes $H=\left\langle\gamma_{1}, \lambda, \omega\right\rangle$, so normalizes the unique $\mathrm{Z}_{2^{n}} \times \mathrm{Z}_{2}$ of $H$. Hence $\lambda^{x} \equiv \lambda^{a} \bmod w$, for some odd integer $a$. Then

$$
\begin{aligned}
& \left(\gamma_{1} \lambda\right)^{x}=\gamma_{1}^{x} \lambda^{x} \equiv \gamma_{1} \lambda^{a} \equiv\left(\gamma_{1} \lambda\right) \lambda^{a-1} \bmod W ; \\
& \left(\gamma_{2} \mu\right)^{x} \equiv\left(\gamma_{2} \mu\right) \mu^{b-1} \bmod W,
\end{aligned}
$$

for some odd $b$. $a-1$ and $b-1$ are even, and so $x$ fixes every class of $E_{16}$ 's in $D$. So, adjusting $x$ again from $D$, we may assume $x$ normalizes $F$. But $\mathbf{N}_{T_{0}}(F)=F V \leq D$, so $x \in D$.

(b) Let $x D \in \mathrm{Z}\left(T_{0} / D\right)$. Then $x$ must move the classes of $E_{8}$ 's in both $H$ and $H^{\phi}$. Adjusting $x$ from $D$, we may assume $\alpha_{1}^{x} \equiv \alpha_{1} \lambda \bmod W$. Adjusting further from $V$, we may assume $\alpha_{1}^{x}=\alpha_{1} \lambda$ or $\alpha_{1} \lambda \omega$. If the latter, then

$$
\left(\alpha_{1} \omega\right)^{x}=\alpha_{1} \lambda \omega \omega=\alpha_{1} \lambda \sim \alpha_{1} .
$$

But we know from the fusion pattern in $F$ that $\alpha_{1} \omega \sim \omega_{1}$. Hence $\alpha_{1}^{x}=\alpha_{1} \lambda$, $\left(\alpha_{1} \omega\right)^{x}=\alpha_{1} \lambda \omega$, so $\alpha_{1} \lambda \omega \notin I\left(\omega_{1}\right)$, hence $H>H_{1}=\left\langle\alpha_{1}, \lambda\right\rangle$ where $\lambda$ is as described in (iv).

We shall write

$$
\Delta=\langle\lambda, \mu\rangle ; \quad C=\mathbf{C}_{T}(V) .
$$

The structure of $C$ now follows:

(vi) If $T_{0}=D$, then $C=\Delta$.

If $\left[T_{0}: D\right]=2$, then $C$ is Abelian and $\Phi(C)=\left\langle\lambda \mu, \Delta^{2}\right\rangle_{\text {. }}$

If $\left[T_{0}: D\right]=4$, then $\Phi(C)=\Delta$, and $C=\langle\rho, \sigma\rangle$ where $\rho^{2^{n}}=\omega_{1}, \sigma^{2^{n}}=\omega_{2}$, and $[\rho, \sigma]=1$ or $\omega$. $\left(\right.$ Here $2^{n}=|\lambda|=|\mu|$.) 
Proof. The map $x \rightarrow x^{2^{n-1}}$ induces an isomorphism of $\Delta / \Delta^{2}$ onto $W$, and this isomorphism commutes with $T$-conjugation; so $T_{0}$ centralizes $\Delta / \Delta^{2}$.

Suppose $\left[T_{0}: D\right]=2$. Then $C=\langle\pi, \Delta\rangle$, where $\pi^{2} \in \Delta . C / \Delta$ is metacyclic and Abelian, so $\pi^{2} \notin \Delta^{2} ; \pi^{2} \Delta^{2}$ is $T$-invariant in $\Delta / \Delta^{2}$, so $\pi^{2}=\lambda \mu \bmod \Delta^{2}$.. $\pi$ normalizes $\langle\lambda\rangle$ and $\langle\mu\rangle$, and centralizes $V$; so, since $T / T_{0}$ exchanges $\langle\lambda\rangle$ and $\langle\mu\rangle$, there is $j=0$ or 1 with $\lambda^{\pi}=\lambda \omega_{1}^{j}$ and $\mu^{\pi}=\mu \omega_{2}^{j}$. Then $\pi$ centralizes $\Delta^{2}$, and $(\lambda \mu)^{\pi}=\lambda \mu \omega^{j}$. But $\pi$ centralizes $\pi^{2}$, so $\pi$ centralizes $\left\langle\lambda \mu, \Delta^{2}\right\rangle$, and $j=0$ as claimed.

Suppose $\left[T_{0}: D\right]=4$. Then $C / \Delta^{2}$ has order 16 , has a central four-group $\Delta / \Delta^{2}$, and is metacyclic. Therefore, $C / \Delta^{2}$ has exponent 4. By Lemma 6(a), $C / \Delta^{2}$ is Abelian with $\Delta / \Delta^{2}$ as Frattini subgroup, so that $C$ is generated by elements $\rho, \sigma$ with $\rho^{2} \equiv \lambda$ and $\sigma^{2} \equiv \mu \bmod \Delta^{2} . \rho$ and $\sigma$ centralize $V$ and normalize $\langle\lambda\rangle$ and $\langle\mu\rangle$; so there are $i=0$ or 1 and $j=0$ or 1 with $\lambda^{\rho}=\lambda \omega_{1}^{i}, \mu^{\sigma}=\mu \omega_{2}^{i}, \lambda^{\sigma}$ $=\lambda \omega_{1}^{j}$, and $\mu^{\rho}=\mu \omega_{2}^{j}$. Then $C=\langle\rho, \sigma\rangle$ centralizes $\Delta^{2}$. Since $\rho^{2} \in \lambda \Delta^{2}$ and $\rho$ centralizes $\rho^{2}$, we have $i=0$. Then $(\lambda \mu)^{\rho \sigma}=\lambda \mu \omega_{\%}^{j}$. But $(\rho \sigma)^{2} \in \lambda \mu \Delta^{2}$ and $\rho \sigma$ centralizes $(\rho \sigma)^{2}$, so $j=0$. So $C^{2}=\Delta$ is central in $C ; C^{\prime}=\langle[\rho, \sigma]\rangle$; and $[\rho, \sigma]^{2}$ $=1$, so $[\rho, \sigma]=1$ or $\omega$, as claimed.

(vii) If $\left[T_{0}: D\right]=2$, then for any $\pi \in C_{T}(V)-\Delta$ with $\pi^{2}=\lambda \mu$, we have

$$
\left[a_{1}, \pi\right]=\lambda \omega_{1}^{\xi}, \quad\left[a_{2}, \pi\right]=\mu \omega_{2}^{\xi},
$$

where $\xi=0$ or 1 .

Proof. $\left[a_{1}, \pi\right]^{2}=\left[a_{1}, \pi^{2}\right]=\left[a_{1}, \lambda \mu\right]=\lambda^{2}$; hence $\left[a_{1}, \pi\right]=\lambda w$ for some $w \epsilon$ W. $w \in H_{1}$, so $w \in\left\langle\omega_{1}\right\rangle$, and so $\left[a_{1}, \pi\right]=\lambda \omega_{1}^{\xi}$ where $\xi=0$ or 1 . Let $\phi \in T-$ $T_{0} ; \alpha_{1}^{\phi} \in \alpha_{2} \Delta$, so $\alpha_{1}^{\phi}$ and $\alpha_{2}$ have the same effect on $\pi$ by conjugation, so $\left[\alpha_{2}, \pi\right]=\mu \omega_{2}^{\xi}$.

(viii) $T-T_{0}$ contains an involution.

Proof. (viii) holds if $T / D-T_{0} / D$ contains an involution $\delta D$. For then there is a normal series of $D$ whose factors are four-groups upon which $\delta$ acts nontrivially, and $\delta^{2} \in D$; it follows that $\delta D$ contains an involution.

Hence we may assume $\left[T_{0}: D\right]=2$ and $T / D$ is cyclic of order 4 (for if $\left[T_{0}\right.$ : $D]=4$, then $\left.T / D \cong D_{8}\right)$. Let $\delta \in T-T_{0}$; then $\delta$ interchanges $a_{1} C$ and $a_{2} C$, so $\delta^{2} \epsilon\left\langle a_{1} \alpha_{2}, C\right\rangle$; by replacing $\delta$ with $\delta \alpha_{1}$ if necessary, we may assume $\delta^{2} \epsilon$ $C$. Hence $\delta^{2} \in \pi \Delta$, and centralizes $\Delta$. So we may choose $\lambda$ and $\mu \in\langle\lambda\rangle$ and $\langle\mu\rangle$ so that $\lambda^{\delta}=\mu, \mu^{\delta}=\lambda$. Then $\mathrm{C}_{\Delta}(\delta)=\langle\lambda \mu\rangle$. Further, $\left(\delta \lambda^{i}\right)^{2}=\delta^{2} \lambda^{i} \mu^{i} ;\left(\delta \lambda^{i}\right)^{4}=$ $\delta^{4}\left(\lambda^{2 i} \mu^{2 i}\right)$. As $\delta^{4} \in \mathrm{C}_{\Delta}(\delta)-\Delta^{2}, \delta^{4}$ is an odd power of $\lambda \mu$, so we can replace $\delta$ by an appropriate $\delta \lambda^{i}$ to get

$$
\delta^{2}=\pi, \quad \pi^{2}=\lambda \mu ; \quad\left[\alpha_{1}, \pi\right]=\lambda \omega_{1}^{\xi}, \quad\left[\alpha_{2}, \pi\right]=\mu \omega_{2}^{\xi},
$$

by (vii). 
We next find the elements of $T$ whose fourth powers or squares are $\omega$. They are

$\epsilon C:$ Order 8: If $n \geq 3$, we get $\lambda_{1} \mu_{1} V$, where $\lambda_{1}=\lambda^{2^{n-3}}$ and $\mu_{1}=\mu^{2^{n-3}}$. If $n=2$, we get $\pi V$. Order 4: $\lambda_{0} \mu_{0} W$, where $\mu_{0}=\lambda^{2^{n-2}}$ and $\mu_{0}=\mu^{2^{n-2}}$.

$\epsilon \delta C \cup \delta a_{1} a_{2} C:$ None, since in $T / \Delta, \delta^{2} \equiv \pi$.

$\epsilon a_{1} C \cup a_{2} C$ : None, since $\left(a_{1} \Delta\right)^{2}=\left\langle\mu^{2}\right\rangle$ and $\left(a_{1} \pi \Delta\right)^{2}=\mu \omega_{1}^{\xi}\left\langle\mu^{2}\right\rangle$.

$\epsilon a_{1} a_{2} C:\left(a_{1} a_{2} \Delta\right)^{2}=1,\left(a_{1} a_{2} \pi \Delta\right)^{2}=\omega^{\xi}$; so the only occurrence is that if $\xi=1$, we have $\left(a_{1} a_{2} \pi \Delta\right)^{2}=\omega$.

$\epsilon \delta a_{1} C \cup \delta a_{2} C:$ For $y \in C$,

$$
\left(\delta \alpha_{1} y\right)^{2} \equiv\left(\delta \alpha_{1}\right)^{2} \bmod \Delta \equiv \delta^{2} \alpha_{1}^{\delta} \alpha_{1} \equiv \pi \alpha_{1} \alpha_{2} .
$$

And $\left(\pi \alpha_{1} \alpha_{2} \Delta\right)^{2}=\omega^{\xi}$. So if $\xi=0, \delta \alpha_{1} C \cup \delta \alpha_{2} C$ consists of elements of order 4 whose squares $\epsilon \pi a_{1} a_{2} \Delta$; if $\xi=1$, then $\delta a_{1} C \cup \delta a_{2} C$ consists of elements of order 8 whose fourth powers are $\omega$.

Now consider the transfer $\nu: G \rightarrow T / T_{0}$. Using the form given in $[10, \mathrm{p}$. 206, Lemma 14.4.1], we have

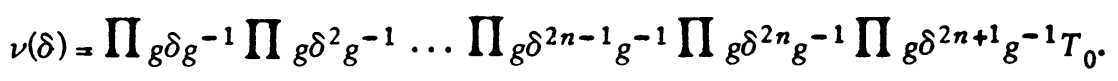

The first product contains an odd number of factors $g \delta g^{-1}$; no $g \delta g^{-1}$ is in $T_{0}$ since $|\delta|=2^{n+2}$ and $T_{0}$ has exponent $2^{n+1}$. Since $\nu(\delta)=1$, some factor in a product other than the first must lie in $T-T_{0} \cdot T-T_{0}=\delta C \cup \delta a_{1} a_{2} C \cup \delta a_{1} C \cup$ $\delta a_{2} C$. The elements of these four cosets of $C$ have orders $2^{n+2}, 2^{n+2}, 2^{2+\xi}$, and $2^{2}+\xi$ respectively. Hence

$$
\begin{array}{ll}
\text { if } \xi=1, \delta^{2^{n-1}} \sim \delta \alpha_{1} y & \text { for some } y \in C ; \\
\text { if } \xi=0, \delta^{2^{n}} \sim \delta \alpha_{1} y & \text { for some } y \in C .
\end{array}
$$

Suppose first that $\xi=1$. If $n \geq 3$, then $\delta^{2 n-1}=\lambda_{1} \mu_{1}$, and $\mathbf{N}_{T}\left(\left(\lambda_{1} \mu_{1}\right\rangle\right)$ is a Sylow 2-subgroup of $\mathbf{N}_{G}\left(\left\langle\lambda_{1} \mu_{1}\right\rangle\right)$. (This is obtained by inspection of the subgroups of order 8 in $T$ whose unique involution is G-conjugate to $\omega$; these are represented up to $T$-conjugacy by $\left\langle\lambda_{1} \mu_{1}\right\rangle,\left\langle\lambda_{1} \mu_{1}^{3}\right\rangle$, and $\left\langle\delta \alpha_{1} y\right\rangle$ where $y \in C_{\text {. }}$ ) Let $\gamma=$ $\delta a_{1} y$; then since $\gamma \sim \lambda_{1} \mu_{1}$, there is $g \in G$ with $\langle\gamma\rangle^{8}=\left\langle\lambda_{1} \mu_{1}\right\rangle$ and $\mathbf{N}_{T}((y))^{8} \leq$ $\mathbf{N}_{T}\left(\left(\lambda_{1} \mu_{2}\right\rangle\right)$. But $\mathbf{N}_{T}\left(\left\langle\lambda_{1} \mu_{1}\right\rangle\right)=\left\langle\mathrm{C}_{T}\left(\lambda_{1} \mu_{1}\right), a_{1} a_{2}\right\rangle$ where $a_{1} a_{2}$ inverts $\lambda_{1} \mu_{1}$; and $\mathbf{N}_{T}(\langle\gamma\rangle)=\left\langle\mathbf{C}_{T}(\gamma), \omega_{1}\right\rangle$ where $\omega_{1}$ raises $\gamma$ to the 5 th power.

If $\xi=1$ and $n=2$, then $|\delta|=16$ and $\delta^{2 n-1}=\delta^{2}=\pi . \mathbf{N}_{T}(\langle\pi\rangle)$ is a Sylow 2-subgroup of $\mathbf{N}_{G}(\langle\pi\rangle)$. Let $\gamma=\delta \alpha_{1} y$; then there is $g \in G$ with $\langle\gamma\rangle^{\boldsymbol{g}}=\langle\pi\rangle$ and $\mathbf{N}_{T}(\langle\gamma\rangle)^{8} \leq \mathbf{N}_{T}((\pi))$. But $\mathbf{N}_{T}((\pi))=\left\langle\mathbf{C}_{T}(\pi), a_{1} a_{2}\right\rangle$ where $a_{1} \alpha_{2}$ cubes $\pi$; and $\mathbf{N}_{T}(\langle y\rangle)=\left\langle\mathbf{C}_{T}(\gamma), \omega_{1}\right\rangle$ where $\omega_{1}$ raises $\gamma$ to the 5 th power.

Now suppose $\xi=0 . \delta^{2^{n}}=\cdot \lambda_{0} \mu_{0}$, and $\mathrm{C}_{T}\left(\lambda_{0} \mu_{0}\right)$ is a Sylow 2-subgroup of 
$\mathbf{C}_{G}\left(\lambda_{0} \mu_{0}\right)$. Let $\gamma=\delta a_{1} y$; since $\gamma \sim \lambda_{0} \mu_{0}$, there is $g \in G$ with $\gamma^{8}=\lambda_{0} \mu_{0}$ and $\mathbf{C}_{T}(\gamma)^{8} \leq \mathbf{C}_{T}\left(\lambda_{0} \mu_{0}\right)$, i.e., $\langle\gamma, \omega\rangle^{8} \leq\langle C, \delta\rangle$. Hence $\left\langle\gamma^{2}, \omega\right\rangle^{8}=\Omega_{1}(\langle C, \delta\rangle)=W$. But $\left(\gamma^{2}\right)^{g}=\omega$, so $\omega^{g}=\omega_{1}$ or $\omega_{2}$, contrary to hypothesis.

We now have some easy fusion results:

(ix) No involution of $T-T_{0}$ is $\sim \omega$.

(x) Let $x \in T-T_{0}$ have $x^{2} \sim \omega$; then $x \sim$ some element of $T_{0}$.

Proof. (x) follows from (ix) by transfer; for if $\nu: G \rightarrow T / T_{0}$ is the transfer homomorphism, then

$$
\nu(x)=\prod g x g^{-1} \prod g x^{2} g^{-1} T_{0},
$$

and all the factors $g x^{2} g^{-1}$ lie in $T_{0}$, by (ix). The first product contains an odd number of $g x g^{-1}$, so since $\nu(x)=1$, some $g x g^{-1}$ lies in $T_{0}$.

To prove (ix), we suppose $z$ is an involution of $T-T_{0}$ with $z \sim \omega$. Then there is $g \in G$ with $z^{8}=\omega$ and $\mathrm{C}_{T}(z)^{8} \leq T$. Now if $\mathrm{C}_{T}(z)$ contains $y$ with $y^{4}$ $=\omega$, then $y^{8} \in T$ gives that $\omega^{8} \in \Phi(\Phi(T)) \leq C$, so $\omega^{g}=\omega$ which contradicts $z^{8}$ $=\omega_{r}$. Such a $y$ exists in $\Delta$ whenever $n \geq 3$. If $n=2$ and $\left[T_{0}: D\right]=4$, then there are elements $a, b$ generating $C$ with $a^{z}=b$ and $b^{z}=a$, and $a b$ or $a b \omega_{1}$ (according as $C$ is Abelian or not) powers to $\omega$ and is centralized by $z$. If $n=$ 2 and $T_{0}=D$, then $T$ has normal $E_{16}$ 's. Suppose $n=2$ and $\left[T_{0}: D\right]=2$. Since $\langle\lambda\rangle$ and $\langle\mu\rangle$ are exchanged by $T / T_{0}$, we can choose $\lambda$ and $\mu$ so that $z$ exchanges $\lambda$ and $\mu$. Then $\pi^{2}=\lambda \mu$ is fixed by $z$, so $1=\left[\pi^{2}, z\right]=[\pi, z]^{\pi}[\pi, z]$ and $\pi$ inverts $[\pi, z]$. As $\Omega_{2}(C)$ is central in $C$, it follows that $[\pi, z] \in W$. But $z^{2}=1$, so $z$ also inverts $[\pi, z]$; therefore $[\pi, z] \epsilon\langle\omega\rangle$, and $\pi$ or $\pi \omega_{1}$ is centralized by $z$.

(xi) Suppose $\left[T_{0}: D\right]=2$. Then $\xi=0$ in (vii), so that $\left[a_{1}, \pi\right]=\lambda$ and $\left[a_{2}, \pi\right]=\mu$.

More generally, the 2-group $T$ given by

$$
\begin{aligned}
T=\langle\langle\alpha, \lambda\rangle \times\langle\beta, \mu\rangle, \pi, \tau: & \alpha^{2}=\lambda^{2^{n}}=\beta^{2}=\mu^{2^{n}}=\tau^{2}=1 ; \alpha \text { inverts } \lambda \\
& \text { and } \beta \text { inverts } \mu ; \alpha^{\tau}=\beta, \lambda^{\tau}=\mu ; \pi^{2}=\lambda \mu, \\
& \left.\pi \text { centralizes } \lambda, \mu \text {, and } \tau ; \alpha^{\pi}=a \lambda \omega_{1}, \beta^{\pi}=\beta \mu \omega_{2}\right\rangle, \\
& \text { where } n \geq 2, \text { and } \omega_{1}=\lambda^{2^{n-1},} \\
& \omega_{2}=\mu^{2^{n-1}}, \omega=\omega_{1} \omega_{2},
\end{aligned}
$$

cannot occur as the Sylow 2-subgroup of a fusion-simple finite group, under the assumptions: $\omega_{1}$ and $\omega_{2} \alpha \omega$; every involution of $\langle\alpha, \lambda\rangle \times\langle\beta, \mu\rangle$ is $G$-conjugate to $\omega$ or $\omega_{1}$; and no involution of $T-\mathbf{C}_{T}\left(\omega_{1}\right)$ is $G$-conjugate to $\omega$.

Proof. We shall first verify that if $\left[T_{0}: D\right]=2$ and $\xi=1$ in (vii), then $T$ is isomorphic to the group described. Let $\tau$ be an involution of $T-T_{0}$, and take 
$\lambda$ and $\mu$ so that $\lambda^{\gamma}=\mu$. Let $\alpha_{1}=\alpha$, and let $\alpha_{1}^{\tau}=\beta$, so that $\beta \in \alpha_{2}\langle\mu\rangle$. There is $\pi \in C-\Delta$ with $\pi^{2}=\lambda \mu$, by (vi); r centralizes $\pi^{2}$, so $\pi$ and $\tau$ both invert $[\pi, r]$, and so $[\pi, r] \epsilon\langle\omega\rangle$; replacing $\pi$ by $\pi \omega_{1}$ if necessary, we may assume $\pi^{2}$ $=\lambda \mu$ and $r$ centralizes $\pi$.

We now consider the given $T$. By transfer, every involution of $T-T_{0}$ is conjugate to an element of $\Omega_{1}\left(T_{0}\right)=\langle\alpha, \lambda\rangle \times\langle\beta, \mu\rangle$, and hence to $\omega$ or $\omega_{1}$. By assumption, then, every involution of $T-T_{0}$ is conjugate to $\omega_{1}$.

We claim that for every involution $x$ of $T-T_{0},\langle x, \omega\rangle$ is $G$-conjugate to $\left\langle\omega_{1}, \omega\right\rangle=W$. Namely, $x \sim \omega_{1}$ implies that there is $g \in G$ with $x^{8}=\omega_{1}$ and $\mathrm{C}_{T}(x)^{8} \leq T_{0}$. As $\omega$ is a square in $\mathrm{C}_{T}(x)$ (e.g., by the action of $x$ on $\langle\lambda, \mu)$ ), $\omega^{8} \in \Phi\left(T_{0}\right)=\langle\lambda, \mu\rangle$, and so $\omega^{8}=\omega$, so that $\langle x, \omega\rangle^{8}=\left\langle\omega_{1}, \omega\right\rangle$.

Now let $H=\left\langle\pi \alpha \beta, \lambda_{0} \mu_{0}\right\rangle \circ\langle W, \tau\rangle$, where $\lambda_{0}=\lambda^{2^{n-2}}$ and $\mu_{0}=\mu^{2^{n-2}}$. Every subgroup $\cong Q_{8} \circ D_{8}$ of $T$ with central involution $G$-conjugate to $\omega$ is $T$-conjugate to $H$. The $H$-conjugacy classes of noncentral involutions of $H$ are represented by $\omega_{1}$ and four elements $x$ of $T-T_{0}$. For any of these $x,\langle x, \omega\rangle \sim w$, so there is $g \in G$ with $\langle x, \omega\rangle^{8}=W$ and $N_{T}(\langle x, \omega\rangle)^{8} \leq T$. Hence $H^{8} \leq T$, and by adjusting $g$ from $T$, we may assume $H^{g}=H$. Therefore $\mathbf{N}_{G}(H)$ acts transitively on the five $H$-conjugacy classes of noncentral involutions of $H$, and so 5 divides $\left|\mathbf{A}_{G}(H)\right|$.

Now $\mathbf{N}_{T}(H)=\left\langle H, \lambda_{0}, \pi^{2^{n-2}}\right\rangle$, and $\pi^{2^{n-2}}$ fixes three of the five classes of noncentral involutions, while $\lambda_{0}$ only fixes one. So $\mathbf{A}_{G}(H) / \operatorname{Inn}(H)$ is a subgroup of $\Sigma_{5}$ whose order is divisible by 5 and whose Sylow 2-subgroups are fourgroups permutation-isomorphic to $\langle(12),(12)(34)\rangle$. But $\Sigma_{5}$ contains no such subgroups.

(xii) $\left[T_{0}: D\right] \leq 2$.

More generally, whenever the Sylow 2-subgroup $T$ of a group $G$ with no nontrivial 2 -factor-group can be described as follows:

$$
\begin{gathered}
D=\langle a, \lambda\rangle \times\langle\beta, \mu\rangle \quad \begin{array}{l}
\text { as above (i.e., each factor is dihedral of order } \\
\\
\left.2^{n+1} \text { where } n \geq 2\right) \text {, with } \lambda^{2^{n-1}}=\omega_{1}, \mu^{2^{n-1}}= \\
\\
\omega_{2}, \text { and } \omega=\omega_{1} \omega_{2}, \text { where } \omega_{1} \chi_{G} \omega ;
\end{array} \\
T=\left\langle D, \rho, \sigma, \tau: \rho^{2}=\lambda, \sigma^{2}=\mu,[\rho, \sigma]=1 \text { or } \omega ;[\alpha, \rho]=\lambda \omega_{1}^{i},[\beta, \sigma]\right. \\
\left.=\mu \omega_{2}^{i} ;[\alpha, \sigma]=\omega_{1}^{j},[\beta, \rho]=\omega_{2}^{j} ; \tau^{2}=1 ; \rho^{\tau}=\sigma, \alpha^{\tau}=\beta\right\rangle, \\
\text { where } i, j \text { are each } 0 \text { or } 1 ;
\end{gathered}
$$

then no involution of $T-T_{0}$ is $\sim \omega$, and hence every $x \in T-T_{0}$ with $x^{2} \sim \omega$ has $x \sim T_{0}$. The elements of order 4 in $T_{0}$ with square $\sim \omega$ (equivalently, square equal to $\omega$, since $\left.\Phi\left(T_{0}\right)=\langle\lambda, \mu\rangle\right)$ are represented by $\lambda_{0} \mu_{0}$ plus the following classes in $T_{0}-\langle\rho, \sigma\rangle$ : 
If $[\rho, \sigma]=\omega$, then

$$
\begin{aligned}
& i=0, j=0: \quad \alpha \beta \rho \sigma \Delta(\text { where } \Delta=\langle\lambda, \mu\rangle) . \\
& i=0, j=1: \quad \text { none. } \\
& i=1, j=0: \quad a \rho \mu_{0}\langle\lambda, W\rangle: \\
& i=1, j=1: \quad \alpha \beta \rho \sigma \Delta, a \rho \mu_{0}\langle\lambda, W\rangle, \text { and } \alpha \beta \rho \Delta .
\end{aligned}
$$

Their centralizers in $T$ are

$$
\begin{aligned}
\mathbf{C}_{T}\left(\alpha \rho \mu_{0}\right) & =\left\langle\alpha \rho \mu_{0}, W, \mu, \beta\right\rangle \quad \text { if } j=1, \\
& =\left\langle\alpha \rho \mu_{0}, W, \mu, \beta \lambda_{0} \sigma\right\rangle \text { if } j=0 \quad \text { (assuming } i=1 \text { ). } \\
\mathbf{C}_{T}(\alpha \beta \rho \sigma) & =\left\langle\alpha \beta \rho \sigma, W, \tau \lambda_{0} \mu_{0}\right\rangle \simeq \mathrm{Z}_{4} \circ D_{8} \quad \text { (assuming } i+j=0 \text { ). } \\
\mathbf{C}_{T}(\alpha \beta \rho) & =\left\langle\alpha \beta \rho, \beta, \mu_{0}\right\rangle \simeq \mathbf{Z}_{4} \times \mathrm{Z}_{2} \times \mathrm{Z}_{2} \quad \text { (assuming } i=j=1 \text { ). }
\end{aligned}
$$

If $[\rho, \sigma]=1$, then

$$
\begin{aligned}
& i=0, j=0: \text { none. } \\
& i=0, j=1: \quad \alpha \beta \rho \sigma \Delta . \\
& i=1, j=0: \quad \alpha \beta \rho \sigma \Delta, \alpha \rho \mu_{0}\langle\lambda\rangle, \text { and } \alpha \rho \mu_{0} \omega\langle\lambda\rangle . \\
& i=1, j=1: \quad a \rho \mu_{0}\langle\lambda\rangle, a \rho \mu_{0} \omega\langle\lambda\rangle, \text { and } \alpha \beta \rho \Delta .
\end{aligned}
$$

Their centralizers in $T$ are

$$
\begin{aligned}
\mathbf{C}_{T}\left(\alpha \rho \mu_{0}\right) & =\mathbf{C}_{T}\left(\alpha \rho \mu_{0} \omega\right)=\left\langle\alpha \rho \mu_{0}, \mu, W, \sigma\right\rangle \text { if } j=0, \\
& =\left\langle\alpha \rho \mu_{0}, \mu, w, \sigma \lambda_{0}, \beta\right\rangle \quad \text { if } j=1 \quad \text { (assuming } i=1 \text { ). } \\
\mathbf{C}_{T}(\alpha \beta \rho \sigma) & =\langle\alpha \beta \rho \sigma, \tau, W\rangle \cong \mathbf{Z}_{4} \circ D_{8} \cdot \\
\mathbf{C}_{T}(\alpha \beta \rho) & \left.=\left\langle\alpha \beta \rho, W, \beta \mu_{0}\right\rangle \cong \mathbf{Z}_{4} \times \mathbf{Z}_{2} \times \mathbf{Z}_{2} \quad \text { (assuming } i=j=1\right) .
\end{aligned}
$$

Proof. We will first establish that if $\left[T_{0}: D\right]=4$, then $T$ can be described as in the assumptions of (xii); and moreover, that notation may be chosen with $\alpha=a_{1}$ and $\beta=a_{2}$ (we shall need this for a transfer argument later in the proof of (xii)).

$\langle\lambda\rangle$ and $\langle\mu\rangle \triangleleft T_{0}$ and are exchanged by $T / T_{0}$. So, if $T$ is an involution of $T-T_{0}, \lambda$ and $\mu \in\langle\lambda\rangle$ and $\langle\mu\rangle$, and $\rho, \sigma \in \mathbf{C}_{T}(V)=C$, can be taken so that

$$
\rho^{\tau}=\sigma ; \rho^{2}=\lambda, \quad \sigma^{2}=\mu ;[\rho, \sigma]=1 \text { or } \omega .
$$

Now $\alpha_{1}^{r} \epsilon a_{2}\langle\mu\rangle$. If $a_{1}^{r} \in a_{2}\left\langle\mu^{2}\right\rangle$, then by replacing $r$ with $r \lambda^{-r} \mu^{r}$ for some $r$ (this does not alter the action of $r$ on $C$ ) we may assume $a_{1}^{r}=a_{2}$. If $a_{1}^{r} \in a_{2} \mu\left\langle\mu^{2}\right\rangle$, 
it will be shown that we can still alter $\tau$ so as to assume $\alpha_{1}^{\tau}=\alpha_{2}$. We need to find the action of $a_{1}$ and $a_{2}$ on $\rho$ and $\sigma$. Now $\left[a_{1}, \rho\right] \in \Delta=\langle\lambda, \mu\rangle \leq \mathbf{Z}(C)$, and so

$$
\left[a_{1}, \rho\right]^{2}=\left[a_{1}, \rho^{2}\right]=\left[\alpha_{1}, \lambda\right]=\lambda^{2} .
$$

Hence $\left[a_{1}, \rho\right]=\lambda w$, for some $w \in W$. But $w \in H_{1}$, so $w \in\left\langle\omega_{1}\right\rangle$. So $\left[\alpha_{1}, \rho\right]=$ $\lambda \omega_{1}^{i}$, where $i=0$ or 1 . Conjugating by $\tau$, we get $\left[\alpha_{1}^{\tau}, \sigma\right]=\mu \omega_{2}^{i}$. But $\alpha_{1}^{\tau} \equiv$ $\alpha_{2} \bmod \langle\mu\rangle$, so has the same action as $\alpha_{2}$ on $\sigma$; so $\left[\alpha_{2}, \sigma\right]=\mu \omega_{2}^{i}$. Also,

$$
\left[a_{1}, \sigma\right]^{2}=\left[a_{1}, \sigma^{2}\right]=\left[a_{1}, \mu\right]=1 \text {, }
$$

so $\left[\alpha_{1}, \sigma\right] \in W ;$ but $\left[\alpha_{1}, \sigma\right] \in H_{1}$, so $\epsilon\left\langle\omega_{1}\right\rangle$, so $\left[\alpha_{1}, \sigma\right]=\omega_{1}^{j} ;\left[\alpha_{2}, \rho\right]=\omega_{2}^{j}$, where $j=0$ or 1 .

Now suppose $a_{1}^{\tau} \in a_{2} \mu\left\langle\mu^{2}\right\rangle$. If $[\rho, \sigma]=1$, then $\tau \rho^{-1} \sigma$ also exchanges $\rho$ and $\sigma$, and is an involution, and sends $\alpha_{1}$ into $a_{2}\left\langle\mu^{2}\right\rangle$, so can be further adjusted as above to get $a_{1}^{\tau}=a_{2}$. If $[\rho, \sigma]=\omega$, then $\tau \rho^{-1} \sigma$ is an involution, but sends $\rho$ to $\sigma \omega$; however, we may replace $\sigma$ by $\sigma \omega$ without altering the defining relations of $T_{0}$. With these adjustments, writing $\alpha=\alpha_{1}$ and $\beta=\alpha_{2}$, we have the $T$ described in the hypotheses.

We now find which elements of $T_{0}-C$ have squares $\sim \omega$. No elements of $D-C$ have this property. Now $T_{0} / \Delta=\langle\alpha \Delta, \beta \Delta, \rho \Delta, \sigma \Delta\rangle$ where $\tau \Delta$ exchanges $\alpha \Delta$ and $\beta \Delta$, and exchanges $\rho \Delta$ and $\sigma \Delta$. So the $T$-classes of cosets of $\Delta$ in $T_{0}$ are represented by $\alpha \Delta, \rho \Delta, \alpha \rho \Delta, \alpha \sigma \Delta ; \alpha \beta \Delta, \alpha \beta \rho \Delta ; \rho \sigma \Delta, \alpha \rho \sigma \Delta ; \alpha \beta \rho \sigma \Delta$. The cosets neither in $D$ nor in $C$ are represented by

$$
\alpha \rho \Delta, \alpha \sigma \Delta ; \alpha \beta \rho \Delta ; \alpha \rho \sigma \Delta ; \alpha \beta \rho \sigma \Delta \text {. }
$$

If $\delta \in \Delta$, then $(\alpha \sigma \delta)^{2} \equiv \lambda \bmod \Delta^{2}$, and $(\alpha \rho \sigma \delta)^{2} \equiv \mu \bmod \Delta^{2}$; so $\alpha \sigma \Delta$ and $\alpha \rho \sigma \Delta$ contain no elements with square equal to $\omega$. Writing $[\rho, \sigma]=\omega^{k}$, we have, for $\delta \in \Delta$,

$$
(\alpha \beta \rho \sigma \delta)^{2}=(\alpha \beta \rho \sigma)^{2}=\omega^{i+j+k},
$$

and $\alpha \beta \rho \sigma \Delta$ is a single $C$-class. Also, $(\alpha \rho \delta)^{2}=\omega_{1}^{i} \delta^{\alpha \rho} \delta$, so $(\alpha \rho \delta)^{2}=\omega$ if and only if $i=1$ and $\delta \in \mu_{0}\langle\lambda, W\rangle$. And $\alpha \rho \mu_{0}\langle\lambda, W\rangle$ is a single $C$-class if $[\rho, \sigma]=\omega$, while it is two $C$-classes, represented by $\alpha \rho \mu_{0}$ and $\alpha \rho \mu_{0} \omega$, if $[\rho, \sigma]=1$.

Note also that the proofs of (ix) and (x) go through under the assumptions of (xii). This completes the proof of the second part of (xii), and we will now use the second part to prove the first part, namely that $\left[T_{0}: D\right] \leq 2$. We shall use transfer to obtain a contradiction from the assumption $\left[T_{0}: D\right]=4$.

By $(\mathbf{x}), \tau \omega_{1} \sim$ some element of $T_{0}$. But $\tau \omega_{1} \not \lambda_{0} \mu_{0}$; for if so, there is $g \epsilon$ $G$ with $\left(\tau \omega_{1}\right)^{8}=\lambda_{0} \mu_{0}$ and $\mathrm{C}_{T}\left(\tau \omega_{1}\right)^{8} \leq \mathrm{C}_{T}\left(\lambda_{0} \mu_{0}\right)=\langle\tau, C\rangle$. In particular, 
$(\alpha \beta)^{8} \in\langle\tau, C\rangle$ but $(\alpha \beta)^{8} \neq \omega$; but by (ix), no involution of $\langle\tau, C\rangle$ but $\omega$ is $\sim \omega$ $\sim \alpha \beta$.

First assume $[\rho, \sigma]=\omega$. By knowing $\mathrm{C}_{T}(z)$ for various $z \in T_{0}$ with $z^{2}=\omega$, we get that if $\tau \omega_{1} \sim \alpha \rho \mu_{0}$, then $\mathbf{C}_{T}\left(\tau \omega_{1}\right)$ and $\mathbf{C}_{T}\left(\alpha \rho \mu_{0}\right)$ are both Sylow 2-subgroups of their centralizers in $G$, so should be isomorphic; but they are not (e.g., they have different exponents). If $\tau \omega_{1} \chi \alpha \rho \mu_{0}$ and $\tau \omega_{1} \chi \lambda_{0} \mu_{0}$, but $\tau \omega_{1} \sim \alpha \beta \rho \sigma$, then $\mathbf{C}_{T}\left(\tau \omega_{1}\right)$ is a Sylow 2-subgroup of $\mathbf{C}_{G}\left(\tau \omega_{1}\right)$, so there is $g \in G$ with

$$
(\alpha \beta \rho \sigma)^{8}=\tau \omega_{1}, \quad \mathrm{C}_{T}(\alpha \beta \rho \sigma)^{8} \leq \mathrm{C}_{T}\left(\tau \omega_{1}\right) .
$$

Now all the $Q_{8}$ 's in $\mathrm{C}_{T}\left(\tau \omega_{1}\right)$ contain $\lambda_{0} \mu_{0}$, so some element of the $Q_{8}$ in $\mathbf{C}_{T}(\alpha \beta \rho \sigma)$ is conjugate to $\lambda_{0} \mu_{0}$. Now all elements of $T-T_{0}$ with square $\omega$ are $T_{0}$-conjugate, so we must have $\alpha \beta \rho \sigma \omega_{1} \sim \lambda_{0} \mu_{0}$; but $\alpha \beta \rho \sigma \omega_{1} \sim \alpha \beta \rho \sigma$ which $\not \lambda_{0} \mu_{0}$ by assumption. Finally, if $\tau \omega_{1} \sim \alpha \rho \mu_{0}$, then $\mathrm{C}_{T}\left(\alpha \rho \mu_{0}\right) \cong \mathbf{Z}_{4} \times \mathbf{Z}_{2} \times \mathbf{Z}_{2}$ is conjugate to a subgroup of $\mathrm{C}_{T}\left(\tau \omega_{1}\right) \cong \mathrm{Z}_{4} \circ D_{2^{n+2}}$, but this is impossible.

Now assume $[\rho, \sigma]=1$. If $\tau \omega_{1} \sim \alpha \rho \mu_{0}$ or $\alpha \rho \mu_{0} \omega$, then $\mathbf{C}_{T}\left(\tau \omega_{1}\right)$ is conjugate to a subgroup of $\mathrm{C}_{T}\left(\alpha \rho \mu_{0}\right)$. But the only involutions which are fourth powers in $\mathbf{C}_{T}\left(\tau \omega_{1}\right)$ and $\mathbf{C}_{T}\left(\alpha \rho \mu_{0}\right)$, respectively, are $\omega$ and $\omega_{2}$ respectively, and $\omega \cdot \mathcal{x} \omega_{2}$. If $\tau \omega_{1} \sim \alpha \beta \rho \sigma$ but not to $\alpha \rho \mu_{0}$ or $\lambda_{0} \mu_{0}$, then there is $g \in G$ with

$$
(\alpha \beta \rho \sigma)^{8}=\tau \omega_{1}, \quad \mathrm{C}_{T}(\alpha \beta \rho \sigma)^{8} \leq \mathrm{C}_{T}\left(\tau \omega_{1}\right),
$$

and we get a contradiction by considering $Q_{8}$ 's, as before. Finally, if $\tau \omega_{1} \sim$ $\alpha \beta \rho$, we get $\mathbf{Z}_{4} \times \mathbf{Z}_{2} \times \mathbf{Z}_{2} \leq \mathbf{Z}_{4} \circ D_{2^{n+2}}$, a contradiction.

(xiii) $\alpha_{1} a_{1}^{\tau} \nsim \omega$.

Proof. By (x), $\tau \omega_{1} \sim$ some element of $T_{0}$. Now by (xi), the only elements of $T_{0}$ whose squares $\sim \omega$ are $T$-conjugate to $\lambda_{0} \mu_{0}$; hence $\tau \omega_{1} \sim \lambda_{0} \mu_{0} \cdot \mathbf{C}_{T}\left(\lambda_{0} \mu_{0}\right)$ is a Sylow 2-subgroup of $\mathbf{C}_{G}\left(\lambda_{0} \mu_{0}\right)$, so there is $g \in G$ with $\left(\tau \omega_{1}\right)^{g}=\lambda_{0} \mu_{0}$ and $\mathbf{C}_{T}\left(\tau \omega_{1}\right)^{g} \leq$ $\mathbf{C}_{T}\left(\lambda_{0} \mu_{0}\right)=\langle C, \tau\rangle$. But $\langle C, \tau\rangle$ contains only one conjugate of $\omega$, namely $\omega$ itself; and $\alpha_{1} a_{1}^{\tau} \in \mathbf{C}_{T}\left(\tau \omega_{1}\right),\left(\alpha_{1} a_{1}^{\tau}\right)^{8} \nsim \omega$.

(xiv) $\alpha_{1} \alpha_{1}^{\tau} \sim \omega_{1}$, and $F=\left\langle a_{1} a_{1}^{\tau}, W\right\rangle$ has $A_{G}(F) \cong \Sigma_{5}$.

Proof. $F$ has precisely five conjugates of $\omega$, by (xiii). These five elements of $F$ are permuted by $A_{G}(F)$, so $A_{G}(F) \leq \Sigma_{5}$.

Now for any $z \in F$ with $z \sim \dot{\omega}$, let $U$ be a Sylow 2-subgroup of $\mathbf{C}_{G}(z)$ which contains $F$; then in $U$ there is an element which conjugates the other four $G$-conjugates of $\omega$ in $F$ in two orbits of size 2. The only way this can hold for all such $z \in F$ is for $A_{G}(F)$ to be transitive on the five conjugates of $\omega$. Hence 5 divides $\left|\mathbf{A}_{G}(F)\right|$. Also, $V / W \leq \mathbf{A}_{G}(F)$, and $\mathbf{A}_{V}(F)$ cannot be normalized by a 3element of $\mathbf{A}_{G}(F)$ (for $\left[F, \lambda_{0}\right]$ has order 2 while $\left[F, \lambda_{0} \mu_{0}\right]$ has order 4). Hence, $A_{G}(F) \cong \Sigma_{5}^{+}$. We get $A_{G}(F) \cong \Sigma_{s}$ by looking at subgroups of the relevant orders in $\Sigma_{5}$; in particular, $\Sigma_{5}$ has no subgroup of order 20 in which the Sylow 2-subgroups are noncyclic. 
Hence, one of the following holds:

(a) $|T|=2^{2 n+3}$ (where $n \geq 3$, else $T$ has normal $E_{16}$ 's), and $T=\left\langle\left\langle a_{1}, \lambda\right\rangle\right.$ $\left.\times\left\langle a_{2}, \mu\right\rangle, \tau\right\rangle$ where $\tau^{2}=1, \lambda^{\tau}=\mu, a_{1}^{\tau}=a_{2} \mu$ (replacing $\tau$ by some $\tau \lambda^{-r} \mu^{\tau}$ if necessary). $\left\langle\alpha_{1}, \alpha_{2}, w\right\rangle$ has automizer $\Sigma_{3} \times \Sigma_{3}$, while $\left\langle\alpha_{1}, \alpha_{2} \mu, w\right\rangle$ has automizer $\Sigma_{5^{\prime}}$

(b) $|T|=2^{2 n+4}$ (where $n \geq 2$ ), and $T=\left\langle\left\langle\alpha_{1}, \lambda\right\rangle \times\left\langle\alpha_{2}, \mu\right\rangle, \pi, \tau\right\rangle$ where $\pi^{2}=$ $\lambda \mu, \pi$ centralizes $\lambda$ and $\mu,\left[\alpha_{1}, \pi\right]=\lambda$, and $\left[\alpha_{2}, \pi\right]=\mu ; \tau^{2}=1, \alpha_{1}^{\tau}=\alpha_{2} \mu, \pi^{\tau}=$ $\pi$, and $\lambda^{\tau}=\mu$ (replacing $r$ by some $\pi \lambda^{-r} \mu^{r}$ if necessary). $\left\langle a_{1}, a_{2}, W\right\rangle$ has automizer $\Sigma_{3} \times \Sigma_{3}$, while $\left\langle a_{1}, a_{2} \mu, w\right\rangle$ has automizer $\Sigma_{s}$.

We will now show that $\operatorname{PSL}_{4}(q)$ (for $q \equiv 3 \bmod 4$ ) and $\operatorname{PSU}_{4}(q)$ (for $q \equiv$ 1 mod 4) have Sylow 2-subgroups such as we have found.

Let $U$ be a 4-dimensional space over GF $(q)$ or GF $\left(q^{2}\right)$ respectively, and write $U=U_{1} \oplus U_{2}$, where in the unitary case, $U_{1}$ and $U_{2}$ are nonsingular orthogonal subspaces of $U$, and $U_{1}$ and $U_{2}$ are 2-dimensional. Let $H_{i}=\mathrm{SL}^{ \pm}\left(V_{i}\right)$ or $U\left(V_{i}\right)$ respectively $(i=1,2)$, where $\mathrm{SL}^{ \pm}$means nonsingular linear transformations with determinant \pm 1 . Let $S_{i}$ be a Sylow 2-subgroup of $H_{i}(i=1,2)$. Then $S_{i}$ are semidihedral, and

$$
S_{i}=\left\langle\delta_{i}, \beta_{i}: \beta_{i}^{2^{r}}=1, \delta_{i}^{2}=\beta_{i}^{2^{r-1}}, \delta_{i}^{-1} \beta_{i} \delta_{i}=\beta_{i}^{-1} \beta_{i}^{2^{r-1}}\right\rangle
$$

where $\operatorname{det}\left(\delta_{i}\right)=1$ and $\operatorname{det}\left(\beta_{i}\right)=-1$.

Let $\sigma$ be an involution exchanging $U_{1}$ and $U_{2}$, and choose $S_{1}$ and $S_{2}$ so that

$$
\delta_{i}^{\sigma}=\delta_{i+1}, \quad \beta_{i}^{\sigma}=\beta_{i+1}
$$

Then $T=\left\langle\delta_{1}, \beta_{1}^{2}, \delta_{2}, \beta_{2}^{2}, \beta_{1} \beta_{2}, \sigma\right\rangle$ is a Sylow 2-subgroup of $G_{1}=\operatorname{SL}_{4}(q)$ or $\mathrm{SU}_{4}(q)$ respectively, and $T /\langle-1\rangle$ is a Sylow 2-subgroup of $G=\operatorname{PSL}_{4}(q)$ or $\mathrm{PSU}_{4}(q)$ respectively.

Let $\delta=\delta_{1} \beta_{1} \delta_{2} \beta_{2} ; \delta$ is an involution of $G_{1}$, and $\delta$ inverts $\Delta=\left\langle\beta_{1}^{2}, \beta_{2}^{2}\right.$, $\left.\beta_{1} \beta_{2}\right\rangle \bmod \langle-1\rangle$. Let $\lambda=\beta_{1} \beta_{2}, \mu=\beta_{1} \beta_{2}^{-1}$; then $\Delta=\langle\lambda, \mu\rangle$, and $\sigma$ centralizes $\lambda$ and inverts $\mu \cdot \lambda^{2^{r-1}}=-1=\mu^{2^{r-1}}$, so $\lambda_{2}=\lambda^{2^{r-2}}$ and $\mu_{2}=\mu^{2^{r-2}}$ are involutions of $G$ which are not conjugate to the involution $\delta_{1}^{2}=\left(\begin{array}{cc}-I & 0 \\ 0 & l\end{array}\right)$ of $G$. ( $-I$ denotes the scalar matrix -1 of degree 2.)

$T_{0}=\langle\Delta, \delta, \sigma\rangle$ is a direct product of two dihedral groups. Let $\gamma=\delta_{1}$. Then $\gamma$ exchanges $\langle\lambda\rangle$ and $\langle\mu\rangle$, and also exchanges $\sigma$ and $\sigma \delta \bmod \Delta$; hence $\left\langle\gamma, T_{0}\right\rangle \simeq$ $D_{2 r}\left\{\mathrm{Z}_{2}\right.$, which is one of the isomorphism types we have obtained.

$G$ has two conjugacy classes of $E_{16}$ 's, one coming from $Q_{8} \circ Q_{8}$ of $G_{1}$ and one coming from $Q_{8} \circ D_{8}$ of $G_{1}$. Namely, let $K=\left\langle\delta_{1}^{2}, \sigma\right\rangle=\left\langle\left(\begin{array}{cc}-1 & 0 \\ 0 & l\end{array}\right),\left(\begin{array}{ll}0 & I \\ I & 0\end{array}\right)\right\rangle \cong D_{8}$; $L=\left\langle\lambda_{2} \mu_{2}, \delta\right\rangle \cong D_{8} ; M=\left\langle\lambda_{2} \mu_{2}, \delta_{1} \delta_{2}\right\rangle \cong Q_{8}$. Then $L$ and $M$ both centralize $K$, and $K \circ L \cong Q_{8} \circ Q_{8}, K \circ M \cong D_{8} \circ Q_{8}$. 
Case 2. For every $a \sim \omega$ in $T_{0}-\mathrm{C}_{T}(V),\left|\alpha W \cap \operatorname{ccl}_{G}(\omega)\right|=2$, and there is $\alpha \sim \omega$ in $T_{0}-C_{T}(V)$ with $\alpha W \cap \operatorname{ccl}_{G}(\omega)=\left\{\alpha, \alpha \omega_{1}\right\}$. As before, we look first at $E_{16}$ 's. Let $\alpha \sim \omega, \alpha \in T_{0}-\mathbf{C}_{T}(V)$, with $\alpha W \cap \operatorname{ccl}_{G}(\omega)=\left\{\alpha, \alpha \omega_{1}\right\}$; let $\beta \sim \omega$, $\beta \in T_{0}-\mathrm{C}_{T}(V)$ with $\beta W \cap \mathrm{ccl}_{G}(\omega)=\left\{\beta, \beta \omega_{2}\right\}$. By Lemmas 5a and 5 applied to $T_{0}$ and $V$, we get that $F=\langle\alpha, \beta, W\rangle \cong E_{16}$.

Suppose $\mathbf{C}_{V}(F)>W$. Then by Lemma 3(iii), $\omega_{1}$ or $\omega_{2}$ is the unique involution of $\Phi\left(\mathbf{C}_{V}(F)\right)$, and is central in $\mathbf{N}_{G}(F)$. If $\omega_{1}$, let $U$ be a Sylow 2-subgroup of $\mathrm{C}_{G}(a)$ (and so of $G$ ) containing $F$, and let $R$ be a normal $Z_{4} \times Z_{4}$ of $U$. Then $C_{F}(R)=\Omega_{1}(R)$ by Lemma $3(i)$, so $\alpha$ and $\omega_{1} \in \Omega_{1}(R)$ and $\Omega_{1}(R)=\left\langle\alpha, \omega_{1}\right\rangle$. But this contains two conjugates of $\omega$, violating $\Omega_{1}(R) \sim W$. For $\omega_{2}$, we argue similarly with $\beta$ instead of $\alpha$.

Hence $\mathrm{C}_{V}(F)=W$, and $V$ induces a four-group on $F$. But $[\alpha, V]$ and $[\beta, V]$ both have order 2, so $\mathrm{C}_{V}(\alpha)$ and $\mathrm{C}_{V}(\beta)$ are distinct groups of order 8; so $V=$ $\langle x, y\rangle$ where $\alpha^{x}=a \omega_{1}$ and $\beta^{x}=\beta, a^{y}=\alpha$ and $\beta^{y}=\beta \omega_{2}$. Then $\langle x, y\rangle$ conjugates $\alpha \beta$ to $\alpha \beta z$ for every $z \in W$; so by the hypothesis of Case $2, \alpha \beta \not \omega$. Therefore, $F$ contains exactly five conjugates of $\omega$.

Now let $\gamma \in F$, with $\gamma \sim \omega$, and let $U$ be a Sylow 2-subgroup of $\mathbf{C}_{G}(\gamma)$ containing $F$. Let $\delta \in F$ with $\delta \sim \omega$ and $\delta \neq \gamma$; then $\delta \notin W(U)=$ the unique normal four-group of $U$. Hence, by Alperin's theorem [1] and the hypothesis of Case 2, $\delta$ is conjugate in $U$ to $\delta w$ for some unique $w \in W(U)^{\#}$. If $\delta \sim \delta \gamma$, then $F$ would contain a four-group all of whose involutions are conjugate to $\omega$, which it does not. Hence $\delta W(U) \cap \operatorname{ccl}_{G}(\omega)=\left\{\delta, \delta \omega_{1}(U)\right\}$ say. There remain two conjugates of $\omega$ in $F$; let $\pi$ be one; since the product of all five of them is 1 , the fifth must be $\pi \omega_{2}(U)$. We have now shown that for any $\gamma \sim \omega$ in $F$, there is $v \in \mathbf{N}_{G}(F)$ which permutes the other four conjugates of $\omega$ in two cycles of length 2. It follows that $A_{G}(F)$ is transitive on the five conjugates of $\omega$. Also, taking $x, y \in V$ as above, $[F, x]$ and $[F, y]$ have order 2 while $[F, x y]$ has order 4 . Hence $A_{G}(F) \npreceq \Sigma_{5}^{+}$, for no 3-element can act fixed-point-freely on $A_{V}(F)$. Hence $A_{G}(F) \cong \Sigma_{5}$. By Lemma 3(iii), $F$ is a Sylow 2-subgroup of $\mathbf{C}_{G}(F)$.

We have shown: For any $F=\langle\alpha, \beta, W\rangle$ where $\alpha, \beta \sim \omega$ and $\alpha W \cap \mathrm{ccl}_{G}(\omega)$ $=\left\{\alpha, \alpha \omega_{1}\right\}$ and $\beta W \cap \mathrm{ccl}_{G}(\omega)=\left\{\beta, \beta \omega_{2}\right\}, A_{G}(F) \cong \Sigma_{5}$ and $F$ is a Sylow 2-subgroup of $\mathrm{C}_{G}(F)$. Also, for any $\gamma \sim \omega$ in $F$, and any Sylow 2-subgroup $U$ of $\mathbf{C}_{G}(\gamma)$ which contains $F$, the remaining four conjugates of $\omega$ in $F$ may be written as $\delta, \delta \omega_{1}(U), \pi$, and $\pi \omega_{2}(U)$, where $\omega_{i}(U) \sim \omega_{i}(i=1,2)$, and $\delta, \pi$ are to $U$ as $\alpha, \beta$ were to $T$. Also, we know what four-group it is which $V(U)$ induces on $F$.

(i) $T-T_{0}$ contains an involution.

Proof. Let $F=\langle a, \beta W\rangle$ as above, and let $U$ be a Sylow 2-subgroup of $G$ which contains a Sylow 2-subgroup $R$ of $\mathrm{N}_{G}(F) .|R|=2^{7}$ and $R / F \cong D_{8}$. $\left[R: R \cap U_{0}\right] \leq 2$, where $U_{0}=\mathbf{C}_{U}(W(U))$. Now $R \cap U_{0}$ centralizes $W(U) \leq F$, and 
the Sylow 2-subgroups of $\Sigma_{\text {S }}$ centralize only a subgroup of order 2 in $F$, so $[R$ : $\left.R \cap U_{0}\right]=2$. It follows that there is $\phi \in R-U_{0}$ such that $\phi^{2} \in \mathrm{C}_{R}(F)=F$, and $\phi$ acts nontrivially on both $W(U)$ and $F / W(U)$; and $\phi F$ contains an involution.

(ii) $\mathbf{C}_{T}(V)>V_{\text {. }}$

Proof. Let $\phi$ be an involution of $T-T_{0}$, let $a \in T_{0}$ with $a W \cap \mathrm{ccl}_{G^{\prime}}(\omega)=$ $\left\{\alpha, \alpha \omega_{1}\right\}$, and let $\beta=a^{\phi}$. Let $F=\langle\alpha, \beta, w\rangle . V$ induces a four-group on $F$, and there are $x, y \in V$ with $\alpha^{x}=\alpha \omega_{1}, \beta^{x}=\beta ; \alpha^{y}=\alpha, \beta^{y}=\beta \omega_{2^{\prime}} T: T_{0}$ interchanges $x W$ and $y W$, hence interchanges $x^{2}$ and $y^{2}$; so $\left\{x^{2}, y^{2}\right\}=\left\{\omega_{1}, \omega_{2}\right\}$. So there are two possibilities for $\mathbf{A}_{F}(V)$ : namely, writing matrices with respect to the basis $\{x, y\}$ of $V$, either

(a) $x^{2}=\omega_{1}$ and $y^{2}=\omega_{2}$, so that $\alpha$ and $\beta$ have the matrices $\left(\begin{array}{ll}3 & 0 \\ 0 & 1\end{array}\right)$ and $\left(\begin{array}{ll}1 & 0 \\ 0 & 3\end{array}\right)$ respectively; or

(b) $x^{2}=\omega_{2}$ and $y^{2}=\omega_{1}$, so that $\alpha$ and $\beta$ have the matrices $\left(\begin{array}{ll}1 & 2 \\ 0 & 1\end{array}\right)$ and $\left(\begin{array}{ll}1 & 0 \\ 2 & 1\end{array}\right)$ respectively.

Now assume $\mathrm{C}_{T}(V)=V$. Then $\langle\phi, F V\rangle$ normalizes $\langle\gamma, W\rangle$, where $\gamma=\alpha \beta$; we will obtain a contradiction by showing $\langle\gamma, W\rangle \triangleleft T$. $\left[T_{0}: F V\right] \leq 2$ by Lemma 4 , and we may assume $\left[T_{0}: F V\right]=2$. Since $\phi$ is an involution, the fixed-point set of $\phi$ on $T_{0} / V$ is a four-group, and in fact induces $\left\langle\left(\begin{array}{ll}3 & 0 \\ 0 & 3\end{array}\right),\left(\begin{array}{ll}1 & 2 \\ 2 & 1\end{array}\right)\right\rangle$.

If (a) holds, then $T_{0}=\langle F V, y\rangle$ where $y$ induces $\left(\begin{array}{cc}1 & 2 \\ 2 & 1\end{array}\right)$ on $V$. If $[a, y]$ and $[\beta, y] \in W$, then $[\gamma, y] \in W$ and $\langle\gamma, W\rangle \triangleleft T$; so we may assume $[\alpha, y] \in V-W$. $\alpha^{2}=1$, so $\alpha$ inverts $[\alpha, y]$ and therefore $[a, y] \in x W$. But $y^{2} \in \mathbf{C}_{V}(y)=W$, so $y$ also inverts $[a, y]$ and therefore $[a, y] \epsilon\langle x y, w\rangle$, which is impossible.

If (b) holds, then $T_{0}=\langle F V, y\rangle$ for some $y$, and we may assume $[a, y] \in V-$ W. Again, $a$ inverts $[a, y]$. But $a$ has the matrix $\left(\begin{array}{ll}1 & 2 \\ 0 & 1\end{array}\right)$ on $V$, and so $a$ inverts no element of $V-W$.

We shall now proceed as in Case 1 to obtain a homocyclic Abelian subgroup of index 1,2 , or 4 in $\mathrm{C}_{T}(V)$. We define, as in Case 1 ,

$$
W(\gamma ; H)=\left\{v \in W: \gamma \sim_{H} \gamma v\right\}
$$

for any involution $\gamma$ of $T_{0}-W$ and any subgroup $H$ of $G$. Also

$$
\begin{aligned}
J\left(\omega_{i}\right) & =\left\{\gamma \in T_{0}-W: \gamma \sim \omega \text { and } W(\gamma ; G)=\left\langle\omega_{i}\right\rangle\right\}, \\
H_{i} & =\left\langle J\left(\omega_{i}\right)\right\rangle, \text { for } i=1,2 .
\end{aligned}
$$

Then $H_{1}$ and $H_{2}$ centralize each other.

(iii) $H_{1}$ contains $\lambda_{0} \in V$ with $\lambda_{0}^{2}=\omega_{1}$.

Proof. Let $a \in J\left(\omega_{1}\right), \beta \in J\left(\omega_{2}\right)$, and let $F=\langle a, \beta, w\rangle$. Then $V=\langle x, y\rangle$ where $\alpha^{x}=\alpha \omega_{1}, \beta^{x}=\beta ; \alpha^{y}=\alpha, \beta^{y}=\beta \omega_{2}$. xy has order 4 , and $x y W$ is the unique $T$-invariant nonidentity element of $V / W$. Since $\mathbf{C}_{T}(V)>V$, it follows that 
$x y$ is a square in $\mathbf{C}_{T}(V)$; say $x y=r^{2}$. Then

$$
a \omega_{1}=\alpha^{x y}=a^{r^{2}}=(\alpha[\alpha, r])^{r}=\alpha[\alpha, r]^{2},
$$

where $[a, r] \in V$. Hence $[a, r]^{2}=\omega_{1}$, and $[a, r]=a a^{r} \in H_{1}$.

(iv) Let $H=\left\langle H_{1}, \omega\right\rangle$, and take $\lambda_{0} \in V \cap H_{1}$ with $\lambda_{0}^{2}=\omega_{1}$. Then there is $A \triangleleft H$ with $\left\langle W, \lambda_{0}\right\rangle \leq A, \mathrm{C}_{H}(A)=A$, and $A \cong \mathrm{Z}_{2^{n}} \times \mathrm{Z}_{2}$ for some $n \geq 2$. The involutions of $J\left(\omega_{1}\right)$ are all congruent $\bmod A$, so that $[H: A]=2$.

Proof. Identical to the proof of (iii) in Case 1.

(v) Let $H, A$ be as in (iv). Then there is $\lambda \in A$ such that $A=\langle\lambda\rangle \times\langle\omega\rangle$; $a$ inverts $\lambda$; and $|\lambda|=2^{n} \geq 4$. Moreover, $a \lambda \in J\left(\omega_{1}\right)$, while $a \omega$ and $a \lambda \omega \notin J\left(\omega_{1}\right)$. Hence $H_{1}=\langle\alpha, \lambda\rangle$; and therefore $\langle\lambda\rangle$ and $\langle\mu\rangle \triangleleft T_{0}$ and are exchanged by $T / T_{0}$, and $T_{0}=F C_{T}(V)$. (Here $\mu$ is to $H^{\phi}$ as $\lambda$ is to $H$, for $\phi \in T-T_{0}$.)

Proof. The existence of $\lambda$ is the same as in (iv), Case 1. If $a \omega$ (or $a \lambda \omega$ ) $\epsilon J\left(\omega_{1}\right)$, then $\left|a W \cap \operatorname{ccl}_{G}(\omega)\right| \geq 3$ (or $\left|a \lambda W \cap \operatorname{ccl}_{G}(\omega)\right| \geq 3$ ), contrary to the hypothesis of Case 2.

(vi) Suppose $\left.T_{0}\right\rangle D=\langle\alpha, \lambda\rangle \times\langle\beta, \mu\rangle$. Then each element of $T_{0} / D$ acts semiregularly on the set of $D$-classes of $E_{16}$ 's of $D$ (and hence $\left[T_{0}: D\right] \leq 4$ ).

Proof. Identical to the proof of (v) (a) of Case 1.

We get the structure of $C$ exactly as in Case 1 , with $\Delta=\langle\lambda, \mu\rangle$ and $C=$ $\mathrm{C}_{T}(V)$ :

(vii) If $T_{0}=D$, then $C=\Delta$.

If $\left[T_{0}: D\right]=2$, then $C$ is Abelian and $\Phi(C)=\left\langle\lambda \mu, \Delta^{2}\right\rangle$.

If $\left[T_{0}: D\right]=4$, then $\Phi(C)=\Delta$, and $C=\langle\rho, \sigma\rangle$ where $\rho^{2^{n}}=\omega_{1}, \sigma^{2^{n}}=\omega_{2}$, and $[\rho, \sigma]=1$ or $\omega$.

The following fusion results are also proved exactly as in Case 1, (ix) and (x):

(viii) No involution of $T-T_{0}$ is $\sim \omega$.

(ix) Let $x \in T-T_{0}$ have $x^{2} \sim \omega$; then $x \sim$ some element of $T_{0}$.

We now determine $T$ completely in case $\left[T_{0}: D\right]=2$.

(x) Suppose $\left[T_{0}: D\right]=2$. Then $|T|=2^{2 n+4}$ (for some $n \geq 2$ ), and $T=$ $\langle\langle a, \lambda\rangle \times\langle\beta, \mu\rangle, \pi, \tau\rangle$ where $\pi^{2}=\lambda \mu, \pi$ centralizes $\lambda$ and $\mu$ and $\tau,[a, \pi]=\lambda$, and $[\beta, \pi]=\mu ; \tau^{2}=1, \alpha^{\tau}=\beta, \lambda^{\tau}=\mu$. Every $E_{16}$ of $T$ has automizer $\Sigma_{5}$ in $G$.

Proof. Let $\alpha \in J\left(\omega_{1}\right)$ and let $\beta=a^{r}$, where $T$ is an involution of $T-T_{0^{\circ}}$ Take $\lambda$ and $\mu$ so that $\lambda^{\tau}=\mu$. Since $C$ is Abelian with $\omega$ as characteristic involution, $C=\langle\lambda, \mu, \pi\rangle$ where $\pi^{2}=\lambda \mu$. $\tau$ then centralizes $\pi^{2}$, so $\pi$ inverts $[r, \pi]$ and so $[\tau, \pi] \in W$. But since $\tau$ is an involution, $\tau$ inverts $[r, \pi]$, so $[\tau, \pi] \in\langle\omega\rangle$. If $[r, \pi]=\omega$, we may replace $\pi$ by $\pi \omega_{1}$ to get

$$
\pi^{2}=\lambda \mu, \quad[\tau, \pi]=1
$$


Then $[\alpha, \pi]^{2}=\left[\alpha, \pi^{2}\right]=[\alpha, \lambda, \mu]=\lambda^{2}$, so $[\alpha, \pi]=\lambda w$ for some $w \in W$. $w \epsilon$ $H_{1}$, so $w \in\left\langle\omega_{1}\right\rangle$, and (via $\tau$ )

$$
[\alpha, \pi]=\lambda \omega_{1}^{\xi}, \quad[\beta, \pi]=\mu \omega_{2}^{\xi} \quad(\xi=0 \text { or } 1) .
$$

Hence $T$ is as in the hypothesis of (xi) of Case 1, so by (xi) of Case $1, \xi=0$. Every $E_{16}$ of $T$ lies in $D=\langle\alpha, \lambda\rangle \times\langle\beta, \mu\rangle$, and is generated by $W, \gamma$, and $\delta$ for some $\gamma \in J\left(\omega_{1}\right)$ and $\delta \in J\left(\omega_{2}\right)$; and we have shown that all such $E_{16}$ 's have automizer $\Sigma_{5}$.

We shall now observe that $\operatorname{PSp}_{4}(q)$ (for $q \equiv 3 \bmod 4$ ) has this $T$ as Sylow 2subgroup, and has the $\Sigma_{5}$ fusion pattern in its $E_{16}$ 's. Let $U$ be a 4-dimensional symplectic space over $\mathrm{GF}(q)$, and let $U=U_{1} \oplus U_{2}$ where $U_{1}$ and $U_{2}$ are 2-dimensional nonsingular orthogonal symplectic spaces. Let $H_{i}=\operatorname{SL}\left(V_{i}\right)=\operatorname{Sp}\left(V_{i}\right)$ $(i=1,2)$. Sylow 2-subgroups of $H_{i}$ are $\left\langle\delta_{i}, \beta_{i}^{2}\right\rangle$, a quaternion group, in the notation for $\mathrm{SL}^{\ddagger}\left(V_{i}\right)$ which we used following Case 1.

Let $\sigma$ be an involution exchanging $U_{1}$ and $U_{2}$, and assume $\delta_{i}^{\sigma}=\delta_{i+1}$, $\left(\beta_{i}^{2}\right)^{\sigma}=\beta_{i+1}^{2}$. Then $T=\left\langle\delta_{1}, \beta_{1}^{2}, \delta_{2}, \beta_{2}^{2}, \sigma\right\rangle$ is a Sylow 2-subgroup of $G_{1}=\operatorname{SP}_{4}(q)$, and $T /\langle-1\rangle$ is a Sylow 2-subgroup of $G=\operatorname{PSp}_{4}(q)$.

Let $\lambda=\beta_{1}^{2} \beta_{2}^{2}, \mu=\beta_{1}^{2} \beta_{2}^{-2}$. Then $\sigma$ centralizes $\lambda$ and inverts $\mu$, and $\delta=$ $\delta_{1} \delta_{2}$ inverts $\lambda$ and $\mu$. Let $\pi=\beta_{1}^{2}$. Then $\pi^{2}=\lambda \mu$, and

$$
\begin{aligned}
& \pi^{\sigma}=\left(\beta_{1}^{2}\right)^{\sigma}=\beta_{2}^{2}=\beta_{1}^{2}\left(\beta_{1}^{-2} \beta_{2}^{2}\right)=\pi \mu^{-1}, \\
& \pi^{\sigma \delta}=\beta_{2}^{-2}=\beta_{1}^{2}\left(\beta_{1}^{-2} \beta_{2}^{-2}\right)=\pi \lambda^{-1}, \quad \pi \text { centralizes } \lambda \text { and } \mu \cdot
\end{aligned}
$$

Hence $\langle\sigma \delta, \lambda, \sigma, \mu, \pi\rangle=T_{0}$. Let $\gamma=\delta_{2}$. Then $\gamma$ centralizes $\pi$ and exchanges $\lambda$ and $\mu$, and exchanges $\sigma$ and $\sigma \delta$. So this $T$ has the correct isomorphism type.

It is well known that $\operatorname{PSp}_{4}(q) \cong O_{5}(q)$. Now any $E_{16}$, say $F$, of a 5-dimensional linear group of matrices with determinant 1 , has the $\Sigma_{s}$ fusion pattern (where the field has odd characteristic); for $F$ can be simultaneously diagonalized, so that each $f \in F$ has the form $\operatorname{diag}(a, b, c, d, e)$ where $a, b, c, d, e$ are each \pm 1 (and not all are 1). Since $\operatorname{det}(f)=1$, two or four entries must be -1 , and there are at most $\left(\begin{array}{l}5 \\ 2\end{array}\right)=10$ with two -1 's, and $\left(\begin{array}{l}5 \\ 1\end{array}\right)=5$ with four -1 's; there are 15 altogether, so we have 10 of the first kind and 5 of the second kind. $F$ admits $\Sigma_{s}$ acting on the five simultaneous eigenvectors, so the geometry of the 10 and 5 must be correct.

We now look at the case $\left[T_{0}: D\right]=4$. First we will exhibit examples where such a $T$ occurs. Namely, $\mathrm{SL}_{s}(q)($ for $q \equiv 3 \bmod 4)$ and $\mathrm{SU}_{5}(q)($ for $q \equiv 1 \bmod 4)$ have $D_{2^{n+2}}^{+} \backslash \mathrm{Z}_{2}$ as their Sylow 2-subgroups, where $2^{n}$ is the highest power of 2 to divide $q+1$ or $q-1$ respectively. (For these are the Sylow 2-subgroups of $\mathrm{GL}_{4}(q)$ and $\mathrm{GU}_{4}(q)$, as in Fong and Carter [5]; and the Sylow 2-subgroup of 
the general group in dimension 4 becomes that of the special group in dimension 5 by adjustment of the fifth coordinate so that the determinant is 1 , in each case.) $\operatorname{PSL}_{5}(q)$ and $\operatorname{PSU}_{5}(q)$ have the same Sylow 2-subgroups as the linear groups. The above remark gives that all $E_{16}$ 's must have the $\Sigma_{5}$ fusion pattern in these groups.

We shall show that if $\left[T_{0}: D\right]=4$, then $T$ must be isomorphic to the Sylow 2-subgroups of $\operatorname{PSL}_{5}(q)$ and $\operatorname{PSU}_{5}(q)$ as described above.

The first step is to show that $T$ can be described as in (xii) of Case 1 , so that we can use the calculation from (xii) of Case 1. Take an involution $\tau \in T-$ $T_{0}$, let $a \in J\left(\omega_{1}\right)$, and let $\beta=\alpha^{\tau}$. We may assume $\lambda^{\tau}=\mu$. Now by (vii), $\mathbf{C}_{T}(V)$ $=\langle\rho, \sigma\rangle$ where $\rho^{2}=\lambda, \sigma^{2}=\mu$, and $[\rho, \sigma]=\omega^{k}$ for $k=0$ or 1 . Then $\rho^{\tau}=\sigma x$ for some $x \in W$. We may replace $\sigma$ by $\sigma x$ (and $\alpha, \beta, \rho$ by $\alpha, \beta, \rho$ ) without altering the relations they satisfy; so we shall assume $\rho^{\tau}=\sigma$. Then we get $[\alpha, \rho]=\lambda \omega_{1}^{i}$ and $[a, \sigma]=\omega_{1}^{j}$ as in the proof of (xii) of Case 1 ; applying $\tau$, we get $[\beta, \sigma]=$ $\mu \omega_{2}^{i}$ and $[\beta, \rho]=\omega_{2}^{j}$.

For reference, we repeat the description of $T$ :

$$
\begin{aligned}
T=\left\langle\alpha, \rho, \beta, \sigma, \tau: \alpha^{2}=\beta^{2}=\rho^{2^{n+1}}=\sigma^{2^{n+1}}=\tau^{2}=1 ;\right. \\
\rho^{2^{n}}=\omega_{1}, \sigma^{2^{n}}=\omega_{2} ;[\rho, \sigma]=\omega^{k} ;[\alpha, \rho]=\rho^{2} \omega_{1}^{i},[\beta, \sigma]=\sigma^{2} \omega_{2}^{i} ; \\
\left.\quad[\alpha, \sigma]=\omega_{1}^{j},[\beta, \rho]=\omega_{2}^{j} ; \rho^{\tau}=\sigma, \alpha^{\tau}=\beta\right\rangle .
\end{aligned}
$$

Here $i, j, k$ are each 0 or 1 . We have $\lambda=\rho^{2}, \mu=\sigma^{2}$, and $D=\langle\alpha, \lambda\rangle \times\langle\beta, \mu\rangle$.

Note that $\langle\alpha, \rho\rangle$ and $\langle\beta, \sigma\rangle$ are dihedral if $i=0$ and semidihedral if $i=1$. The Sylow 2-subgroups of $\operatorname{PSL}_{g}(q)$ and $\operatorname{PSU}_{5}(q)$ are the $T$ with $k=0, i=1$, and $j=0$; we need to show that these values of $i, j, k$ must hold, and this will be done by transfer arguments.

(xi) $j=0$ (i.e., $\alpha$ centralizes $\sigma$ and $\beta$ centralizes $\rho$ ).

Proof. Suppose $j=1$, so that $\beta^{\rho}=\beta \omega_{2}$. Then $\mathrm{ccl}_{T_{0}}(\beta)=\beta\langle\mu\rangle$, and $\mathbf{C}_{T}(\beta)=$ $\mathbf{C}_{T}(\beta \omega)=\langle\beta\rangle \times\left\langle\alpha, \rho \mu_{0}, W\right\rangle$, where $\left(\rho \mu_{0}\right)^{2^{n}}=\omega_{1}$ and $\left(\rho \mu_{0}\right)^{a}=\left(\rho \mu_{0}\right)^{-1} \omega_{1}^{i} \omega_{2}$, so that $\Phi\left(\mathbb{C}_{T}(\beta)\right)=\left\langle\rho^{2}, W\right\rangle$. Now $\beta \omega \sim \omega_{2}$, so there is $g \in G$ with $(\beta \omega)^{8}=\omega_{2}$ and $\mathrm{C}_{T}(\beta \omega)^{8} \leq T_{0}$. Hence $W^{\mathcal{B}} \leq \Phi\left(\mathrm{C}_{T}(\beta \omega)\right)^{g} \leq \Phi\left(T_{0}\right)=\langle\lambda, \mu\rangle$, so $W^{\mathcal{B}}=W$; but this contradicts $(\beta \omega)^{8}=\omega_{2^{\prime}}$

(xii) $i=1$ (i.e., $\langle\alpha, \rho\rangle$ and $\langle\beta, \sigma\rangle$ are semidihedral).

Proof. Suppose $i=0$. Let $a^{*} \in \alpha \rho\left\langle\rho^{2}\right\rangle$; then no $x \in \alpha^{*} W$ is $\sim \omega$, for if so, then $x \in J\left(\omega_{1}\right)$ since, by the hypothesis of Case $2,|W(x ; G)|=2$, but $W(x ; T)=$ $\left\langle\omega_{1}\right\rangle$, so $W(x ; G)=\left\langle\omega_{1}\right\rangle$. As we have $\left\langle J\left(\omega_{1}\right)\right\rangle=\left\langle a, \rho^{2}\right\rangle, x \notin J\left(\omega_{1}\right)$. Also, no involution $\gamma$ of $\alpha \beta\langle\rho, \sigma\rangle$ can be $\sim \omega$, since $W(\gamma ; G)=W$.

Hence, the $E_{16}$ 's $\langle a, \beta, w\rangle,\left\langle\alpha^{*}, \beta, w\right\rangle$, and $\left\langle\alpha^{*}, \beta^{*}, w\right\rangle$ (where $\beta^{*} \in \beta \sigma\left\langle\sigma^{2}\right\}$ ) 
contain respectively 5,3 , and 1 conjugates of $\omega$. They also represent the $T$-conjugacy classes of $E_{16}$ 's of $T$; so any two $E_{16}$ 's of $T$ which are $G$-conjugate are $T$-conjugate.

We claim $\alpha \rho \sim \omega_{1}$. Namely, let $M=\langle D, \tau, \rho \sigma\rangle$, maximal in $T$, so $\alpha \sim M$ by transfer. (We shall write " $x \sim H$ " to mean " $x$ is conjugate to some element of $H^{\prime \prime}$.) The only involutions of $M-D$ are conjugate to $\tau$ or (if $[\rho, \sigma]=1$ ) to $\alpha \beta \rho a$. All involutions of $D$ are $\sim W$. So if $a \rho \not \omega_{1}$, then either $a \rho \sim \tau \not W$ or $a \rho \sim$ $\alpha \beta \rho \sigma \not \alpha \tau$ and $W$.

$$
\begin{aligned}
& \mathbf{C}_{T}(\alpha \rho)=\langle a \rho, W, \beta, \sigma\rangle, \text { of order } 2^{n+4}, \text { if }[\rho, \sigma]=1 ; \\
&=\left\langle\alpha \rho, W, \beta, \sigma^{2}\right\rangle, \text { of order } 2^{n+3}, \text { if }[\rho, \sigma]=\omega . \\
& \mathrm{C}_{T}(\tau)=\langle\tau\rangle \times\left\langle\alpha \beta, \rho \sigma \omega_{1}^{k}\right\rangle, \text { of order } 2^{n+3} . \\
&\left|\mathrm{C}_{T}(\alpha \beta \rho \sigma)\right|=2^{5} \text { and } \mathrm{C}_{T}(\alpha \beta \rho \sigma) \geq\langle W, \tau\rangle \text { or }\left\langle W, \tau \lambda_{0} \mu_{0}\right\rangle \text { according as }[\rho, \sigma]=
\end{aligned}
$$
1 or $\omega$.

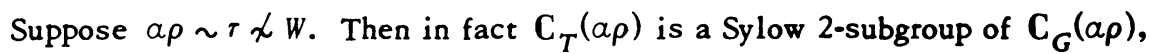
so $\mathrm{C}_{T}(\tau)$ is conjugate to a subgroup of $\mathrm{C}_{T}(\alpha \rho)$. But the only involution which is a square in $\mathbf{C}_{T}(\alpha \rho)$ is $\omega_{2}$, and $\omega$ is a square in $\mathbf{C}_{T}(\tau)$, and $\omega \chi \omega_{2}$. So we may assume $a \rho \sim \alpha \beta \rho \sigma$. Then $\mathbf{C}_{T}(\alpha \beta \rho \sigma)$ is conjugate to a subgroup of $\mathbf{C}_{T}(\alpha \rho)$; but $\omega$ is also a square in $\mathbf{C}_{T}(\alpha \beta \rho \sigma)$.

Hence $\alpha \rho \sim \omega_{1}$, and there is $g \in G$ with $(\alpha \rho)^{g}=\omega_{1}$ and $\mathbf{C}_{T}(\alpha \rho)^{g} \leq T_{0}$. We may adjust $g$ from $T$ so that $(\alpha \rho)^{g} \in W$ and $K^{g}=K$, where $K=\langle a \rho, \beta, W\rangle$. But $g$ normalizes $\left\langle\operatorname{ccl}_{G}(\omega) \cap K\right\rangle=\left\langle\beta, \beta \omega_{2}, \omega\right\rangle=\langle\beta, W\rangle$, contrary to $\alpha \rho \notin\langle\beta, W\rangle$ and $(\alpha \rho)^{g} \in\langle\beta, w\rangle$.

(xiii) $k=0$.

Proof. Suppose $k=1$. We then note that $\left(\alpha \rho \mu_{0}\right)^{2}=\omega_{r}$. We argue that $\alpha \rho \mu_{0}$ $\sim M=\langle D, \tau, \rho \sigma\rangle$. Namely, the only involutions of $T-M$ lie in $T-T_{0}$ (and so are not conjugate to $\omega$ by (viii)), since $\rho\langle D, \rho \sigma\rangle$ contains no involutions. Hence, if $\nu$ is the transfer homomorphism from $G$ to $T / M$, then

$$
\nu\left(\alpha \rho \mu_{0}\right)=\prod g\left(\alpha \rho \mu_{0}\right) g^{-1} \prod g \omega g^{-1} M=\prod g\left(\alpha \rho \mu_{0}\right) g^{-1} M .
$$

Since the last product contains an odd number of factors, $\alpha \rho \mu_{0} \sim M$.

By (xii) of Case 1 , the only elements of $M$ whose squares are $G$-conjugate to $\omega$ are $T$-conjugate to $\lambda_{0} \mu_{0}$ or $\tau \omega_{1}$.

We argue that $\alpha \rho \mu_{0} \not \lambda_{0} \mu_{0}$. Namely, if so, then there is $g \in G$ with $\left(\alpha \rho \mu_{0}\right)^{g}$ $=\lambda_{0} \mu_{0}$ and $\mathrm{C}_{T}\left(\alpha \rho \mu_{0}\right)^{g} \leq \mathbf{C}_{T}\left(\lambda_{0} \mu_{0}\right)$. Now

$$
\begin{aligned}
& \mathrm{C}_{T}\left(\alpha \rho \mu_{0}\right)=\left\langle\alpha \rho \mu_{0}\right\rangle \times\left\langle\alpha \rho \mu_{0} \beta \sigma \lambda_{0}, \mu\right\rangle \cong \mathrm{Z}_{4} \times D_{2^{n+1}} ; \\
& \mathrm{C}_{T}\left(\lambda_{0} \mu_{0}\right)=\langle\tau, \rho, \sigma\rangle,
\end{aligned}
$$


so $\mathbf{C}_{T}\left(\lambda_{0} \mu_{0}\right)$ contains only one $Z_{4} \times Z_{4}$, namely $V$. So $\left(\alpha \rho \mu_{0}\right)^{8}=\lambda_{0} \mu_{0}$ and $\mu_{0}^{8}$ $\epsilon V$. Hence $\mu_{0}^{g} \in \lambda_{0} W$ or $\mu_{0} W$, and we have $\left\langle a \rho, \mu_{0}\right\rangle \sim V$ and $\alpha \rho \sim \lambda_{0}$ or $\mu_{0}$.

Now $(\alpha \rho)^{\sigma}=\alpha \rho \omega,(\alpha \rho)^{\lambda_{0}}=\alpha \rho \omega_{1}$, so $\alpha \rho$ is conjugate to every element of $\alpha \rho W$. Therefore in $V,(\alpha \rho)^{8}$ is conjugate to every element of $(\alpha \rho)^{8} W$. We shall show that neither $\lambda_{0}$ nor $\lambda_{0} \omega$ is conjugate to its product with $\omega_{2}$, which will be the desired contradiction. Suppose $\lambda_{0} \sim \lambda_{0} \omega_{2}$; then there is $g \in G$ with $\lambda_{0}^{g}=$ $\lambda_{0} \omega_{2}$ and $\mathbf{C}_{T}\left(\lambda_{0}\right)^{8}=\mathbf{C}_{T}\left(\lambda_{0} \omega_{2}\right)$, i.e., $\langle\beta, \rho, \sigma\rangle^{8}=\langle\beta, \rho, \sigma\rangle$. Hence $\langle\rho, \sigma\rangle^{8}=\langle\rho, \sigma\rangle$, for $\langle\rho, \sigma\rangle$ is the only subgroup of index 2 in $\langle\beta, \rho, \sigma\rangle$ whose derived group contains an involution $G$-conjugate to $\omega$. Now since $\omega \chi \omega_{1}, A_{G}(\langle\rho, \sigma\rangle)$ is a 2 group and so its action on $V$ is just that of $T$. But $\lambda_{0}$ is not $T$-conjugate to $\lambda_{0} \omega_{2}$.

Therefore $\alpha \rho \mu_{0} \not \lambda_{0} \mu_{0}$, so $\alpha \rho \mu_{0} \sim \tau \omega_{1} \not \lambda_{0} \mu_{0}$. Then $\mathbf{C}_{T}\left(\tau \omega_{1}\right) \cong \mathbf{Z}_{4} \circ$ $D_{2^{n+2}}$ and $\mathrm{C}_{T}\left(\alpha \rho \mu_{0}\right) \cong \mathrm{Z}_{4} \times D_{2^{n+1}}$ should be $G$-conjugate, which is impossible.

Case 3. There is $a \sim \omega$ in $T_{0}-C_{T}(V)$, and for every sucb $a, a W \cap \operatorname{ccl}_{G}(\omega)$ $=\{a, \alpha \omega\}$. The basic strategy here is most simply explained by doing first the case where $V=\mathbf{C}_{T}(V)$.

Let $V=\langle a, b\rangle$, where $a^{2}=\omega \in \Omega_{1}(\mathrm{Z}(T))$. Then the matrix-group, with respect to the basis $\{a, b\}$ of $V$, induced on $V$ by $T$ is contained in $\left\langle B^{+},\left(\begin{array}{ll}1 & 0 \\ 1 & 1\end{array}\right)\right\rangle$, where $B^{+}$denotes the subgroup of Aut $(V)$ which fixes $W$ elementwise. Note that $\mathrm{C}_{\mathrm{O}^{+}}\left(\left(\begin{array}{ll}1 & 0 \\ 1 & 1\end{array}\right)\right)=\left[B^{+},\left(\begin{array}{ll}1 & 0 \\ 1 & 1\end{array}\right)\right]=\left\langle\left(\begin{array}{ll}1 & 0 \\ 2 & 1\end{array}\right),\left(\begin{array}{ll}3 & 0 \\ 0 & 3\end{array}\right)\right\rangle$.

(i) No involution can induce $\left(\begin{array}{ll}1 & 0 \\ 2 & 1\end{array}\right)$ on $V$.

Proof. Let $\gamma$ be an involution of $T_{0}$ inducing $\left(\begin{array}{ll}1 & 0 \\ 2 & 1\end{array}\right)$ on $V$; we shall show $\langle\gamma, W\rangle \triangleleft T$. Since $\left(\begin{array}{ll}1 & 0 \\ 2 & 1\end{array}\right)$ is central in $A_{T}(V)$, we have $[y, t] \in V=\mathbf{C}_{T}(V)$ for each $t \in T$. But $\gamma^{2}=1$, so $\gamma$ inverts $[\gamma, t]$, and the only elements of $V$ which $\gamma$ inverts are those of $W$. So $[\gamma, t] \in W$ for any $t \in T$, and $\langle\gamma, W\rangle \triangleleft T$.

(ii) $T_{0}$ contains $\alpha \sim \omega$ inducing $\left(\begin{array}{ll}3 & 0 \\ 0 & 1\end{array}\right)$ on $V$. For any $\phi \in T-T_{0}, a^{\phi}$ induces $\left(\begin{array}{ll}3 & 0 \\ 2 & 1\end{array}\right)$, and $\left(a a^{\phi}\right)^{2} \in a W$.

Proof. The only automorphisms possible for $\alpha$ with $\operatorname{ccl}_{G}(\alpha) \cap \alpha W=\{\alpha, \alpha \omega\}$ are $\left(\begin{array}{ll}1 & 0 \\ 2 & 1\end{array}\right),\left(\begin{array}{ll}3 & 0 \\ 0 & 1\end{array}\right)$, and $\left(\begin{array}{ll}3 & 0 \\ 2 & 1\end{array}\right)$, and $T / T_{0}$ exchanges $\left(\begin{array}{ll}3 & 0 \\ 0 & 1\end{array}\right)$ and $\left(\begin{array}{ll}3 & 0 \\ 2 & 1\end{array}\right)$ in Aut $(V)$. $\left(\begin{array}{ll}1 & 0 \\ 2 & 1\end{array}\right)$ is impossible by (i), so $a$ exists as claimed.

$a \alpha^{\phi}$ induces $\left(\begin{array}{cc}1 & 0 \\ 2 & 1\end{array}\right)$ on $V$, and $\left(a \alpha^{\phi}\right)^{2} \in \mathbf{C}_{V}\left(\alpha \alpha^{\phi}\right)=\langle a, W\rangle$. If $\left(\alpha \alpha^{\phi}\right)^{2} \in W$, then $a a^{\phi} V$ would contain an involution, contrary to (i).

(iii) No element of $T_{0}$ inverts $V$. Hence $A_{T_{0}}(V)=\left\langle\left(\begin{array}{ll}3 & 0 \\ 0 & 1\end{array}\right),\left(\begin{array}{ll}1 & 0 \\ 2 & 1\end{array}\right)\right\rangle$ (for if larger, it contains $\left(\begin{array}{ll}3 & 0 \\ 0 & 3\end{array}\right)$ since it is $\left(\begin{array}{ll}1 & 0 \\ 1 & 1\end{array}\right)$-invariant).

Proof. This follows from $\left(\alpha \alpha^{\phi}\right)^{2} \in a W$.

Namely, suppose $\delta \in T_{0}$ inverts $V$. Then $\delta^{a}=\delta s$ for some $s \in V$, and

$$
\delta=\delta^{a^{2}}=(\delta s)^{a}=\delta s s^{\alpha} ;
$$

hence $\alpha$ inverts $s$, so $s \in\langle a, W\rangle$. $\langle a, W\rangle \triangleleft T$, so for all $t \in T, s s^{t} \in W$. 
Let $\gamma=\left(a \alpha^{\phi}\right)^{2}$. We will compute $\delta^{\gamma}$ in two different ways. We shall need to know $\delta^{a \phi}$. Now $\delta=\delta^{\phi} x$ for some $x \in V$, and

$$
\begin{aligned}
\delta^{a^{\phi}} & =\left(\delta^{\phi} x\right)^{\alpha^{\phi}}=\left(\delta^{\phi}\right)^{a^{\phi}} x^{a^{\phi}}=\left(\delta^{\alpha}\right)^{\phi} x^{a^{\phi}} \\
& =(\delta s)^{\phi} x\left[x, \alpha^{\phi}\right]=\delta\left[x, a^{\phi}\right]_{s}^{\phi} .
\end{aligned}
$$

Hence

$$
\delta^{\alpha a^{\phi}}=(\delta s)^{a^{\phi}}=\left(\delta\left[x, \alpha^{\phi}\right]_{s}^{\phi}\right) s^{\alpha^{\phi}}=\delta\left[x, \alpha^{\phi}\right]_{s}{ }^{\phi}\left[s, \alpha^{\phi}\right] .
$$

Now $\left[x, a^{\phi}\right],\left[s, a^{\phi}\right]$, and $s^{\phi} s$ all lie in $W$; hence

$$
\delta^{a a^{\phi}}=\delta w \text {, for some } w \in W \text {. }
$$

Also, $\gamma=a u$ for some $u \in W$. Hence $\delta^{\gamma}=(\delta w)^{a a \phi}=(\delta w) w=\delta$; but $\delta^{\gamma}=\delta^{a u}=$ $\delta a^{2}$. This contradiction establishes (iii).

(iv) Notation can be taken in T. so that

$$
\begin{aligned}
T=\langle a, b, y, \alpha, \phi: & \langle a, b\rangle \cong \mathrm{Z}_{4} \times \mathbf{Z}_{4} ; y^{2}=a, b^{y}=a^{2} b ; \\
& a^{2}=1, y^{a}=y^{-1}, b^{\alpha}=b ; \\
& \left.\phi^{2}=1, y^{\phi}=y^{-1}, b^{\phi}=a^{-1} b, a^{\phi}=a y\right\rangle .
\end{aligned}
$$

Proof. $A_{T}(V)=\left\langle\cong,\left(\begin{array}{ll}3 & 0 \\ 0 & 1\end{array}\right),\left(\begin{array}{ll}1 & 0 \\ 2 & 1\end{array}\right)\right\rangle$ for some $\supseteq$ which does not centralize $W$. $\left.\Omega^{2} \in \mathbf{C}_{\vartheta_{+}}\left(\begin{array}{ll}1 & 0 \\ 1 & 1\end{array}\right)\right) \cap \mathbf{A}_{T_{0}}(V)=\left\langle\left(\begin{array}{ll}1 & 0 \\ 2 & 1\end{array}\right)\right\rangle$, and if $\Omega^{2}=\left(\begin{array}{ll}1 & 0 \\ 2 & 1\end{array}\right)$ then $\left(\Omega\left(\begin{array}{ll}3 & 0 \\ 0 & 1\end{array}\right)\right)^{2}=1$. So there is $\supseteqq \in \mathbf{A}_{T}(V)-\mathbf{A}_{T_{0}}(V)$ with $\bigcap^{2}=1$. Now $\left(\begin{array}{ll}1 & 0 \\ 1 & 1\end{array}\right) B^{+}$contains four involutions, all conjugate in $B^{+}$. So by making changes of basis in $V$ corresponding to a similarity-matrix $\in \mathfrak{B}^{+}$, we may assume that $\cong=\left(\begin{array}{ll}3 & 0 \\ 3 & 1\end{array}\right)$. $\mathfrak{B}^{+}$is Abelian, so this change of basis will not affect the matrices induced by $T_{0}$ on $V$.

$\bigcap^{2} \in \mathbf{C}_{T}(V)=V$, and also $\Omega^{2} \in \mathbf{C}_{V}(\Omega)=\left\langle a b^{2}\right\rangle$; the coboundary group of $\supseteq$ on $V$ is $\left\langle a b^{2}\right\rangle$, so there is an involution $\phi$ inducing $\supseteqq$.

$a^{2}=\phi^{2}=1$, and $a \alpha^{\phi}=[a, \phi]=(\alpha \phi)^{2}=y$ say induces $\left(\begin{array}{ll}1 & 0 \\ 2 & 1\end{array}\right)$ on $V$. Both $\phi$ and $\alpha$ invert $y$, so $y^{2} \in\left\{a, a^{-1}\right\}$, by (ii). If $y^{2}=a^{-1}$, replace $a$ by $a^{-1}$ and $b$ by $b^{-1}$, which leaves all matrices unchanged.

So, writing $a \phi=\pi$, we can write $T$ as

$$
T=\left\langle a, \pi, b: a \text { inverts } \pi \text { and centralizes } b ; a^{2}=\pi^{16}=b^{4}=1 ; \pi^{b}=\pi^{5}\right\rangle \text {. }
$$

In particular, $T^{\prime}$ is cyclic. If follows from Chabot [6] that $T$ cannot be the Sylow 2-subgroup of a fusion-simple group. However, a direct argument is easy via Grün's first theorem. Namely, $G$ has no nontrivial 2-factor-group and $\mathbf{N}_{G}(T)=T C_{G}(T)$, so by Grün's first theorem (M. Hall [10, p. 214]), $T$ is generated 
by $G$-conjugates of elements of $T^{\prime} . T^{\prime}=\left\langle\pi^{2}\right\rangle$. We will show that five nonidentity cosets of $\Phi(T)=\left\langle\pi^{2}, b^{2}\right\rangle$ in $T$ must fail to contain conjugates of elements of $\left\langle\pi^{2}\right\rangle$, which implies that $T$ is not generated by conjugates of elements of $T^{\prime}$.

$\{1, b, \pi, \pi b, a, a b, a \pi, a \pi b\}$ is a transversal to $\Phi(T)$ in $T$.

The elements of $\pi \Phi(T) \cup \pi b \Phi(T)$ have order 16 , so cannot be conjugate to elements of $\left\langle\pi^{2}\right\rangle$.

If $x \in a b \Phi(T)$, then $x^{2}=b^{2}$ or $b^{2} \pi^{8}=\omega_{1}$ or $\omega_{2^{\prime}}$. So if $x \sim z \in\left\langle\pi^{2}\right\rangle$, then $x^{2} \sim z^{2}$ gives $\omega_{1} \sim \omega$.

If $x \in a \pi b \Phi(T)$, then $x^{2}=a b^{2}$ or $a^{-1} b^{2}$ (note $a=\pi^{4}$ ). So if $x \sim z \in\left\langle\pi^{2}\right\rangle$, then $a b^{2} \sim a$. But $a b^{2} \chi a$, for

$$
\begin{aligned}
\mathrm{C}_{T}(a) & =\langle\pi, b\rangle, \Omega_{1}\left(\mathrm{C}_{T}(a)\right)=\Omega_{1}\left(\left\langle\pi^{2}, b\right\rangle\right)=W ; \\
\mathrm{C}_{T}\left(a b^{2}\right) & =\left\langle\pi^{2}, b, a \pi\right\rangle \geq\left\langle a \pi, \pi^{2}\right\rangle \simeq D_{16^{\circ}}
\end{aligned}
$$

Hence $\mathbf{C}_{T}\left(a b^{2}\right)$ and $\mathbf{C}_{T}(a)$ are not isomorphic; but they are Sylow 2-subgroups of $\mathrm{C}_{G}\left(a b^{2}\right)$ and $\mathbf{C}_{G}(a)$, so would be isomorphic if $a b^{2} \sim a$.

If $x \in b \Phi(T)$, then $x^{2}=b^{2}, b^{2} a^{2}, b^{2} a$, or $b^{2} a^{-1}$. But (as just shown) none of these are conjugate to elements of $\left\langle\pi^{2}\right\rangle$.

We will now discuss the case $C_{T}(V) \cong Z_{2^{n}} \times Z_{4}$, where $n \geq 3$; this case closely parallels the case $\mathbf{C}_{T}(V)=V$.

Let $C=\mathrm{C}_{T}(V)=\langle a, b\rangle$ where $\omega=a^{2^{n-1}} \in \mathrm{Z}(T)$, and $b$ has order 4. We take $\left\{a^{2^{n-2}}, b\right\}$ as a basis for $V$; then the matrix-group induced on $V$ by $T$ is contained in $\left\langle B^{+},\left(\begin{array}{ll}1 & 0 \\ 1 & 1\end{array}\right)\right\rangle$, and $\left(\begin{array}{ll}1 & 0 \\ 2 & 1\end{array}\right)$ is central.

(i) If $y \in T$ induces $\left(\begin{array}{ll}1 & 0 \\ 2 & 1\end{array}\right)$ on $V$, then we may replace $a$ by $a b$ if necessary to get $a^{y}=a, b^{y}=b$, and $y^{2} \in a \Phi(C)$. (Note that if $a$ is replaced by $a b$ (and $b$ by $b)$, we still have $a^{2^{n-1}}=\omega$ and $A_{T}(V) \leq\left\langle B^{+},\left(\begin{array}{ll}1 & 0 \\ 1 & 1\end{array}\right)\right\rangle$.)

Proof. Since $y$ induces $\left(\begin{array}{ll}1 & 0 \\ 2 & 1\end{array}\right)$ on $V$, we have $a^{y}=a^{i} b^{j}$ where $i \equiv 1 \bmod 4$; and $b^{y}=b . y^{2} \in C$ which is Abelian, so $y^{2}$ centralizes $a$, and

$$
a=\left(a^{i} b^{j}\right)^{i}(\omega b)^{j}=a^{i^{2}} \omega^{j} b^{j(i+1)} .
$$

But $i+1 \equiv 2 \bmod 4$, so $j$ must be even; so we have $a=a^{i^{2}}$, so $i=1$ or $1+2^{n-1}$, and $a^{y}=a z$ for some $z \in W$.

Suppose there is $y$ inducing $\left(\begin{array}{ll}1 & 0 \\ 2 & 1\end{array}\right)$ on $V$ with $y^{2}=1$. Then for any $t \in T$, $[y, t] \in C$ and $y$ inverts $[y, t]$; but the only elements of $C$ which $y$ inverts, are in $W$, so $[y, T] \leq W$ and $\langle y, W\rangle$ is a normal $E_{8}$ of $T$.

Hence $y C$ contains no involutions. $z \in\langle\omega\rangle$, for otherwise $C_{C}(y)=\left\langle a^{2}, b^{2}\right\rangle=$ coboundaries of $y$ on $C$, so $y C$ would contain an involution. (i) follows.

(ii) $T_{0}$ contains $\alpha \sim \omega$ inducing $\left(\begin{array}{ll}3 & 0 \\ 0 & 1\end{array}\right)$ on $V$. If $\phi \in T-T_{0}$, then $a^{\phi}$ induces $\left(\begin{array}{ll}3 & 0 \\ 2 & 1\end{array}\right)$, and $\left(a a^{\phi}\right)^{2} \in a \Phi(C)$. 
Proof. Same as the proof of (ii) in the case $\mathrm{C}_{T}(V)=V$.

(iii) No element of $T_{0}$ inverts $V$. Hence $A_{T_{0}}(V)=\left\langle\left(\begin{array}{ll}3 & 0 \\ 0 & 1\end{array}\right),\left(\begin{array}{ll}1 & 0 \\ 2 & 1\end{array}\right)\right\rangle$ (for if larger, it contains $\left(\begin{array}{ll}3 & 0 \\ 0 & 3\end{array}\right)$ since it is invariant under $\left.\left(\begin{array}{ll}1 & 0 \\ 1 & 1\end{array}\right)\right)$.

Proof. Suppose $\delta \in T_{0}$ inverts $V . a^{\delta}=a s$ for some $s \in C$. Let $\phi \in T-$ $T_{0}$; then $\delta=\delta^{\phi} x$ for some $x \in C$, and $(a s)^{\phi}=\left(a^{\delta}\right)^{\phi}=\left(a^{\phi}\right)^{\delta \phi}$. Hence

$$
\left(a^{\phi}\right)^{\delta}=\left(a^{\phi}\right)^{\delta^{\phi} x}=(a s)^{\phi x}=a^{\phi} s^{\phi}\left[a^{\phi}, x\right] \text {. }
$$

Now

$$
\left(\left(a a^{\phi}\right)^{2}\right)^{\delta}=\left(a s \cdot a^{\phi} s^{\phi}\left[a^{\phi}, x\right]\right)^{2}=\left(a \alpha^{\phi} s\left[s, a^{\phi}\right] s^{\phi}\left[\alpha^{\phi}, x\right]\right)^{2} .
$$

But $a a^{\phi}$ induces $\left(\begin{array}{ll}1 & 0 \\ 2 & 1\end{array}\right)$ on $V$, so by (i), $\left[C, \alpha a^{\phi}\right] \leq W$, and

$$
\begin{aligned}
\left(\left(a a^{\phi}\right)^{2}\right)^{\delta} & \equiv\left(a \alpha^{\phi}\right)^{2}\left(s\left[s, \alpha^{\phi}\right] s^{\phi}\left[\alpha^{\phi}, x\right]\right)^{2} \bmod w \\
& \equiv\left(a \alpha^{\phi}\right)^{2}\left(s s^{\phi}\right)^{2}\left[s, a^{\phi}\right]^{2}\left[a^{\phi}, x\right]^{2} \cdot \bmod W .
\end{aligned}
$$

Write. $y=\left(a \alpha^{\phi}\right)^{2}$, so that $C=\langle\gamma, b\rangle$ by (i); then

$$
y^{\delta} \equiv \gamma\left(s s^{\phi}\right)^{2}\left[s, a^{\phi}\right]^{2}\left[\alpha^{\phi}, x\right]^{2} \bmod w .
$$

Now $C_{1}=\left\langle\gamma^{2}, b\right\rangle$ is a characteristic maximal subgroup of $C$, so $\left[s, a^{\phi}\right]$, ss ${ }^{\phi}$, and $\left[a^{\phi}, x\right]$ all lie in $C_{1}$. Hence

$$
\gamma^{\delta} \equiv \gamma \quad \bmod \left\langle C_{1}^{2}, w\right\rangle=\left\langle\gamma^{4}, b^{2}\right\rangle .
$$

However, computing $\gamma^{\delta}$ directly, we have $\gamma^{\delta}=\gamma^{-1} c$, for some $c \in C ; \delta^{2} \epsilon$ $C$ implies that $\delta^{2}$ centralizes $\gamma$, so

$$
\gamma=\left(\gamma^{-1} c\right)^{\delta}=\left(\gamma^{-1} c\right)^{-1} c^{\delta}=\gamma c^{-1} c^{\delta},
$$

so that $\delta$ centralizes $c$ and therefore $c \in W$; so $\gamma^{\delta} \equiv \gamma^{-1} \bmod \left\langle\gamma^{4}, b^{2}\right\rangle$, contradicting the above.

We have all this for any $\phi \in T-T_{0}$. If $T-T_{0}$ contains an involution, we shall choose $\phi$ to be an involution.

(iv) Suppose $T-T_{0}$ contains an involution $\phi$. Then, letting $\pi=a \phi$, we have

$T=\langle a, \pi, b: a$ inverts $\pi$ and centralizes $b ;$

$$
\left.a^{2}=\pi^{2^{n+2}}=b^{4}=1 ; \pi^{b}=\pi^{1+2^{n}}\right\rangle \quad(n \geq 3) .
$$

Proof. It follows from (ii) that $\pi$ has order $2^{n+2}$, and that $C=\left\langle\pi^{4}, b\right\rangle$. We need to compute $\pi^{b} ; \pi^{b}=(a \phi)^{b}=a \phi^{b}$. 
Now $b^{\phi}=a^{2^{n-2} i} b^{j}$ for some odd $i$ and $j$, since $V / W$ is operator-isomorphic to $W$. (We have taken $a=\pi^{4}$; this is consistent with (i).) Then

$$
b=b^{\phi^{2}}=\left(a^{2^{n-2} i} b^{j}\right)^{\phi}=a^{-2^{n-2} i}\left(a^{2^{n-2} i} b^{j}\right)^{j}=a^{2^{n-2} i(j-1)} b^{j^{2}} ;
$$

so $j=1$, and $b^{\phi}=a^{2^{n-2} i} b$ for odd $i$. Then

$$
\pi^{b}=a \phi a^{-2^{n-2} i}=\pi \pi^{-2^{n} i}
$$

If $i \equiv-1 \bmod 4$, we are done. If $i \equiv 1 \bmod 4$, then the involution $a \phi \alpha$ of $T-$ $T_{0}$ has

$$
b^{\alpha \phi \alpha}=b^{\phi a}=\left(a^{2^{n-2} i} b\right)^{a}=a^{-2^{n-2} i} b,
$$

so using $\phi^{a}$ instead of $\phi$ (which replaces $\pi=a \phi$ by $a \phi^{a}=a \phi[\phi, a]=\pi \pi^{-2}$ which is a power of $\pi$ ), we may assume $\pi^{b}=\pi^{1+2^{n}}$, as claimed.

We will now use a transfer argument similar to the one where $C=V$. Again, $\Phi(T)=\left\langle\pi^{2}, b^{2}\right\rangle$, and $\{1, b, \pi, \pi b, a, a b, a \pi, a \pi b\}$ is a transversal to $\Phi(T)$ in $T$. $T^{\prime}=\left\langle\pi^{2}\right\rangle$, and by Grü's theorem, $T$ is generated by $G$-conjugates of elements of $\left\langle\pi^{2}\right\rangle$.

The elements of $\pi \Phi(T) \cup \pi b \Phi(T)$ have order $2^{n+2}$, so are not conjugate to elements of $\left\langle\pi^{2}\right\rangle$.

If $x \in a b \Phi(T)$, then $x^{2}=b^{2}$ or $b^{2} \omega=\omega_{1}$ or $\omega_{2}$. So if $x \sim z \in\left\langle\pi^{2}\right\rangle$, then $x^{2} \sim z^{2}$ gives $\omega_{1} \sim \omega$, contrary to hypothesis.

If $x \in b \Phi(T)$, then $x^{2}=b^{2} \pi^{4 k}$ for some $k$. So if $x \sim z \in\left\langle\pi^{2}\right\rangle$, then $\left\langle b^{2} \pi^{4 k}\right\rangle$ $\sim\left\langle\pi^{4 k}\right\rangle$. But $\mathrm{C}_{T}\left(\pi^{4 k}\right)=\langle\pi, b\rangle$ is a Sylow 2-subgroup of $\mathrm{C}_{G}\left(\pi^{4 k}\right)$, while $\mathrm{C}_{T}\left(b^{2} \pi^{4 k}\right) \geq$ $\left\langle\pi^{2}, b\right\rangle$. So there is $g \in G$ with

$$
\left\langle b^{2} \pi^{4 k}\right\rangle^{8}=\left\langle\pi^{4 k}\right\rangle, \quad\left\langle\pi^{2}, b\right\rangle^{8} \leq\langle\pi, b\rangle,
$$

and hence $\left\langle\pi^{2}, b\right\rangle^{8}=\left\langle\pi^{2}, b\right\rangle$, i.e., $g \in \mathbf{N}_{G}\left(\left\langle\pi^{2}, b\right\rangle\right)$. However, as $\left\langle\pi^{2}, b\right\rangle$ does not admit in $G$ any automorphisms of odd order, $T$ covers $\mathbf{N}_{G}\left(\left\langle\pi^{2}, b\right\rangle\right)$; and $\left\langle b^{2} \pi^{4 k}\right\rangle$ is not $T$-conjugate to $\left\langle\pi^{4 k}\right\rangle$.

If $x \in a \pi b \Phi(T)$, then $x^{2}=b^{2} \pi^{ \pm 2^{n}}$, and by the above, $x$ is not conjugate to any element of $\left\langle\pi^{2}\right\rangle$.

We now have five nonidentity cosets of $\Phi(T)$ in $T$ which cannot contain conjugates of $\left\langle\pi^{2}\right\rangle$, which violates Grün's theorem.

We now assume that $T-T_{0}$ contains no involutions. Let $\phi$ be any element of $T-T_{0}$ which induces an automorphism of $V$ whose square is 1 . If $\phi^{2}$ may be taken in $V$, then $\phi V$ contains an involution; hence, $\langle\phi, C\rangle / V$ contains only one involution. We shall show that if $[\langle\phi, C\rangle: V]\rangle 8$, then $\langle\phi, C\rangle / V$ is non- 
Abelian. Let $y=\alpha \alpha^{\phi}$; then (i) implies that $C=\left\langle y^{2}, b\right\rangle$, and if $[\langle\phi, C\rangle: V] \geq 8$ then $y^{2}$ has order $\geq 4 \bmod V$. Now

$$
y^{\phi}=\left(\alpha \alpha^{\phi}\right)^{\phi}=y^{-1}\left[\alpha, \phi^{2}\right] \text {. }
$$

$\phi^{2} \in C$, so $\phi^{2}=b^{s} y^{2 t}$ for some $s, t$, and $\left[a, \phi^{2}\right]=\left[a, y^{2 t}\right]=y^{4 t} ; y^{\phi}=y^{-1+4 t}$. Since $y^{2}$ has order $\geq 4 \bmod V$, it follows that $\langle\phi, C\rangle / V$ is non-Abelian. Therefore, $\langle\phi, C\rangle / V$ is either cyclic of order 4 , or generalized quaternion, with $\phi^{2} \equiv$ $a^{2^{n-3}} \bmod V$.

Let $y=\alpha \alpha^{\phi}, a=y^{2}$ (this is consistent with (i)). Then

$$
\begin{aligned}
y^{\phi} & =\left(a \alpha^{\phi}\right)^{\phi}=y^{-1}\left[\alpha, \phi^{2}\right]=y^{-1}\left[\alpha, a^{2^{n-3} i} b^{q}\right], \text { odd } i \\
& =y^{-1} a^{2^{n-2} i}=y^{-1+2^{n-1} i} ; \\
a^{\phi} & =a^{-1} \omega ; \quad\left(a^{2^{n-2}}\right)^{\phi}=a^{-2^{n-2}},
\end{aligned}
$$

where $\omega=a^{2^{n-1}}$.

Since $V / W$ is operator-isomorphic to $W$, we have $b^{\phi}=a^{2 n-2 k} b^{l}$ for some odd $k$ and $l$; since $\phi^{2}$ centralizes $b$, it follows as in the case $\phi^{2}=1$ that $l=$ 1. Then $\mathrm{C}_{V}(\phi)=\left\langle a^{2^{n-2}} b^{2}\right\rangle$, and so $\phi^{2} \equiv a^{2^{n-3} j} b \bmod W$, for some odd $j$. Replace $\phi$ by $\phi^{-1}$ if necessary, to get $\phi^{2}=a^{2 n-3 j} b$, for some odd $j$. Since $\phi$ centralizes $\phi^{2}$, we get

$$
a^{2^{n-3}} b=a^{-2^{n-3} j} \omega^{2^{n-3}}\left(a^{2^{n-2} k} b\right)
$$

so that $b^{\phi}=a^{2^{n-2} j} \omega^{2^{n-3}} b$.

Therefore,

$$
\begin{array}{r}
T=\left\langle a, b, y, \alpha, \phi:|a|=2^{n} \text { and }\langle a, b\rangle \cong \mathbf{Z}_{2^{n}} \times \mathbf{Z}_{4} ; y^{2}=a, b^{y}=\omega b ; \alpha^{2}=1,\right. \\
y^{a}=y^{-1}, b^{a}=b ; \phi^{2}=a^{2^{n-3}} j_{b}, y^{\phi}=y^{-1+2^{n-1} i}, \\
b^{\phi}=a^{\left.2^{n-2} j \omega^{2^{n-3}} b, \alpha^{\phi}=a y\right\rangle .}
\end{array}
$$

Here $\omega=a^{2^{n-1}}$ and $i, j$ are odd.

$T$ is generated by $a$ and $\phi$, and $\Phi(T)=\langle y, b\rangle . T^{\prime}=\langle y\rangle$. We shall use the same transfer argument. We take $\{1, \phi, a, a \phi\}$ as a transversal to $\Phi(T)$ in $T$.

The elements of $a \phi \Phi(T)$ have order $2^{n+2}$, so cannot be conjugate to any element of $\langle y\rangle$.

Let $x \in \phi \Phi(T)$; then $x^{2}=a^{2^{n-3} k} b^{l}$ for some odd $k$ and $l$. So if $x \sim z \epsilon$ $\langle y\rangle$, then there are odd $k, m$ such that $a^{2^{n-3} m} \sim a^{2^{n-3} k} b$. Now $a^{2^{n-3} k} b$ is conjugated by $y$ to its own fifth power, and by $a$ to an element which generates 
a different cyclic group of order 8 in $T$; so $\left[T: \mathrm{C}_{T}\left(a^{2^{n-3 k}} b\right)\right] \geq 4$. Also, $\mathrm{C}_{T}\left(a^{2^{n-3} m}\right)=\langle y, b, a \phi\rangle$ if $n \geq 4$, and $\langle y, b\rangle$ if $n=3$. Therefore, $\mathbf{C}_{T}\left(a^{2^{n-3} m}\right)$ is a Sylow 2-subgroup of $C_{G}\left(a^{2^{n-3}} m\right)$, and there is $g \in G$ with

$$
\left(a^{2^{n-3} k} b\right)^{8}=a^{2^{n-3} m}, \quad\langle a, b\rangle^{8} \leq \mathrm{C}_{T}\left(a^{2^{n-3} m}\right) .
$$

It follows that $\langle a, b\rangle^{8}=\langle a, b\rangle$. But $T$ covers $\mathbf{N}_{G}(\langle a, b\rangle)$, and $a^{2^{n-3} m}$ is not $T$ conjugate to $a^{2^{n-3} k} b$.

Hence, two of the three nonidentity cosets of $\Phi(T)$ in $T$ are free of conjugates of elements of $T^{\prime}$. Hence $T$ is not generated by conjugates of elements of $T^{\prime}$, and this case is finished.

We may now assume that $\Omega_{3}\left(C_{T}(V)\right)$ is a group of order 64 and exponent 8 . In this case it is quite easy to find the group induced on $V$ by $T / C$ (where $C=$ $\left.\mathrm{C}_{T}(V)\right)$. Let $c, d$ generate $V_{3}=\Omega_{3}(C)$ so that $c^{4}=\omega$, and take $\left\{c^{2}, d^{2}\right\}$ as a basis for $V$. Then the matrix-group induced on $V$ by $T$ is a subgroup of $\left\langle\left(\begin{array}{ll}1 & 0 \\ 1 & 1\end{array}\right)\right.$, $\left.B^{+}\right\rangle$, and $\left(\begin{array}{ll}1 & 0 \\ 2 & 1\end{array}\right)$ is central, as before.

Let $\cong$ be any automorphism of $V_{3}$ which fixes $W$ elementwise. It is easily checked that if $c^{D}=c^{i} d^{j}$ and $d^{D}=c^{k} d^{l}$, then (though $V_{3}$ need not be Abelian)

$$
c^{D^{2}}=c^{i^{2}+j k} d^{j(i+l)}, \quad d^{D^{2}}=c^{k(i+l)} d^{j k+l^{2}} ;
$$

and if $\supset$ has the matrix $\left(\begin{array}{ll}a & \beta \\ y & \delta\end{array}\right)$ on $V$ with respect to $\left\{c^{2}, d^{2}\right\}$, then

$$
\left(\begin{array}{ll}
i & j \\
k & l
\end{array}\right) \equiv\left(\begin{array}{ll}
a & \beta \\
\gamma & \delta
\end{array}\right) \bmod 4
$$

(i) If $y \in T$ induces $\left(\begin{array}{ll}1 & 0 \\ 2 & 1\end{array}\right)$ on $V$, then $y^{2}$ induces $\left(\begin{array}{ll}1 & 0 \\ 4 & 1\end{array}\right)$ on $V_{3}$, i.e., $y^{-2} d y=d$. (This implies $d$ is not central in $C_{\text {. }}$ )

Proof. Let $y$ have matrix $\left(\begin{array}{cc}i & j \\ k & l\end{array}\right)$ on $V_{3}$, so $\left(\begin{array}{cc}i & j \\ k & l\end{array}\right) \equiv\left(\begin{array}{ll}1 & 0 \\ 2 & 1\end{array}\right) \bmod 4$. Then $y^{2}$ has the matrix

$$
\left(\begin{array}{cc}
i^{2}+j k & j(i+l) \\
k(i+l) & j k+l^{2}
\end{array}\right) \equiv\left(\begin{array}{ll}
1 & 0 \\
4 & 1
\end{array}\right) \bmod 8
$$

(ii) None of the matrices $\left(\begin{array}{ll}3 & 0 \\ 2 & 3\end{array}\right),\left(\begin{array}{ll}3 & 2 \\ 0 & 3\end{array}\right),\left(\begin{array}{ll}1 & 2 \\ 2 & 1\end{array}\right)$ can be induced on $V$ by $T$.

Proof. If $x \in T$ induces one of the above on $V$, then $x^{2}$ does not centralize $V_{3}$. But $x^{2} \in C \cap \mathrm{C}_{T}(x) ; \Omega_{2}(C)=V$, and $\mathrm{C}_{V}(x)=W$, so $C \cap \mathrm{C}_{T}(x)=W$ and $x^{2}$ must centralize $V_{3}$.

(iii) $T_{0}$ induces precisely $\left\langle\left(\begin{array}{ll}3 & 0 \\ 0 & 1\end{array}\right),\left(\begin{array}{ll}1 & 0 \\ 2 & 1\end{array}\right)\right\rangle$ on $V$, and $T_{0}$ contains $a \sim \omega$ inducing $\left(\begin{array}{ll}3 & 0 \\ 0 & 1\end{array}\right)$.

Proof. The only automorphisms possible for $\alpha$ with $W(\alpha ; G)=\langle\omega\rangle$ are 
$\left(\begin{array}{ll}3 & 0 \\ 0 & 1\end{array}\right),\left(\begin{array}{ll}3 & 0 \\ 2 & 1\end{array}\right)$, and $\left(\begin{array}{ll}1 & 0 \\ 2 & 1\end{array}\right)$. Since $\left(\begin{array}{ll}1 & 0 \\ 2 & 1\end{array}\right)$ is not induced by an involution, and $\left(\begin{array}{ll}1 & 0 \\ 1 & 1\end{array}\right)$ conjugates $\left(\begin{array}{ll}3 & 0 \\ 0 & 1\end{array}\right)$ to $\left(\begin{array}{ll}3 & 0 \\ 2 & 1\end{array}\right)$, a exists as claimed. If $T_{0}$ induced more than $\left\langle\left(\begin{array}{ll}3 & 0 \\ 0 & 1\end{array}\right)\right.$, $\left.\left(\begin{array}{ll}1 & 0 \\ 2 & 1\end{array}\right)\right\rangle$, it would induce one of the matrices in (ii) (which are the nonidentity elements of a four-group complementary in $B^{+}$to $\left.\left\langle\left(\begin{array}{ll}3 & 0 \\ 0 & 1\end{array}\right),\left(\begin{array}{ll}1 & 0 \\ 2 & 1\end{array}\right)\right\rangle\right)$.

We will now determine the structure of $C$. Let $r$ be the largest integer with $\left[V_{r}: V_{r-1}\right]=4$, where $V_{i}=\Omega_{i}(C)$. If $C>V_{r}$, then let $x$ generate $C \bmod V_{r}$; $\Omega_{1}(\langle x\rangle)=\langle\omega\rangle$ and we can take $z \in V_{r}$, so that $V_{r}=\left\langle x^{2^{n-r}}, z\right\rangle$, where $C$ has exponent $2^{n}$. If $C=V_{r}$, then take $x \in V_{r}$, with $x^{2^{r-1}}=\omega$, and then take $z \in V_{r}$ so that $C=V_{r}=\langle x, z\rangle$.

(iv) $C / V_{r-2}$ is Abelian, but $C / V_{r-3}$ is non-Abelian with a derived group $D / V_{r-3}$ of order $2 ; D^{2 r-3}=\langle\omega\rangle$. If $C>V_{r}$, then $D / V_{r-3}$ is generated by the only involution that is an eighth power in $\mathrm{C} / \mathrm{V}_{r-3}$.

Proof. With the above notation in $C, C^{\prime}$ is generated by $[x, z]$ since $C$ is metacyclic. Now $\left\{x^{2^{n-3}}, z^{2^{n-3}}\right\}$ is a basis for $V_{3}$ as in (i). It follows from Lemma $6(c)$ that $\Phi(C)=C^{2}$ centralizes $V_{3}$. Hence $\langle\Phi(C), z\rangle$ centralizes $z^{2^{r-3}}$, and by $(i),\left[x, z^{2^{r-3}}\right]=\omega$ has order 2 . By Lemma $6(c),[x, z]$ then has order $2^{r-2}$ so lies in $V_{r-2}-V_{r-3}$.

Let $\phi$ be any element of $T-T_{0}$. The action of $\phi$ on $W$ shows that $\omega$ is the only characteristic involution of $C$. Now $V_{r-2} / V_{r-3}$ has only one involution which is characteristic in $C / V_{r-3}$, and this must map to $\omega$ under the isomorphism of Lemma $6(f)$. This completes the proof of (iv).

Now take $\alpha$ as in (iii), and take any $\phi \in T-T_{0}$ which induces an automorphism of $V$ whose square is 1 . Let

$$
y=\alpha \alpha^{\phi} ; \quad a=y^{2} .
$$

By (i), $y^{2}$ does not centralize $d$ (for $d$ as in (i)). Since $\Phi(C)$ centralizes $V_{3}$, it follows that $C=\left\langle y^{2}, e\right\rangle$ where $e \in C-\Phi(C)$ has some power equal to $d$.

If $y^{2}$ were not of maximal order in $C$, then $e$ must be of maximal order in $C$, and $\Omega_{1}(\langle e\rangle)=\langle\omega\rangle$; but we already know that $\Omega_{1}(\langle d\rangle) \neq\langle\omega\rangle$. Hence $y^{2}$ has maximal order in $C$, and $C=\left\langle y^{2}, V_{r}\right\rangle$. (Here $r$ is the largest integer with $\left[V_{r}: V_{r-1}\right]$ $=4$, and $2^{n}$ is the exponent of $C$ and hence the order of $y^{2}$.)

(v) Taking $\phi, \alpha, y, a$ as above, let $P=\langle y, C\rangle$ (so $P$ is a normal subgroup of index 4 in $T)$. Then $P=\langle y, b\rangle$ where $a$ inverts $y$ and centralizes $b$, and $|b|=2^{r}$, and $\langle b\rangle \cap\langle y\rangle=1$.

Proof. For any $z, t \in P,[\alpha, z t]=[\alpha, t][\alpha, z] t$, so $[\alpha, z t]=[\alpha, t]$ if and only if $z \in \mathbf{C}_{G}(a)$. Hence

$$
|\{[a, z]: z \in P\}|=|P| /\left|\mathbf{C}_{P}(a)\right| \text {. }
$$


Suppose we knew $\left|\mathrm{C}_{p}(\alpha)\right|=2^{r+1}$, or equivalently, $[P, a]=\left\langle y^{2}\right\rangle$. Now $\mathbf{C}_{V}(a)=\left\langle\omega, d^{2}\right\rangle$, and so $\mathbf{C}_{p}(\alpha) / W$ is cyclic of order $2^{r-1}$. Moreover, $\omega$ is not a square in $\mathbf{C}_{p}(\alpha)$, since it is not a square in $\mathbf{C}_{V}(\alpha)$. It follows that $\mathbf{C}_{p}(a)=$ $\langle W, b\rangle$ for some $b$, and $b^{2^{r-1}}=d^{4}$ or $\omega d^{4}$ since otherwise $C_{P}(a)$ would contain an $E_{8}$ (while $P$ contains no $E_{8}$ ). This would establish (v).

We first show that $[P, a] \leq\left\langle y^{2}, W\right\rangle=\langle a, W\rangle$. Namely, let $C_{i}=\left\langle y, \Omega_{i}(C)\right\rangle$; then if $[P, a] \notin\langle a, W\rangle$, there is $i$ with $\left[C_{i}, a\right] \leq\langle a, W\rangle$ but $\left[C_{i+1}, a\right] \notin\langle a, W\rangle$. Let $z \in C_{i+1}$ with $[z, a] \notin\langle a, W\rangle$. $\alpha$ inverts its commutator $[a, z]$, so $[[a, z], \alpha]$ $=[a, z]^{-2}$. But $\left[C_{i+1}: C_{i}\right]=2$, and $C_{i}$ and $C_{i+1}$ are $\alpha$-invariant, so $[a, z] \epsilon$ $C_{i}$, and hence $[[\alpha, z], \alpha] \in\langle a, W\rangle$. So $[\alpha, z]^{2} \in\langle a, W\rangle$ but $[\alpha, z] \notin\langle a, W\rangle$.

This implies (Lemma $6(\mathrm{~g})$ ) that

$$
[a, z]=a^{j} d^{2 k} \text { for some } j \text { and some odd } k \text {. }
$$

But then

$$
[a, z]^{\alpha}=\left(a^{j} d^{2 k}\right)^{\alpha}=a^{-j} d^{2 k} \neq[a, z]^{-1} .
$$

Now $[P, a] \leq\left\langle y^{2}, W\right\rangle$ implies $\left|\mathbf{C}_{P}(a)\right| \geq 2^{r}$. If $\left|\mathbf{C}_{P}(a)\right|=2^{r}$, then the above argument gives $\mathbf{C}_{P}(a) \cong \mathbf{Z}_{2} \times \mathbf{Z}_{2^{r-1}}$, and there is $b \in P$ with $b^{2} \in \mathbf{C}_{P}(a)$ and $|b|=2^{r}$. Then

$$
b^{2}=\left(b^{2}\right)^{a}=\left(b^{a}\right)^{2}=(b[b, a])^{2}=b^{2}[b, a]^{b}[b, a] .
$$

Thus $b$ inverts $[b, a]$, so $[b, a] \in W$ as $\Omega_{2}(C)$ is central in $C$. But $W(a ; G)=$ $\langle\omega\rangle$, so $[b, a] \epsilon\langle\omega\rangle$, and $b$ or $b c^{2}$ (for $c$ as in (i)) lies in $C_{p}(a)$. Hence $\left|\mathbf{C}_{P}(a)\right|=2^{r+1}$, and (v) holds.

(v) is true for any $\phi \in T-T_{0}$ which induces an automorphism of $V$ whose square is 1 . We shall now determine $T$ if $\phi$ can be taken as an involution.

(vi) Suppose $T-T_{0}$ contains an involution $\phi$. Let $\pi=a \phi$, so that $y=$ $\pi^{2}$ and $a=\pi^{4}$. Then

$T=\langle a, \pi, b: a$ inverts $\pi$ and centralizes $b ;$

$$
\left.a^{2}=\pi^{2^{n+2}}=b^{2^{r}}=1 ; \pi^{b}=\pi^{1+2^{n-r+2} i}\right), \text { for some odd } i \text {. }
$$

Proof. $b^{\phi}=b^{j} a^{2^{n-r} k}$ for odd $j$ and $k$, since $V_{r} / V_{r-1}$ is operator-isomorphic to $W$. Since $\phi^{2}=1$, and $\phi$ inverts $\pi$,

$$
b=b^{\phi^{2}}=\left(b^{j} a^{2^{n-r} k}\right)^{\phi}=\left(b^{j} a^{2^{n-r} k}\right)^{j} a^{-2^{n-r} k}=b^{j^{2}} a^{2^{n-r} k j k_{a}-2^{n-r} k},
$$

since $\langle a\rangle=[P, a] \triangleleft\langle P, a\rangle$ and hence $\langle a\rangle$ is a normal cyclic subgroup of the metacyclic group $C$; as $a$ is also a generator of $C$, this gives $C^{\prime} \leq\langle a\rangle$. Also, 
$\Omega_{2}(C) \leq \mathrm{Z}(C)$ implies that $K \equiv 1 \bmod 4$. It follows that $j \equiv 1 \bmod 4$, and as $j^{2} \equiv$ $1 \bmod 2^{r}$, we have $j=1$ or $j=1+2^{r-1}$. If $j=1+2^{r-1}$, then

$$
b=b^{\phi^{2}}=\left(b^{1+2^{r-1}} a^{2^{n-r} k}\right)^{\phi}=\left(b^{1+2^{r-1}} a^{2^{n-r} k}\right)\left(b^{1+2^{r-1}} a^{2^{n-r} k}\right)^{2^{r-1}} a^{-2^{n-r} k} \text {. }
$$

Now by Lemma $6(f),\left(b^{1+2^{r-1}} a^{2^{n-r} k}\right)^{2^{r-1}}=b^{2^{r-1}} a^{2^{n-1} k}=b^{2^{r-1}} \omega$. Hence

$$
b=b^{1+2^{r-1}} a^{2^{n-r} k} b^{2^{r-1}} \omega a^{-2^{n-r} k}=b \omega,
$$

a contradiction. Therefore $b^{\phi}=b a^{2^{n-\tau} k}$, for odd $k$.

Hence,

$$
\pi^{b}=(a \phi)^{b}=a b^{-1} \phi b=a \phi\left(b^{-1}\right)^{\phi} b=a \phi a^{-2^{n-r} k} b^{-1} b=\pi^{1-2^{n-r+2} k},
$$

and (vi) is proved.

By Grün's theorem, $T$ is generated by conjugates of elements of $T^{\prime}=\langle y\rangle$. $\Phi(T)=\left\langle y, b^{2}\right\rangle$.

The elements of $\pi \Phi(T) \cup \pi b \Phi(T)$ have order $2^{n+2}$, so are not conjugate to elements of $\langle y\rangle$.

If $x \in a b \Phi(T)$, then $x=a b^{j} y^{k}$ for some odd $j$, and

$$
x^{2}=b^{j} y^{-k} b^{j} y^{k}=b^{2 j} y^{-k\left(1+2^{n-r+2} i\right)} y^{k}=b^{2 j_{a} a^{-2^{n-r+1}} i k} ; x^{2^{r-1}}=\omega_{1} \text { or } \omega_{2} \text {. }
$$

So $x \sim z \epsilon\langle y\rangle$ would imply $\omega_{1} \sim \omega$, contrary to hypothesis.

If $x \in b \Phi(T)$, then $x=b^{j} y^{k}$ for odd $j$, and

$$
x^{2}=b^{2 j} y^{2 m}, \text { for some } m ; \quad x^{2^{r-1}}=b^{2^{r-1}} a^{2^{r-2} m} .
$$

So if $x \sim z \in\langle y\rangle$, then $\left\langle\omega_{1} a^{2^{r-2} m}\right\rangle \sim\left\langle a^{2^{r-2} m}\right\rangle$ for some m. Now $\mathbf{C}_{T}\left(a^{2 r-2 m}\right)=$ $\langle\pi, b\rangle$ has index 2 in $T$, so is a Sylow 2-subgroup of $\mathbf{C}_{\boldsymbol{g}}\left(a^{2^{r-2} m}\right)$. So there is $g \in G$ with

$$
\left\langle\omega_{1} a^{2^{r-2} m}\right\rangle^{g}=\left\langle a^{2^{r-2} m}\right\rangle,\langle y, b\rangle^{8} \leq\langle\pi, b\rangle,
$$

and hence $\langle y, b\rangle^{g}=\langle y, b\rangle$. But $T$ covers $\mathbf{N}_{g}(\langle y, b\rangle)$, and $\left\langle\omega_{1} a^{2^{r-2} m}\right\rangle$ is not $T$ conjugate to $\left\langle a^{2^{r-2} m}\right\rangle$.

If $x \in a \pi b \Phi(T)=\phi b \Phi(T)$, then $x=\phi b^{j} y^{k}$ for some odd $j$, and

$$
\begin{aligned}
x^{2} & =\left(b^{\phi}\right)^{j}\left(y^{\phi}\right)^{k} b^{j} y^{k}=b^{2 j} a^{2^{n-r} m} \text { for odd } m ; \\
x^{2^{r-1}} & =b^{2^{r-1}} a^{ \pm 2^{n-2}},
\end{aligned}
$$

and by the above, $x$ is not conjugate to any element of $\langle y\rangle$ 
We now have five nonidentity cosets of $\Phi(T)$ in $T$ which cannot contain $G$ conjugates of elements of $T^{\prime}$, violating Grün's theorem.

(vii) Suppose $T-T_{0}$ contains no involutions. Let $\phi$ be any element of $T$ $-T_{0}$ which induces on $V$ an automorphism whose square is 1 . Then $\langle\phi, C\rangle / V_{\text {, }}$ is either cyclic of order 4 or generalized quaternion, with $\phi^{2} \equiv a^{2^{n-r-1}} \bmod V_{r}$.

Proof. If $\phi^{2} \in V_{r}$, then $\left\langle\phi, V_{r}\right\rangle / V_{r-1} \cong D_{8}$, and there is $v \in V_{r}$ with $(\phi v)^{2}$ $\epsilon V_{r-1} ;\left\langle\phi v, V_{r-1}\right\rangle / V_{r-2} \cong D_{8}$ and iteration gives $x \in V_{r}$ with $(\phi x)^{2}=1$. So $\phi^{2} \notin V_{r}$, and $\langle\phi, C\rangle / V_{r}$ contains only one involution. We need to show that $\langle\phi, C\rangle / V_{r}$ is non-Abelian if its order is $\geq 8$. We have $y=\alpha \alpha^{\phi}$, and so $y^{\phi}=$ $y^{-1}\left[a, \phi^{2}\right]$; but by (v), $C=\left\langle y^{2}, b\right\rangle$, and $\phi^{2} \in C$, so $\left[a, \phi^{2}\right]=\left[a, y^{2 t}\right]=y^{4 t}$ for some $t$, and $y^{\phi}=y^{-1+4 t}$. Since $y^{2}$ has order $\geq 4 \bmod V_{r}$, this gives that $\langle\phi, C\rangle / V_{r}$ is non-Abelian; (vii) follows.

(viii) Suppose $T-T_{0}$ contains no involutions. Let $\phi$ be any element of $T-T_{0}$ which induces on $V$ an automorphism whose square is 1 . Take $a, y, a$ as in (v). Then $T=\langle a, b, a, y, \phi\rangle=\langle\langle y, b\rangle, a, \phi\rangle$, where

$$
\begin{aligned}
& |y|=2^{n+1}, \quad|b|=2^{r} \quad \text { where } n \geq r \geq 3 ; \\
& y^{2}=a, y^{a}=y^{-1}, \quad b^{a}=b, \quad a^{2}=1 ; \\
& y^{\phi}=y^{-1+2^{n-r+1} i}, \quad b^{\phi}=b a^{2^{n-r}} ;, \quad a^{\phi}=a y, \quad \phi^{2}=b a^{2^{n-r-1} i} .
\end{aligned}
$$

Here $i$ and $j$ are odd.

Proof. By (vii), $\phi^{2}=b^{m} a^{2^{n-r-1} i}$ for odd $i$. Replacing $b$ by an odd power of $b$, we may assume $m$ is a power of 2 . Then

$$
y^{\phi}=\left(\alpha a^{\phi}\right)^{\phi}=y^{-1}\left[\alpha, \phi^{2}\right]=y^{-1}\left[a, a^{2^{n-r-1} i}\right]=y^{-1} a^{2^{n-r} i}=y^{-1+2^{n-r+1} i} \text {. }
$$

Also, $\phi$ centralizes $\phi^{2}$; now $b^{\phi}=b^{s} a^{2^{n-r} t}$ for odd $s, t$ since $V_{r} / V_{r-1}$ is operator-isomorphic to $W$, and so

$$
\begin{aligned}
b^{m} a^{2^{n-r-1} i} & =\left(b^{s} a^{2^{n-r} t}\right)^{m} a^{2^{n-r-1} i\left(-1+2^{n-r+1} i\right)} \\
& =b^{s m} a^{2^{n-r} t m K_{a} a^{-2^{n-r-1}} i+2^{2(n-r)} i^{2}},
\end{aligned}
$$

for odd $K$; hence $m=s m$ and

$$
2^{n-r} i=2^{n-r} t m K+2^{2(n-r)} i^{2} .
$$

The latter equation implies $m$ is odd, so $m=1$ and $s=1$. This, together with (v), proves (viii).

We will use again the transfer argument based on Grün's theorem. $\Phi(T)=$ $\langle y, b\rangle$ has index 4 in $T$, while $T^{\prime}=\langle y\rangle$. Now 
$y^{b}=\left(a \phi^{-1} \alpha \phi\right)^{b}=\alpha\left(\phi^{-1}\right)^{b} a \phi^{b}=\alpha\left(\phi a^{-2^{n-r} j}\right)^{-1} \alpha\left(\phi a^{-2^{n-r} j}\right)=a a^{2^{n-r} j} \phi^{-1} a \phi a^{-2^{n-r} j}$.

Write $k=-1+2^{n-r+1} i$, so that $y^{\phi}=y^{k}$; then $y^{\phi-1}=y^{m}$ where $y^{k m}=y$. Then

$$
\begin{aligned}
y^{b} & =a \phi^{-1} a^{2^{n-r} j m} a \phi a^{-2^{n-r} j}=a \phi^{-1} a a^{-2^{n-r} j m} \phi a^{-2^{n-r} j} \\
& =a \phi^{-1} a \phi a^{-2^{n-r} j m k} a^{-2^{n-r} j}=y a^{-2^{n-r+1} j} .
\end{aligned}
$$

It follows that $\Phi(\Phi(T))=\left\langle y^{2}, b^{2}\right\rangle=\left\langle a, b^{2}\right\rangle$.

If $x \in a \phi \Phi(T)$, then $x^{2} \equiv(a \phi)^{2} \bmod \Phi(\Phi(T))$, since $a$ and $\phi$ both centralize $\Phi(T) / \Phi(\Phi(T))$. Now

$$
\begin{aligned}
& (a \phi)^{2}=a \phi^{-1} \phi^{2} a \phi=a \phi^{-1}\left(b a^{2^{n-r-1} i}\right) a \phi \equiv y b \bmod \Phi(\Phi(T)) ; \\
& (y b)^{2}=y b y b=y^{2} b[b, y] b=y^{2} b a^{2^{n-r+1}} j_{b} \equiv a \bmod \left(a^{2}, b^{2}\right) .
\end{aligned}
$$

Hence $|y b|=2^{n+1}$; also, every element of $y b \Phi(\Phi(T))$ has order $2^{n+1}$, and $|x|=$ $2^{n+2}>|y|$, so $x$ is not conjugate to any element of $\langle y\rangle$.

Suppose $x \in \phi \Phi(T)$. Then $x=\phi b^{s} y^{t}$ for some $s, t$ and

$$
\begin{aligned}
& x^{2}=\phi^{2}\left(b^{\phi}\right)^{s}\left(y^{\phi}\right)^{t} b^{s} y^{t}=\phi^{2}\left(b^{\phi}\right)^{s} y^{\left(-1+2^{n-r+1} i\right) t} b^{s} y^{t} \\
& =\phi^{2}\left(b^{\phi}\right)^{s} b^{s} y^{2^{n-r+1} u t}, \text { for some odd } u \text {; } \\
& =b a^{2^{n-r+1} i}\left(b a^{2^{n-r} j}\right)^{s} b^{s} a^{2^{n-r} u t} \equiv b a^{2^{n-r-1}} \bmod \left\langle b^{2}, a^{2^{n-r}}\right\rangle . \\
& \left(x^{2}\right)^{2^{r-1}}=\left(b a^{2^{n-r-1}} z\right)^{2^{r-1}} \text {, for some } z \in\left\langle b^{2}, a^{2^{n-r}}\right\rangle \text {; } \\
& =b^{2^{r-1}} a^{2^{n-2} k}, \quad \text { for some odd } k \text {. }
\end{aligned}
$$

So if $x$ is conjugate to an element of $\langle y\rangle$, then $a^{2^{n-2}} \sim a^{2^{n-2}} b^{2^{r-1}} \cdot \mathbf{C}_{T}\left(a^{2^{n-2}}\right)$ $=\langle a \phi, y, b\rangle$, and $\mathrm{C}_{T}\left(a^{2^{n-2}} b^{2^{r-1}}\right)=\langle\phi, y, b\rangle$. By Sylow's theorem, $\langle a \phi, y, b\rangle$ $\cong\langle\phi, y, b\rangle$; but the former has exponent $2^{n+2}$ while the latter has exponent $2^{n+1}$.

It follows that $T$ cannot be generated by conjugates of elements of $T^{\prime}$, and so Grün's theorem is violated.

This completes the proof of Theorem A.

We will now assume that the involutions of $W$ fall into three distinct $G$-conjugacy classes. Clearly the direct product $G$ of two simple groups whose Sylow 2-subgroups are dihedral or semidihedral of sufficiently large order will have such a configuration for $W$. We will show that this is the only possibility for the Sylow 2-subgroup of $G$ :

Theorem B. Let $T$ be a Sylow 2-subgroup of a finite fusion-simple group G. Assume that $T$ bas no normal elementary subgroup of order $8 ; N_{G}(T)=T C_{G}(T)$; 
$T \approx D_{8}$; and the involutions of the unique normal four-group $W$ of $T$ fall into three G-classes. Then $T$ is the direct product of two groups each of which is dibedral or semidibedral.

Proof of Theorem B. By Theorem 1, $T$ has a normal subgroup $V \cong \mathbf{Z}_{4} \times \mathbf{Z}_{4}$; and $\mathbf{C}_{T}(V)$ is a metacyclic group by Alperin [1].

We shall use the following supplement to Lemma 6:

Lemma 7. Let $L$ be a metacyclic 2-group containing a central subgroup $V \cong$ $\mathbf{Z}_{4} \times \mathbf{Z}_{4^{*}}$. Then

(a) For $j=1,2, \ldots$ let $V_{j}=\Omega_{j}(L)$. Suppose $\left[V_{i}: V_{i-1}\right]=4$ but $\left[V_{j}: V_{j-1}\right]$ $\leq 2$ for $j \geq i+1$ (as in Lemma $6(\mathrm{e})$ ). Then $L / V_{i-2}$ is Abelian.

(b) Let $\leqq$ be an automorphism of $L$ which inverts $a, b \in L$ where $L=\langle a, b\rangle$. Then $\supseteq$ inverts $V$.

Proof. (a) Let $\left[L: V_{i}\right]=2^{r}$; then $L$ can be generated by two elements $a, y$ where $y \in V_{i}$ has order $2^{i}$ and $a^{2^{r}}=x \in V_{i}$ has order $2^{i}$. Since $L / V_{i}$ is Abelian, $[a, y] \in V_{i}$, and we claim that $[a, y] \in V_{i-2}$. If not, then $[a, y]$ has order $2^{e}$ where $e>i-2$, and by Lemma 6(c),

$$
\left|\left[a, y^{2^{i-2}}\right]\right|=2^{-i+2}|[a, y]|=2^{e-i+2}>1,
$$

contradicting $\Omega_{2}(L) \leq \mathbf{Z}(L)$.

(b) One of $a$ and $b$, say $a$, must be of maximal order in $L$. Then there is $y \in L$ such that $\left\langle a^{2^{r}}, y\right\rangle=V_{i},\langle a, y\rangle=L$, and $\left\langle\Omega_{2}(\langle a\rangle), \Omega_{2}(\langle y\rangle)\right\rangle=V$. Clearly $\supseteqq$ inverts $\Omega_{2}((a))$. We claim $\supseteqq$ inverts $\Omega_{2}((y))$. For, replacing $y$ by an element of $y V_{i-1}$ if necessary, we may assume $b=a^{m} y$ for some number $m$. Then

$$
\begin{aligned}
y & =a^{-m} a^{m} y=a^{-m} b ; \\
y^{\mathcal{D}} & =\left(a^{-m} b\right)^{\mathcal{D}}=a^{m} b^{-1}=b^{-1} a^{m}\left[a^{m}, b^{-1}\right]=y^{-1} z,
\end{aligned}
$$

for some $z \in L^{\prime}$. By (a), $L^{\prime} \leq V_{i-2}$, so $|z| \leq 2^{i-2}$. Now

$$
\left(y^{-1} z\right)^{2}=y^{-2} z\left[z, y^{-1}\right] z=y^{-2} z^{2 e},
$$

where $e$ is odd, since $\left[z, y^{-1}\right] \equiv 1 \bmod \left\langle z^{4}\right\rangle$. By induction, $\left(y^{-1} z\right)^{2^{k}}=y^{-2^{k}} z^{2^{k} f}$, for odd $f$. Taking $k=i-2$, we have

$$
\left(y^{2^{i-2}}\right)^{\mathfrak{C}}=\left(y^{-1} z\right)^{2^{i-2}}=y^{-2^{i-2}} z^{2^{i-2} f} .
$$

But $z^{2^{i-2}}=1$, so $\supseteq$ inverts $\Omega_{2}(\langle y\rangle)$ as claimed.

As in the proof of Theorem A, we will use fusion patterns in $E_{16}$ 's of $T$. 
The first task is to establish that $T$ has $E_{16}$ 's of a suitable kind. Define

$Z=\{x \in G: x$ is conjugate to a nonidentity element of $W\}$.

If $\gamma \in \mathfrak{Z} \cap T-W$, define

$$
W(\gamma)=\left\{x \in W: \gamma \sim_{G} \gamma x\right\} .
$$

(i) $T$ has a subgroup $F \cong E_{16}$ generated by $G$-conjugates of elements of $W$.

Proof. By Glauberman's $Z^{*}$-theorem, $T-W$ contains $G$-conjugates of each element of $W^{\#}$.

Assume (i) is false. Let $\omega^{*} \in T-W, \omega^{*} \sim \omega \in W$. Let $T^{*}$ be a Sylow 2subgroup of $\mathbf{C}_{G}\left(\omega^{*}\right)$ containing $\left\langle W, \omega^{*}\right\rangle$. If $W^{*} \cap W=1$ then $F=W^{*}$ will do; so $W^{*} \cap W \neq 1$. $W^{*} \cap W \neq\langle\omega\rangle$, since $W^{*}$ only contains one $G$-conjugate of $\omega$. Let $W^{*} \cap W=\langle\eta\rangle$; then the seven involutions of $W^{*}$ are $\omega, \xi, \eta, \omega^{*}, \xi^{*}, \eta^{*}=\eta$. $\omega^{*} \omega$, and $\omega^{*} \xi$. Now $\omega^{*} \sim_{V} \omega^{*} z$ for some $z \in W^{\#}$, and $z \neq \eta$ since $\omega^{*} \not \omega^{*} \eta^{*}$. So there is $v \in V$ such that either $\left(\omega^{*}\right)^{\nu}=\omega^{*} \omega$, and hence $\left(\xi^{*}\right)^{\nu}=\xi^{*} \omega$, or $\left(\omega^{*}\right)^{\nu}$ $=\omega^{*} \xi$, and hence $\left(\xi^{*}\right)^{\nu}=\xi^{*} \xi_{\text {. }}$.

We have proved: If (i) is false, then for any $\gamma \in T \cap B-W$, we have $|W(\gamma)|$ = 2; and moreover, if $z \in W, W(y z)=W(y)$.

Suppose there are $\gamma, \delta \in T \cap \not{-} W$ with $W(\gamma) \neq W(\delta)$. Then $\gamma$ and $\delta$ commute by Lemma 5 , and the above remark implies that $\langle W, \gamma, \delta\rangle$ is not of order 8 . Hence $F=\langle W, \gamma, \delta\rangle$ satisfies (i).

So we may assume that

(i.i) There is $\omega \in W$ with $W(\gamma)=\langle\omega\rangle$ for all $\gamma \in T \cap \not-W$ :

We now prove

(i.ii) If $\gamma \in \operatorname{ccl}_{G}(\omega) \cap(T-W)$, then $\omega$ is not a square in $\mathbf{C}_{T}(\gamma)$.

Proof. Let $K=\langle\omega, \gamma\rangle$. Then there is $v \in V$ with $\gamma^{v}=\gamma \omega$ and $\omega^{v}=\omega$. But if $T^{*}$ is a Sylow 2-subgroup of $\mathbf{C}_{G}(\gamma)$ containing $K$, then $K \cap W^{*}=\langle\gamma\rangle$, so there is $u \in V^{*}$ with $\omega^{u}=\omega \gamma$ and $\gamma^{u}=\gamma$. Hence $A_{G}(K) \cong \Sigma_{3}$.

Let $N=\mathrm{N}_{G}(K), C=\mathrm{C}_{G}(K)$, and let $S$ be a Sylow 2-subgroup of $C$ which contains $\mathbf{C}_{T}(\gamma)$. Then $N=C N_{N}(S)$ and $N / C \cong \Sigma_{3}$, so $N_{N}(S) / N_{N}(S) \cap C \cong \Sigma_{3^{\prime}}$. Hence $N$ contains a 3-element acting on $S$ so as to cycle $K$. But $\Phi(S) \leq$ the Frattini subgroup of some Sylow 2-subgroup of $T$, so is metacyclic, and does not admit an automorphism of order 3 from $G$. So $K \cap \Phi(S)=1$ and $\omega$ is not a square in $S$, which proves (i.ii).

(i.iii) If $\gamma \in Z \cap(T-W)$, then $\gamma W$ contains some conjugate of $\omega$.

Proof. Let $E=\langle W, \gamma\rangle$, and let $T^{*}$ be a Sylow 2-subgroup of $\mathbf{C}_{G}(\gamma)$ containing $E$. Then $W^{*} \cap E \geq\langle y\rangle$. If $W^{*} \cap E=\langle y\rangle$ then $W^{*} \cap W=1$ and so $\left\langle W^{*}, W\right\rangle=F$ satisfies (i). Hence $W^{*} \leq E$ and $\left|W^{*} \cap W\right|=2$. We need only show $\omega^{*} \notin W$, i.e., 
$W^{*} \cap W \neq\langle\omega\rangle$. But $\gamma \sim \gamma \omega$ in $T$, so $\omega \in W^{*}$ would give two conjugate elements of $W^{*}$.

Let $\tilde{G}$ be the set of all subgroups $E$ of $T$ where $E \cong E_{8}$ and $E=\langle E \cap \supsetneqq\rangle$. Every $E \in \mathbb{G}$ contains W. Moreover

(i.iv) If $E \in \mathcal{G}$, then $A_{G}(E) \cong \Sigma_{3}$, and the three classes of involutions of $E$ are $\{\eta\}, E_{1}^{\#}$, and $\eta E_{1}^{\#}$, where $\eta \in W-\langle\omega\rangle$ and $E_{1}^{\#}$ consists of conjugates of $\omega$. ( $E_{1}$ is a four-subgroup of $\left.E_{\text {. }}\right)$

Proof. By (i.iii), $E=\langle W, \gamma\rangle$ where $\gamma \sim \omega$. Let $T^{*}$ be a Sylow 2-subgroup of $\mathrm{C}_{G}(\gamma)$ containing $E$. Then $W^{*} \leq E$, and so there are $v, u \in V, V^{*}$ respectively with $\gamma^{\nu}=\gamma \omega$ and $\omega^{u}=\omega \gamma ; v$ and $u$ both centralize $W^{*} \cap W=\langle\eta\rangle$ say. Hence $v$ and $u$ generate $\Sigma_{3}$ as claimed in (i.iv).

We now introduce $H=\langle\supset \cap T\rangle \triangleleft T$ :

(i.v) Let $H=\langle\nexists \cap T\rangle$ and let $2^{a}=\max \{|\gamma \delta|: \gamma, \delta \in \nexists \cap T\}$. Then $a \geq 2$, and $H$ is the direct product of $\langle\pi\rangle$ and a dihedral group of order $2^{a+1}$, for some $\pi \in W-\langle\omega\rangle$. (It then follows from (i.iii) that $H=\langle\pi\rangle \times\langle\gamma, \delta\rangle$ for some $\gamma, \delta \epsilon$ $\operatorname{ccl}_{G}(\omega) \cap(T-W)$.)

Proof. If $a=1$, then $H$ is elementary Abelian and $>W$, so $T$ has a normal $E_{8}$.

If $\gamma, \delta \in \bigcap \cap T$, let $\sigma=\gamma \delta$; if $|\sigma| \geq 4$ then $\Omega_{1}(\langle\sigma\rangle) \leq \Phi(T)$, so $\Omega_{1}(\langle\sigma\rangle) \leq$ $W$ and is equal to $\langle\omega\rangle$.

By (i.iii), there are $\gamma, \delta \in \operatorname{ccl}_{G}(\omega) \cap(T-W)$ such that $|\gamma \delta|=2^{a}$.

Suppose $a=2$. Then $H$ is non-Abelian, so $|H| \geq 2^{4}$. But also $H /\langle\omega\rangle$ is elementary, so $|H| \leq 2^{5}$ by the four-generator theorem of [12]. Let $\gamma, \delta \epsilon$ $\operatorname{ccl}_{G}(\omega) \cap(T-W)$ with $|\gamma \delta|=4$, so $D=\langle\gamma, \delta\rangle \cong D_{8}$ and $H=D \circ \mathrm{C}_{H}(D)$. But $\mathbf{C}_{H}(D)$ is elementary by (i.ii). Hence $\mathbf{C}_{H}(D)=\mathbf{Z}(H) \triangleleft T$, so $\mathbf{C}_{H}(D)=W$ and $H=D \times\langle\pi\rangle$ for some $\pi \in W-\langle\omega\rangle$.

Suppose $a \geq 3$, and let $\gamma, \delta \in ß \cap(T-W)$ with $\sigma=\gamma \delta$ of order $2^{a}$. Then $\Omega_{2}(\langle\sigma\rangle)$ is a subgroup of order 4 in $V$. Let $B=\left\langle\Omega_{2}(\langle\sigma\rangle), W\right\rangle ; B \leq V \cap H$, and $B \triangleleft T$ since all subgroups of order 8 in $V$ are normal in $T$.

Let $B \leq A \leq H$ where $A \triangleleft T$ and $A$ is a maximal Abelian subgroup of $H$. Suppose that $H$ contains no normal $\mathrm{Z}_{4} \times \mathrm{Z}_{4}$ of $T$. Then $A$ is of type $\left(2^{r}, 2\right)$ for some $r$. Also $A=\mathrm{C}_{H}(B)$; for if not, then $r \geq 3$, and taking $A<R \leq \mathrm{C}_{H}(B)$ with $[R: A]=2$ and $R \triangleleft T$, we get that $\Omega_{2}(R)$ is Abelian of type $(4,2,2)$ or $(4,4)$, both of which are impossible. Now (i.i) implies that all $\gamma \in Z \cap(T-W$.) induce the same automorphism of $B$. Hence, all such $\gamma$ are congruent $\bmod A=$ $\mathrm{C}_{H}(B)$. It follows that $[H: A]=2$ and $H=\langle\pi\rangle \times\langle\gamma, \delta\rangle$ where $\pi \in W-\langle\omega\rangle$ and $\gamma, \delta \in Z \cap(T-W)$ with $|\gamma \delta|$ maximal, proving (i.v). (This is the same argument as in the proofs of (iii) and (iv) in Theorem A, Case 1.)

Hence it will suffice to show that $H$ contains no normal $\mathrm{Z}_{4} \times \mathrm{Z}_{4}$ of $T$, and 
we will do this now. Suppose $H \geq R \cong \mathrm{Z}_{4} \times \mathrm{Z}_{4}$ where $R \triangleleft T$. Then $\Omega_{1}(R)=W$. Now by (i.iii), $H=\left\langle W, \operatorname{ccl}_{G}(\omega) \cap(T-W)\right\rangle$, so $R \leq H$ gives $W \leq \Phi(H)$ and therefore $H=\left\langle\operatorname{ccl}_{G}(\omega) \cap(T-W)\right\rangle$. Let

$$
\mathscr{U}=\operatorname{ccl}_{G}(\omega) \cap(T-W), \quad H_{0}=H \cap \mathrm{C}_{T}(R),
$$

so that $R \leq H_{0}$.

For any $\gamma \in \mathcal{U},[R, \gamma]=\langle\omega\rangle$, and it follows that there are exactly three automorphisms which $\gamma \in \mathfrak{U}$ can induce on $R$, and they are distinguished only by $\mathbf{C}_{R}(\gamma)$, which has order 8. Hence, $2 \leq\left[H: H_{0}\right] \leq 4$.

(a) It cannot happen that there is $\gamma \in \mathfrak{U}$ such that $H_{0}$ is generated by two elements both of which $\gamma$ inverts.

Proof. If so, then $\gamma$ inverts $R$, by Lemma $7(b)$. But then $w(\gamma)=w$, contradicting (i.i).

(b) $\left[H: H_{0}\right]=4$.

Proof. Suppose $\left[H: H_{0}\right]=2$. Then all $\gamma \in \mathfrak{U}$ are congruent $\bmod H_{0}$, so $H$ $=\left\langle x y: x y \in \mathfrak{N}, x \in H_{0}\right\rangle=\left\langle\left\{x: x \in H_{0}, x y \in \mathfrak{W}\right\}, y\right\rangle$, where $\gamma$ is some fixed member of $\mathfrak{X}$. $\left\{x: x \in H_{0}, x y \in \mathfrak{U}\right\}$ is $\gamma$-invariant, and so the group it generates has index 2 in $H$, so $H_{0}=\left\langle x: x \in H_{0}, x y \in \mathfrak{U}\right\rangle$. As $H_{0} / \Phi\left(H_{0}\right)$ is a four-group, $H_{0}=\langle x, y\rangle$ where $x y, y \gamma \in \mathfrak{U}$. But then $y$ inverts both $x$ and $y$, contradicting (a).

By (i.ii), the automorphism $\supseteqq$ of $R$ with $[R, \supseteqq]=\langle\omega\rangle$ and $\mho_{1}\left(C_{R}(\supseteq)\right)=\langle\omega\rangle$ cannot be induced by an element of $\mathscr{U}$. Hence there are $\gamma$ and $\delta \epsilon \mathscr{U}$ inducing distinct automorphisms of $R$, and $H_{0}(\gamma \delta)$ contains no elements of $\mathscr{U}$. Then

$$
H=\left\langle H_{0}, \gamma, \delta\right\rangle, \quad \mathscr{U}=\left(H_{0} \gamma \cap \mathfrak{U}\right) \cup\left(H_{0} \delta \cap \mathscr{U}\right) .
$$

(c) There are $x, y \in H_{0}$ with $x y \in \mathfrak{X}, y \delta \in \mathfrak{U}$, and $H_{0}=\langle x, y\rangle$.

Proof.

$$
\begin{aligned}
H & =\left\langle H_{0} \gamma \cap \mathfrak{U}, H_{0} \delta \cap \mathfrak{U}\right\rangle=\left\langle x y, y \delta: x, y \in H_{0}, x y \in \mathfrak{U}, y \delta \in \mathfrak{U}\right\rangle \\
& =\left\langle\left\{x, y: x, y \in H_{0}, x y \in \mathfrak{U}, y \delta \in \mathfrak{U}\right\}, y, \delta\right\rangle .
\end{aligned}
$$

By (a), $\left\langle x: x \in H_{0}, x y \in \mathfrak{U}\right\rangle \leq$ some maximal subgroup $X$ of $H_{0}$, and $\left\langle y: y \in H_{0}\right.$,

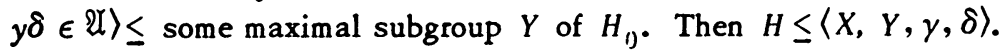

Suppose $\gamma$ and $\delta$ centralize $H_{0} / \Phi\left(H_{0}\right)$; then they normalize $X$ and $Y$, so $[H:\langle X, Y\rangle]=4$, so $\langle X, Y\rangle=H_{0}$, and (c) follows. Hence, we may assume $\gamma$ or $\delta$ fails to centralize $H_{0} / \Phi\left(H_{0}\right)$.

Suppose $\gamma$, say, does not centralize $H_{0} / \Phi\left(H_{0}\right)$. By Lemma $7(a), H_{0}$ has a quotient $\hat{H}_{0} \cong \mathbf{Z}_{2 r} \times \mathbf{Z}_{4}$, for some $r \geq 2$. If $r=2$, then $H_{0} / \Phi\left(H_{0}\right)$ is operatorisomorphic to $W$ (Lemma $G(\mathrm{f}))$, so $\gamma$ must centralize $H_{0} / \Phi\left(H_{0}\right)$. Hence $\hat{H}_{0}=$ $\langle\hat{a}\rangle \times\langle\hat{b}\rangle$ with $|\hat{a}|=2^{r} \geq 8,|\hat{b}|=4$, and 


$$
\hat{a}^{\gamma}=\hat{a}^{i} \hat{b}^{j}, \quad \hat{b}^{\gamma}=\hat{a}^{2^{r-2} k} \hat{b}^{l},
$$

where $i, j$, and $l$ are all odd. We claim no $\hat{a}^{m} \hat{b}^{n}$ with $m$ odd is inverted by $\gamma$, which implies that $H_{0} \gamma \cap \mathfrak{U} \leq\left\langle\Phi\left(H_{0}\right), b\right\rangle \gamma$ (where $b \Phi\left(H_{0}\right)=\hat{b}$ ). Namely, taking $m=1$,

$$
\left(\hat{a} \hat{b}^{n}\right)^{\gamma}=\left(\hat{a}^{i} \hat{b}^{j}\right)\left(\hat{a}^{2^{r-2} k} \hat{b}^{l}\right)^{n}=\hat{a}^{i+2^{r-2} k n} \hat{b}^{j+l n} .
$$

So if $\gamma$ inverts $\hat{a} \hat{b}^{n}$, then

$$
-n \equiv j+l n \quad \bmod 4, \quad-n(l+1) \equiv j \bmod 4 ;
$$

but $l+1$ is even and $j$ is odd.

If neither $\gamma$ nor $\delta$ centralizes $H_{0} / \Phi\left(H_{0}\right)$, then

$$
H=\left\langle H_{0} \gamma \cap \mathscr{Q}, H_{0} \delta \cap \mathscr{U}\right\rangle \leq\langle B \gamma, B \delta\rangle \leq\langle B, \gamma, \delta\rangle<H,
$$

where $B=\left\langle\Phi\left(H_{0}\right), b\right\rangle$ (so $\gamma$ and $\delta$ normalizes $B$ ).

So we may assume $\gamma$ centralizes $H_{0} / \Phi\left(H_{0}\right)$ and $\delta$ does not. Then

$$
H \leq\left\langle H_{0} \gamma \cap \mathbb{R}, B \delta \cap \mathcal{U}\right\rangle \leq\left\langle\left\{x: x y \in \mathcal{Q}, x \in H_{0}\right\}, B, y, \delta\right\rangle \text {. }
$$

Now $\left\{x: x y \in \mathfrak{U}, x \in H_{0}\right\} \leq$ some maximal subgroup $X$ of $H_{0}$, so $H \leq\langle X, B, \gamma, \delta\rangle$. $X \neq B$, since then $H \leq\langle B, \gamma, \delta\rangle$. Hence there is $x \in X$ with $x \notin \Phi\left(H_{0}\right), x y \in \mathcal{U}$, and $x^{\delta} \equiv x b \bmod \Phi\left(H_{0}\right)$. Then $(x y)^{\delta}=x b z \gamma^{\delta}$, for some $z \in \Phi\left(H_{0}\right)$. But $\gamma^{\delta} \in H_{0} \gamma$ $\cap \mathfrak{U} \leq X y$, so $\gamma^{\delta}=u y$ for some $u \in X$; then $(x y)^{\delta}=x b z u y=t \gamma$, where $t=x b z u \equiv$ $x b$ or $b \bmod \Phi\left(H_{0}\right)$. But then $H_{0}=\langle x, t\rangle$, contrary to (a).

(d) Take $x, y \in H_{0}$ with $x y \in \mathfrak{U}, y \delta \in \mathfrak{U}$, and $H_{0}=\langle x, y\rangle$. Then the set of all elements of $H_{0}$ inverted by $\gamma$ is $\langle x, W\rangle$, and ditto for $\delta$ is $\langle y, W\rangle$. $\Omega_{2}(\langle x, W\rangle)$ $=\Omega_{2}(\langle y, W\rangle)=$ the unique maximal subgroup of $R$ inverted by $\gamma$ and $\delta$ (namely, the one in which $\omega$ is a square).

Proof. Suppose $u \in H_{0}$ has $u \notin\langle x, W\rangle, u^{2} \epsilon\langle x, W\rangle$, and $u^{\gamma}=u^{-1}$. No element of $x\left\langle x^{2}, W\right\rangle$ is a square in $H_{0}$, as $x$ is a generator for $H_{0}$, so $u^{2} \epsilon\left\langle x^{2}, W\right\rangle$. If $\langle x, W\rangle=\langle x, z\rangle$ for $z \in W-\langle x\rangle$, then $u^{2}=z^{i} x^{2 j}$ for some $i$, $j$, so by Lemma $6(\mathrm{~g})$, $u \equiv v^{i} x^{j} \bmod W$, where $v^{2}=z$. As $u \notin\langle W, x\rangle, u \equiv v x^{j} \bmod W$. But then $\langle u, x\rangle$ is an Abelian group which contains $R$, so $\gamma$ inverts $R$, and $W(\gamma)=W$, contrary to (i.i).

(e) $(y \delta)^{2} \in \Phi\left(H_{0}\right)$.

Proof. Certainly $(\gamma \delta)^{2} \in H_{0}$. If $(\gamma \delta)^{2} \notin \Phi\left(H_{n}\right)$, then $\gamma$ and $\delta$ would both invert the same subgroup of $H_{0}$, namely $\left\langle(y \delta)^{2}, W\right\rangle$, so $x$ and $y$ would not generate $H_{0}$.

(f) $\langle x, W\rangle=\left\{u \in H_{0}: u\right.$ is inverted by every element of $\left.H_{0} \gamma \cap \mathfrak{U}\right\}$. Hence $\delta$ normalizes $\langle x, W\rangle$. 
Proof. Let $t y \in H_{0} \gamma \cap \mathscr{U}$; then $t \in\langle x, W\rangle$, so $t$ centralizes $\langle x, W\rangle$ and $t y$ inverts $\langle x, W\rangle$. Applying (d) to ty instead of $\gamma$, we get (f).

(g) $H_{0}=R$.

Proof. Suppose $H_{0}>R$. One of $x$ and $y$ has maximal order in $H_{0}$; suppose it is $x$, so $|x| \geq 8$. By (f), $x^{\delta}=x^{i} z$, where $i= \pm 1$ and $z \in W ;\left(x^{2}\right)^{\delta}=x^{ \pm 2}$. Since $\delta$ inverts $\Omega_{2}(\langle x\rangle)$, the minus sign must occur, and $x^{\delta}=x^{-1} z$, for some $z$ $\epsilon W$. Moreover, $\delta$ inverts $\left\langle x^{2}, W\right\rangle$ so $\left\langle x^{2}, W\right\rangle \leq\langle y, W\rangle \cap \Phi\left(H_{0}\right)=\left\langle y^{2}, W\right\rangle$. $|x| \geq$ $|y|$, so $\left\langle x^{2}, W\right\rangle=\left\langle y^{2}, W\right\rangle$, and $H_{0}=\langle x, y, W\rangle$ contains $\left\langle x^{2}, W\right\rangle$ with index 2 .

Since $R \leq H_{0}, H_{0}$ is Abelian of type $\left(2^{r}, 4\right)$, with $|x|=|y|=2^{r}$ and $r \geq 3$. Applying the same arguments to $y$, we get $x^{\delta}=x^{-1} z$ and $y^{\gamma}=y^{-1} w$, for $z, w \in W$.

By $(\mathrm{e}),(\gamma \delta)^{2} \in \Phi\left(H_{0}\right)$. We will show that there are $t, u \in H_{0}$ with $t \gamma, u \delta \in \mathscr{V}$ and $(t \gamma)(u \delta)$ an involution, which means $\langle W, t \gamma, u \delta\rangle=F$ satisfies (i).

Namely, $\gamma x^{i}$ and $\delta y^{j} \in \mathfrak{U}$ for all $i, j$; and

$$
\begin{aligned}
\gamma x^{i} \delta y^{j} & =\gamma \delta\left(x^{-1} z\right)^{i} y^{j}=\gamma \delta x^{-i} z^{i} y^{j} \\
\left(\gamma x^{i} \delta y^{j}\right)^{2} & =(y \delta)^{2}\left(x^{-i} z^{i} y^{j}\right)^{\gamma \delta} x^{-i} z^{i} y^{j}=(\gamma \delta)^{2} x^{-2 i} y^{2 j} z^{i} w^{j} .
\end{aligned}
$$

Now there is $v \in R$ such that $H_{0}=\langle v\rangle \times\langle y\rangle$ and $\gamma$ centralizes $v$ while $\delta$ centralizes $v y^{2^{r-2}}$. We may choose $v$ and the generators $x$ and $y$ so that $x y^{-1}=$ $v$. Then

$$
\left(y x^{i} \delta y^{j}\right)^{2}=(\gamma \delta)^{2} x^{-2 i} y^{2 i-2 i+2 j} z^{i} w^{j}=(y \delta)^{2} v^{-2 i} y^{2(j-i)} z^{i} w^{j}
$$

But also

$$
\begin{gathered}
v=v^{\gamma}=\left(x y^{-1}\right)^{\gamma}=x^{-1} y w=v^{-1} w ; \\
v y^{2^{r-2}}=\left(v y^{2^{r-2}}\right)^{\delta}=\left(x y^{-1} y^{2^{r-2}}\right)^{\delta}=x^{-1} z y y^{-2^{r-2}}=\left(v y^{2^{r-2}}\right)^{-1} z ;
\end{gathered}
$$

so $w$ and $z$ are the squares in the subgroups of $R$ centralized by $\gamma$ and $\delta$, i.e., $w=v^{2}$ and $z=v^{2} \omega$. Hence,

$$
\left(\gamma x^{i} \delta y^{j}\right)^{2}=(y \delta)^{2} v^{-2 i} y^{2(j-i)}\left(v^{2} \omega\right)^{i} v^{2 j}=(\gamma \delta)^{2} y^{2(j-i)} v^{2 j} \omega^{i}
$$

where $\langle\omega\rangle=\Omega_{1}(\langle y\rangle)$. Whatever the value of $(y \delta)^{2} \in \Phi\left(H_{0}\right), i$ and $j$ can be chosen so that $\left(y x^{i} \delta y^{j}\right)^{2}=1$, as claimed.

(h) Final step in the proof of (i.v).

We have $H_{0}=R=\langle x, y\rangle$; but by (d), $\langle x, W\rangle=\langle y, W\rangle$, so $\left.R\right\rangle\langle x, y\rangle$.

Let $H=\langle\pi\rangle \times\langle\gamma, \delta\rangle$ as in (i.v). Then $H$ has two classes of $E_{8}$ 's, represented by $E=\langle W, \gamma\rangle$ and $F=\langle W, \delta\rangle$. By (i.iv), $E$ and $F$ each contain a unique involution-say $\alpha, \beta$ respectively-of $W-\langle\omega\rangle$, which is the only representative of its 
conjugacy class in $E, F$ respectively. If $\alpha=\beta$ then $\alpha$ is the only representative of its class in $T$, contrary to the $\mathbf{Z}^{*}$-theorem. Hence $\alpha$ and $\beta$ are $\pi$ and $\pi \omega$, and $E$ and $F$ are not $G$-conjugate.

(i.vi) $\mathbf{C}_{T}(H)=W$.

Proof. As $\mathbf{C}_{T}(H) \triangleleft T$, it suffices to show that $\mathbf{C}_{T}(H)$ is elementary. Let $\beta$ be an element of order 4 in $\mathrm{C}_{T}(H)$. $\beta^{2} \in \Phi(T) \leq \mathrm{C}_{T}(V)$, and as $\beta^{2}$ is an involution, we have $\beta^{2} \in W$. Then $\beta^{2} \in W-\langle\omega\rangle$, by (i.ii). Suppose $\pi=\beta^{2}$ and $\pi$ is the isolated involution of $E$. Let $S$ be a Sylow 2-subgroup of $\mathrm{C}_{G}(F)$ which contains $\langle F, \beta\rangle$; let $N=\mathbf{N}_{G}(F)$, so $N=\mathbf{N}_{N}(S) C_{G}(F)$. Now $\beta^{2}=\pi=(\pi \omega) \omega$. By (i.iv), $N$ is transitive on $\{(\pi \omega) \omega,(\pi \omega) \delta,(\pi \omega) \omega \delta\}$. Hence $\mathbf{N}_{N}(S)$ is transitive on this set, so $\beta^{2} \epsilon \Phi(S)$ implies $\{(\pi \omega) \omega,(\pi \omega) \delta,(\pi \omega) \omega \delta\} \leq \Phi(S)$, which is impossible as $\Phi(S)$ contains no $E_{8}$ 's.

Since $E$ and $F$ are $G$-conjugate, $T=H C_{T}(E)$. Now $H=\langle\pi\rangle \times\langle\gamma, \delta\rangle$ and $\gamma$ $\not \gamma \pi, \gamma \not \gamma \pi \omega, \delta \not \delta \pi, \delta \not \delta \pi \omega$ (by (i.i)). Hence $\langle\gamma, \delta\rangle=\left\langle\operatorname{ccl}_{G}(\omega) \cap T\right\rangle \triangleleft T$, and so $\langle\gamma \delta\rangle \triangleleft T$.

Let $C_{1}=\left\{x \in \mathbf{C}_{T}(E):(y \delta)^{x} \equiv(y \delta) \bmod \left\langle(\gamma \delta)^{4}\right\rangle\right\}$. Since $\langle y \delta\rangle \triangleleft T$ and $\gamma \in E$ inverts $\gamma \delta$, we have $T=H C_{1} . C_{1} / W$ is cyclic by (i.vi) and so $C_{1}$ is Abelian. But also $H=\langle\pi\rangle \times\langle\gamma, \delta\rangle$, so

$$
T=\langle\gamma, \delta\rangle C_{1} ; T /\langle\gamma, \delta\rangle \text { is Abelian. }
$$

Hence $\pi$ is a central involution of $T$ which is not in $T^{\prime}$. Hence $G$ has a subgroup of index 2, by Lemma 5.43 of [13].

This contradiction completes the proof of (i), so we have: $T$ contains subgroups $F \cong E_{16}$ with $F=\langle F \cap \supsetneqq\rangle$.

We now establish the fusion pattem in such an $E_{16}$ :

(ii) If $F \cong E_{16}$ and $F=\langle F \cap \gg\rangle$, then $A_{G}(F) \cong \Sigma_{3} \times \Sigma_{3}$.

Proof. Let $F \leq T, F \cong E_{16}$, and $F=\langle F \cap Z 3\rangle$. Lemma 3(ii) implies that $\mathbf{A}_{G}(F)$ is not a 2-group. We shall show that $A_{G}(F)$ is a $\{2,3\}$-group.

First suppose 7 divides $\left|A_{G}(F)\right|$. $W \leq F$, so $F^{\#}$ contains three distinct $G$ classes; so every element of $F^{\#}$ lies in $\$$, and all the 7-elements $\sigma$ of $A_{G}(F)$ have the same subgroups $A, B$ of $F$ as $[F, \sigma]$ and $\mathrm{C}_{F}(\sigma)$ respectively. The subgroup $K$ of $A_{G}(F)$ generated by its 7-elements lies in a copy of $L_{3^{\prime}}(2)$, acting completely reducibly on $F=A \times B$. $K$ is normal in $A_{G}(F)$, so $A_{G}(F)$ acts on $A$ $=[F, K]$ and $B=\mathrm{C}_{F}(K)$. Hence $\mathrm{A}_{G}(F)$ lies in a copy of $L_{3}(2)$ which acts completely reducibly. Let $T^{*}$ be a Sylow 2-subgroup of $G$ containing a Sylow 2-subgroup $U$ of $\mathbf{N}_{G}(F)$. Then $[U, F] \leq \Phi\left(T^{*}\right) \cap F=W^{*}$, and $\mathbf{C}_{F}(U) \leq \mathbf{C}_{F}\left(V^{*}\right)=W^{*}$. So $\langle B,[A, U]\rangle \leq W^{*}$, and as $B \cap[A, U]=1,[A, U]$ has order 2. The Sylow 2-subgroups of $L_{3}(2)$ do not have a commutator of order 2 on $A$. So $\left|A_{G}(F)\right|_{2}=2$ or 4 , 
and $\left|\mathbf{A}_{G}(F)\right|=7 \cdot 3 \cdot 2^{a}$ or $7 \cdot 2^{a}$, where $a=1$ or 2 . But $L_{3}(2)$ has no subgroups of these orders. Hence, 7 does not divide $\left|\mathbf{A}_{G}(F)\right|$.

We next show that $V$ induces a four-group on $F$. If not, then $\mathrm{C}_{V}(F)$ has order 8, and since $\mathrm{C}_{F}(V)=W$, there is $v \in V-\mathrm{C}_{V}(F)$, and a transversal $\{1, x$, $y, z\}$ to $W$ in $F$, such that $x^{\nu}=x \omega, y^{v}=y \eta$, and $z^{v}=z \xi$, where $W=\{\omega, \eta, \xi\}$. Take $\xi$ to be the involution of $\Phi\left(C_{V}(F)\right.$ ). By Lemma 3(ii) and (iii), $\xi$ is the only G-conjugate of $\xi$ to lie in $F$, and $\xi$ is central in $\mathbf{N}_{G}(F)$.

Now no $\pi \in z W$ lies in $\$; for if so, let $U$ be a Sylow 2-subgroup of $\mathbf{C}_{G}(\pi)$ which contains $F$; then $W(U) \leq F$ and $\mathbf{C}_{F}(V(U))=W(U)$ by Lemma 3(i). Hence $\xi \in W(U)$, so $W(U)=\langle\xi, \pi\rangle$, contrary to $\pi \sim \pi \xi$.

Since $F=\langle F \cap \bigcap\rangle$, we have $F=\langle w, \gamma, \delta\rangle$ where $\gamma, \delta \in Z,[\gamma, v]=\langle\omega\rangle,[\delta$, $V]=\langle\eta\rangle$, and $\gamma \delta W \cap \mathbb{Z}$ is empty.

Let $U$ be a Sylow 2-subgroup of $\mathbf{C}_{G}(\gamma)$ which contains $F$. As above, $W(U)$ $=\langle\xi, \gamma\rangle$. It follows that $\gamma \sim \omega$ or $\eta$ (not $\xi$ ), and $\gamma \not \gamma \xi \sim \eta$ or $\omega$. Now $\gamma \sim \gamma \omega$ and $\gamma \xi \sim \gamma \xi \omega=\gamma \eta$, so $\gamma W \leq \nsupseteq$. Similarly $\delta W \leq \mathfrak{Z}$. It follows that $\gamma \delta W=F^{\#}-F$ $\cap \mathfrak{\text { . }}$

Now since $\xi$ is central in $\mathbf{N}_{G}(F), A_{G}(F)$ has no 5-elements, and no fixed-point-free 3-elements, so is a $\{2,3\}$-group whose 3 -elements $\supseteqq$ have the following orbits on $F^{\#}: Y^{\#},\{\alpha\},\{\beta\},\{\alpha \beta\}, Y^{\#} \alpha, Y^{\#} \beta$, and $Y^{\#} \alpha \beta$, where $Y$ and $\langle\alpha, \beta\rangle$ are disjoint four-groups of $F . \gamma \delta W$ must be a union of some of these $\supseteqq$-orbits, since $\gamma \delta W=$ $F^{\#}-F \cap \precsim$. The product of the elements of $\gamma \delta W$ is 1 , and the only union of $\Omega$. orbits with this property and size 4 is (say) $Y^{\#} \alpha \cup\{\alpha\}=Y \alpha$. But then $W=\{x y$ : $x$ and $y \in \gamma \delta W\}=Y$; this is impossible as the elements of $W^{\#}$ are not G-conjugate to each other.

Now suppose 5 divides $\left|A_{G}(F)\right|$. The 5-elements of $L_{4}(2)$ partition $F^{\#}$ into three orbits of size 5, while the 3-elements partition it into five orbits of size 3 , or four of size 3 and three of size 1 . So if 3 divides $\left|A_{G}(F)\right|$ then $F^{\#}$ cannot contain three distinct classes, and $A_{G}(F)$ is a $\{2,5\}$-group. The only such subgroup of $L_{4}(2)$ with 2-part $\geq 4$ is a Frobenius group of order 20, but this contains no four-group, and $V$ induces a four-group on $F$.

Therefore, $A_{G}(F)$ is a $\{2,3\}$-group. We next show that $\mathbf{A}_{G}(F)$ acts fixedpoint-freely on $F^{\#}$. Suppose not; as $W=C_{F}(V)$, the fixed points of $A_{G}(F)$ all lie in $W$, and each is the only representative in $F$ of its $G$-conjugacy class (Lemma 3(ii)). If all three elements of $W^{\#}$ were fixed, then $\bigcap \cap F=W^{\#}$, so $\left.F\right\rangle\langle\not \cap \cap$ $F)$. It follows that precisely one element of $W$, say $\omega$, is fixed by $A_{G}(F)$.

Let $N=\mathbf{N}_{G}(F)$ and let $R / \mathbf{C}_{G}(F)=\mathbf{0}_{2}\left(\mathrm{~N} / \mathbf{C}_{G}(F)\right)$. Let $S$ be a Sylow 2-subgroup of $R$, and let $T^{*}$ be a Sylow 2-subgroup of $G$ which contains $S$. Then $N=$ $R \mathbf{N}_{N}(S)=\mathbf{C}_{G}(F) \mathbf{N}_{N}(S)$, so $N$ normalizes $\Phi(S) \cap F$. $\Phi(S) \cap F \leq \Phi\left(T^{*}\right) \cap F=W^{*}=$ $\left\{1, \omega^{*}, \xi^{*}, \eta^{*}\right\}$ where $\omega^{*} \sim \omega$, etc. Now we have that $\omega^{*}$ is the only one of 
$\omega^{*}, \xi^{*}, \eta^{*}$ which is the only member of its G-conjugacy class to lie in $F$. By Lemma 3(ii), $\xi^{*}$ and $\eta^{*}$ are not central in $\mathbf{N}_{G}(F)$. It follows that $\Phi(S) \cap F$ cannot be $W^{*},\left\langle\xi^{*}\right\rangle$, or $\left\langle\eta^{*}\right\rangle$, so $\Phi(S) \cap F \leq\left\langle\omega^{*}\right\rangle=\langle\omega\rangle$.

Therefore, $F \Phi(S) / \Phi(S) \cong F / F \cap \Phi(S)$ is elementary of order 8 or 16. As $S / \Phi(S)$ is elementary of order at most $16[12], S=\langle F, s\rangle$ for some $s$. Hence $\mathbf{0}_{2}\left(\mathbf{A}_{G}(F)\right)$ is cyclic, and cannot faithfully admit a 3-element of $\mathbf{A}_{G}(F)$. As $\mathbf{A}_{G}(F)$ is solvable, we get that $\mathbf{0}_{3}\left(\mathbf{A}_{G}(F)\right)>1$.

Since $\mathbf{A}_{G}(F)$ has a fixed point, $\mathbf{0}_{3}\left(\mathbf{A}_{G}(F)\right)=\langle\sigma\rangle$ is of order 3 , with $F=$ $[F, \sigma] \times \mathrm{C}_{F}(\sigma)$ and $[F, a], \mathrm{C}_{F}(\sigma)$ both of order 4 . The normalizer of such a $\langle\sigma\rangle$ in $L_{4}(2)$ has 2-part a four-group, so $\left|\mathbf{A}_{G}(F)\right|=12$ and $V$ acts nontrivially on $\mathbf{C}_{F}(\sigma)$ and $[F, \sigma]$. It follows that $W \cap \mathbf{C}_{F}(\sigma)=\langle\omega\rangle$, so $\mathbf{C}_{F}(\sigma)=\langle\omega, \gamma\rangle$ for some $\gamma$. Then there is $v \in V$ with

$$
\gamma^{v}=\gamma \omega ; \quad(\gamma[F, \sigma])^{v}=\gamma \omega[F, \sigma] .
$$

Now $\langle[F, \sigma], \omega\rangle\left\langle F\right.$, so there is $\delta \in \bigcap \cap(F-\langle[F, \sigma], \omega\rangle)$. Let $T_{1}$ be a Sylow 2-subgroup of $\mathbf{C}_{G}(\delta)$ containing $F$. Then Lemma $3(i)$ implies $W_{1}=\langle\omega, \delta\rangle$. But $\delta$ is conjugate to $\delta \omega$ by $\langle v, \sigma\rangle$. This contradiction establishes that $\mathbf{A}_{G}(F)$ acts fixed-point-freely on $F^{\#}$.

To show $A_{G}(F) \cong \Sigma_{3} \times \Sigma_{3}$, it will suffice to show that $\left|\mathbf{0}_{3}\left(A_{G}(F)\right)\right|=9$, since $A_{G}(F)$ contains a four-group and we know the structure of the normalizer in $L_{4}(2)$ of a group of order 9. If $\mathbf{0}_{2}\left(\mathbf{A}_{G}(F)\right)=1$, then $\mathbf{0}_{3}\left(\mathbf{A}_{G}(F)\right)$ must be a faithful module for a four-group, so must be of order 9 . Hence we will show $\mathbf{0}_{2}\left(\mathbf{A}_{G}(F)\right)=1$.

Let $K=\mathbf{0}_{2}\left(\mathbf{A}_{G}(F)\right)$. $\quad \mathbf{C}_{F}(K)=H$ is invariant under a Sylow 3-subgroup of $\mathbf{A}_{G}(F)$. If this Sylow 3-subgroup had nontrivial fixed-point-set $H_{0}$ on $H$, then $H_{0}$ would contain a fixed point for $\mathrm{A}_{G}(F)$. So $H$ is a four-group upon which some 3-element acts fixed-point-freely.

Let $S$ be a Sylow 2-subgroup of $\mathbf{N}_{G}(F)$, and let $T^{*}$ be a Sylow 2-subgroup of $G$ containing $S$. Then $V^{*} \triangleleft S$, so $K$ intersects the group induced on $F$ by $V^{*}$ nontrivially. If this intersection had order 4 , then $H=W^{*}=\mathrm{C}_{F}\left(V^{*}\right)$ and no 3-element could act nontrivially on $H$. Hence the intersection has order 2 , and is generated $\bmod \mathbf{C}_{G}(F)$ by some $v \in V^{*}$.

$W^{*} \leq \mathbf{C}_{F}(v)$. If $\mathbf{C}_{F}(v)=W^{*}$ then $\mathbf{C}_{F}(v)=H=W^{*}$, so $\mathbf{C}_{F}(v)$ contains $H$ with index 2. Therefore $[F, v]$ has order 2 .

We claim no fixed-point-free 3-element $\rho$ can occur in $\mathbf{N}_{G}(F)$. For if so, let $X$ be a $\rho$-invariant complement to $H$ in $F$. We can choose $\{r, s, t\}=H^{\#}$ and $\{x, y, z\}=X^{\#}$ so that the $\rho$-orbits of $F^{\#}$ are

$$
\{r, s, t\} ;\{x, y, z\} ;\{r x, s y, t z\} ;\{r z, s x, t y\} ;\{r y, s z, t x\} .
$$


Now $v, v^{\rho}$, and $v^{\rho^{2}}$ have as centralizers in $F$ the three groups of order 8 containing $H$. We may assume

$$
\begin{gathered}
\mathbf{C}_{F}(v)=\langle H, x\rangle, \quad \mathbf{C}_{F}\left(v^{\rho}\right)=\langle H, y\rangle, \quad \mathbf{C}_{F}\left(v^{\rho^{2}}\right)=\langle H, z\rangle ; \\
{[F, v]=\langle r\rangle, \quad\left[F, v^{\rho}\right]=\langle s\rangle, \quad\left[F, v^{\rho^{2}}\right]=\langle t\rangle .}
\end{gathered}
$$

Then $y^{v}=y r, z^{v}=z r ; z^{v v^{\rho}}=z r s=z t$, so all the elements of $F^{\#}$ except $r, s$, and $t$ are conjugate under $\langle v, \rho\rangle$. This is impossible as $F^{\#}$ contains three distinct $G$-classes.

It follows that the Sylow 3-subgroups of $\mathrm{A}_{G}(F)$ are generated by elements $\sigma$ with $[F, \sigma]=H$ and $\mathrm{C}_{F}(\sigma)=X$ say a complement to $H$ in $F$. Hence every conjugate of $v$ by an element of $\langle\sigma\rangle$ has the same centralizer. As the automorphism of $F$ induced by $v$ lies in the center of a Sylow 2-subgroup of $A_{G}(F)$, we get that $\mathbf{C}_{F}(v)$ is invariant under $\mathbf{A}_{G}(F)$. $W^{*} \leq \mathbf{C}_{F}(v)$, so the three $\langle\sigma\rangle$-orbits in $\mathrm{C}_{F}(v)$ are already distinct $G$-orbits, and so $\mathrm{C}_{F}(v)$ contains a fixed point of $\mathbf{A}_{G}(F)$. This contradiction completes the proof that $\mathbf{0}_{2}\left(\mathbf{A}_{G}(F)\right)=1$, and so finishes the proof of (ii).

(iii) Let $F$ be any $E_{16}$ of $T$ with $F=\langle F \cap \supsetneqq\rangle$. Let $W^{\#}=\{\omega, \xi, \eta\}$, let $F=A \times B$ where $A^{\#}=\operatorname{ccl}_{G}(\omega) \cap F$, and $A=\langle\omega, \alpha\rangle ; B^{\#}=\operatorname{ccl}_{G}(\xi) \cap F$, and $B$ $=\langle\xi, \beta\rangle$.

Let $R$ be any normal $\mathrm{Z}_{4} \times \mathrm{Z}_{4}$ of $T$. Then $R=\langle v, u\rangle$ where $v^{2}=\omega, u^{2}=$ $\xi$, $\alpha$ inverts $v$ and centralizes $u$, and $\beta$ inverts $u$ and centralizes $v$.

Proof. $\Omega_{1}(R)=W$. Since $\mathrm{C}_{R}(F)=W$ (Lemma 3(iii)), $R$ induces a full Sylow 2-subgroup of $A_{G}(F) \cong \Sigma_{3} \times \Sigma_{3}$. $A_{G}(F)$ contains automorphisms $\bar{\rho}, \bar{\sigma}$ such that

$$
[F, \bar{\rho}]=A, \quad \mathrm{C}_{F}(\bar{\rho})=B ; \quad[F, \bar{\sigma}]=B, \quad \mathrm{C}_{F}(\bar{\sigma})=A
$$

Also, there is $v \in R-W$ such that $\alpha^{v}=\alpha \omega$ and the automorphism of $F$ induced by $v$ centralizes $\bar{\sigma}$.

Let $K=\left\langle\mathrm{C}_{G}(F), v, \sigma\right\rangle$ where $\sigma \mathrm{C}_{G}(F)$ induces $\bar{\sigma}$ on $F . L=\left\langle\mathrm{C}_{G}(F), v\right\rangle \triangleleft$ $K$, so $K=L N_{K}(S)$, where $S=\langle v, F\rangle$ is a Sylow 2-subgroup of $L$ (Lemma 3(iii)). So we can find a 3-element $\sigma \in \mathrm{N}_{K}(S)$ such that $\sigma \mathrm{C}_{G}(F)$ induces $\bar{\sigma}$. $\sigma$ centralizes $S / F$ and $F / B$, hence $[S, \sigma]=[F, \sigma]=B$; so $[v, \sigma] \in B$, and $\left[v^{2}, \sigma\right]=$ $[v, \sigma]^{v}[v, \sigma] \epsilon\langle\xi\rangle$. But $v^{2}=\omega, \xi$, or $\eta$, and $\left[v^{2}, \sigma\right]=1$ if $v^{2}=\omega$, and is otherwise $\xi \beta$ or $\beta$. Hence we must have $\left[v^{2}, \sigma\right]=1$ and $v^{2}=\omega$. Since $v$ leaves $B$ invariant and centralizes $\bar{\sigma}, v$ must centralize $\beta$, as claimed.

We now fix a particular $F \leq T$ with $F \cong E_{16}$ and $F=\langle F \cap \$\rangle$. We take notation in $F$ so that 
Define

$$
\begin{aligned}
& W^{\#}=\{\omega, \xi, \eta\} ; \quad F=\left\langle W, \omega^{*}, \xi^{*}\right\rangle \text { where } \omega^{*} \sim \omega, \xi^{*} \sim \xi ; \\
& \operatorname{ccl}_{G}(\omega) \cap F=\left\{\omega, \omega^{*}, \omega \omega^{*}\right\} ; \quad \operatorname{ccl}_{G}(\xi) \cap F=\left\{\xi, \xi^{*}, \xi \xi^{*}\right\} ; \\
& \operatorname{ccl}_{G}(\dot{\eta}) \cap F=\text { the remaining set of nine involutions of } F .
\end{aligned}
$$

$$
\begin{aligned}
& I(\omega)=\{\gamma \in T-W: \gamma \sim \omega \text { and } W(\gamma)=\langle\omega\rangle\} ; \\
& I(\xi)=\{\gamma \in T-W: \gamma \sim \xi \text { and } W(\gamma)=\langle\xi\rangle\} ; \\
& H_{\omega}=\langle I(\omega)\rangle ; \quad H_{\xi}=\langle I(\xi)\rangle .
\end{aligned}
$$

(Here $W(\gamma)$ means $\{x \in W: y \sim \gamma x\}$.)

(iv) Let $R$ be any normal $\mathrm{Z}_{4} \times \mathrm{Z}_{4}$ of $T$; then all $\gamma \in I(\omega)$ induce the same automorphism of $R$.

Proof. By applying (iii) to $F$ and $R$, we get that $R=\langle v, u\rangle$ where $\langle v, W\rangle=$ $\mathbf{C}_{R}\left(\xi^{*}\right)$ and $\langle u, W\rangle$ is the set of elements of $R$ inverted by $\xi^{*}$.

Let $E=\left\langle\xi^{*}, \gamma, W\right\rangle$. In $E$, we have $\gamma, \omega, \gamma \omega \in \operatorname{ccl}_{G}(\omega) ; \xi, \xi, \xi \xi \in \operatorname{ccl}_{G}(\xi)$; $\eta, \xi^{*} \omega, \xi^{*} \eta \in \mathrm{ccl}_{G}(\eta) ; \gamma \xi, \gamma \eta$ are $R$-conjugate; $\gamma \xi^{*}, \gamma \xi^{*} \omega, \gamma \xi^{*} \xi, \gamma \xi^{*} \eta$ are $R$-conjugate. Now the sets of $G$-conjugate involutions of $E$ have sizes 3,3 , and 9 where the ones of size 3 are four-groups. Hence $\left|\mathrm{ccl}_{G}(\eta) \cap E\right| \neq 3$, so $\left|\mathrm{ccl}_{G}(\eta) \cap E\right|=9$ and $\gamma, \xi^{*}$ fulfill the conditions of $\alpha, \beta$ in (iii). Hence, by (iii) applied to $E$ and $R, \gamma$ inverts $\mathrm{C}_{R}\left(\xi^{*}\right)=\langle v, W\rangle$ and centralizes the set $\langle u, W\rangle$ of elements of $R$ inverted by $\xi^{*}$. This determines the action of $\gamma$ on $R$.

We now return to our usual normal $\mathrm{Z}_{4} \times \mathrm{Z}_{4}, V$, of $T$.

(v) $V \cap H_{\omega}$ contains $\lambda_{0}$ with $\lambda_{0}^{2}=\omega$.

Proof. By applying (iii) to $F$ and $V$, we get $V=\langle v, u\rangle$ where $v^{2}=\omega, u^{2}$ $=\xi, \omega^{*}$ inverts $v$ and centralizes $u$, and $\xi^{*}$ inverts $u$ and centralizes $v$.

Suppose some $y \in v \omega^{*} W$ is conjugate in $G$ to an element of $W$. Let $E=\left\langle W, \xi^{*}, \gamma\right\rangle$; then $E=\langle E \cap \supsetneqq\rangle$, and of the involutions of $E, \xi^{*}$ and $\xi^{*} \xi \epsilon \operatorname{ccl}_{G}(\xi), \xi^{*} \omega$ and $\xi^{*} \eta \epsilon$ $\operatorname{ccl}_{G}(\eta)$, while the elements of $\gamma \xi^{*} W$ are all conjugate. If no element of $\gamma W$ were conjugate to $\omega$, then $\operatorname{ccl}_{G}(\omega) \cap E=\{\omega\} \propto\{\omega\} \cup \gamma \xi^{*} W$, contrary to (iii). Hence, $\gamma z \sim \omega$ for some $z \in W$. The set $\{\omega\} \cup \gamma W \cup \gamma \xi^{*} W$ is not eligible as $\operatorname{ccl}_{G}(\omega) \cap E$ because the product of its members, which would be invariant under $A_{G}(E)$, is $\omega$. Hence

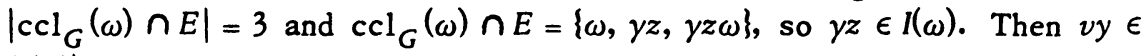
$\langle I(\omega)\rangle$ for some $y \in W$ and we may take $\lambda_{0}=v y$.

We will show, by transfer, that some $\gamma \in v \omega^{*} W$ is conjugate to an element of W. Assume the contrary.

First, $v$ is not a square in $\mathrm{C}_{T}(V)=C$. For if $t \in C$ had $t^{2}=v$, then

$$
\omega=\left[\omega^{*}, v\right]=\left[\omega^{*}, t^{2}\right]=\left[\omega^{*}, t\right]\left[\omega^{*}, t\right]^{t}
$$


But $\Omega_{3}(C) / V$ is central in $T$ (since operator-isomorphic to a subgroup of $W$ ), so $\left[\omega^{*}, t\right] \in V$, and we get

$$
\omega=\left[\omega^{*}, t\right]^{2} ; \quad\left[\omega^{*}, t\right]=v z \quad(z \in W) ; \quad\left(\omega^{*}\right)^{t}=\omega^{*} v z
$$

It follows that $C$ is Abelian of type $\left(4,2^{r}\right)$ for some $r \geq 2$. Hence every element of $\left[C, \omega^{*}\right]$ has the form $\left[x, \omega^{*}\right]$ for some $x \in C$, and similarly for $\xi^{*}$. As $\omega^{*} \chi \omega^{*} \xi$ and $\xi^{*} \chi \xi^{*} \omega,\left[C, \omega^{*}\right]$ and $\left[C, \xi^{*}\right]$ are cyclic. $\left[C, \omega^{*}\right] \leq$ the set of elements of $C$ inverted by $\omega^{*}$, which is $\langle v, w\rangle$; hence $\left[C, \omega^{*}\right]=\langle\omega\rangle$, and $[C$ : $\left.\mathbf{C}_{C}\left(\omega^{*}\right)\right]=2$, and $C=\langle v\rangle \times\langle y\rangle$, for some $y \in \mathbf{C}_{C}\left(\omega^{*}\right)$. As $\left|\mathbf{C}_{C}\left(\xi^{*}\right)\right|=8,\left[C, \xi^{*}\right]$ is cyclic of order $1 / 2|y|$, and is generated by $y^{2} w$ for some $w \in W$. It follows that $\xi^{*}$ inverts $y^{2}$, and then that $y^{\xi^{*}}=y^{-1} z$ for some $z \in W$.

Next, $\Phi(T) \leq\langle\omega, y\rangle$. For if $x \in T$ had $x^{2} \equiv v \bmod \langle\omega, y\rangle$, then

$$
\omega=\left[\omega^{*}, x^{2}\right]=\left[\omega^{*}, x\right]\left[\omega^{*}, x\right]^{x} .
$$

Since $\omega^{*}$ inverts $\left[\omega^{*}, x\right],\left[\omega^{*}, x\right] \in\langle v, W\rangle$, and by our assumption, $\left[\omega^{*}, x\right] \in W$. But then $\left[\omega^{*}, x\right]\left[\omega^{*}, x\right]^{x}=1 \neq \omega$; As $T^{2}=\Phi(T)$, we have $\Phi(T) \leq\langle\omega, y\rangle$.

Hence, $\left\langle\omega, y^{2}\right\rangle \leq \Phi(T) \leq\langle\omega, y\rangle$.

Suppose first that $\Phi(T)=\left\langle\omega, y^{2}\right\rangle$. Then the four-generator theorem of [12] gives $T=\left\langle v, y, \omega^{*}, \xi^{*}\right\rangle$. Let $M=\left\langle\omega, y, \omega^{*}, \xi^{*}\right\rangle$, a maximal subgroup of $T$; by transfer, $\omega^{*} v$ is conjugate to some element of $M$. If $z \neq 1$, then all the involutions of $M$ are $T$-conjugate to elements of $\omega^{*} W, \xi^{*} W, \omega^{*} \xi^{*} W$, or $W$, so $\omega^{*} v$ is conjugate to an element of $W$. Hence, $z=1$ and $\omega^{*} v$ is $G$-conjugate to $\xi^{*} y$, $\xi^{*} y \omega$, or $\omega^{*} \xi^{*} y$. Now

$$
\begin{aligned}
\mathbf{C}_{T}\left(\omega^{*} v\right) & =\left\langle\omega^{*} v\right\rangle \times\langle\omega\rangle \times\left\langle\xi^{*}, y\right\rangle ; \\
\mathbf{C}_{T}\left(\xi^{*} y\right) & =\mathbf{C}_{T}\left(\xi^{*} y \omega\right)=\left\langle\xi^{*} y\right\rangle \times\langle\xi\rangle \times\left\langle\omega^{*}, v\right\rangle ; \\
\mathbf{C}_{T}\left(\omega^{*} \xi^{*} y\right) & =\left\langle W, \omega^{*}, \xi^{*} y\right\rangle \cong E_{16^{*}}
\end{aligned}
$$

The unique involutions in the derived groups of $\mathbf{C}_{T}\left(\xi^{*} y\right)=\mathbf{C}_{T}\left(\xi^{*} y \omega\right)$ and $\mathrm{C}_{T}\left(\omega^{*} v\right)$ are not $G$-conjugate. Hence $\omega^{*} v \sim \omega^{*} \xi^{*} y$, and there is $g \in G$ with

$$
\left(\omega^{*} \xi^{*} y\right)^{8}=\omega^{*} v ; \quad E^{8}=\left\langle W, \omega^{*} v, y\right\rangle=F \quad \text { say }
$$

where $E=\mathbf{C}_{T}\left(\omega^{*} \xi^{*} y\right)$ and $\gamma$ is either $\xi^{*}$ or $\xi^{*} y$.

Now if $E=\langle E \cap B\rangle$, then all elements of $E^{\#}$ would be conjugate to elements of $W$; hence $E \cap Z=\left\langle W, \omega^{*}\right\rangle^{\#}$ and $|F \cap \bigcap|=7$.

If $\gamma=\xi^{*}$, then $F \cap Z=\left\langle W, \xi^{*}\right\rangle^{\#}$, so $\left\langle W, \omega^{*}\right\rangle^{8}=\left\langle W, \xi^{*}\right\rangle$. But this is impossible as $\left\langle W, \omega^{*}\right\rangle$ contains only one conjugate of $\xi$ while $\left\langle W, \xi^{*}\right\rangle$ contains three. 
If $\gamma=\xi^{*} y$, then, as $\xi^{*} y W \leq E-E \cap \not$, we have $F \cap Z=\left\langle W, \omega^{*} v y\right\rangle$, and $\left\langle W, \omega^{*}\right\rangle^{8}=\left\langle W, \omega^{*} v \gamma\right\rangle$. But $\omega^{*} v \gamma W$ is a single $T$-class and so some element of $W$ has five conjugates in $\left\langle W, \omega^{*} v \gamma\right\rangle$, which is contradicted in $\left\langle W, \omega^{*}\right\rangle$.

Suppose now that $\Phi(T)=\langle\omega, y\rangle$, so that there is $x \in T-\left\langle C, \omega^{*}, \xi^{*}\right\rangle$ with $x^{2} \equiv \omega^{r} y^{e}$ for some odd $e$. Then

$$
\left[\xi^{*}, y\right]^{e}=\left[\xi^{*}, y^{e}\right]=\left[\xi^{*}, x^{2}\right]=\left[\xi^{*}, x\right]\left[\xi^{*}, x\right]^{x} .
$$

Now $\xi^{*}\left[\xi^{*}, x\right]=\left(\xi^{*}\right)^{x} \in I(\xi)$, so $\omega^{*}$ centralizes $\xi^{*}\left[\xi^{*}, x\right]$ and hence $\left[\xi^{*}, x\right]$. So $\left[\xi^{*}, x\right] \epsilon\langle\omega, y\rangle$. Since $\left\langle\omega, x^{2}\right\rangle=\langle\omega, y\rangle, x$ centralizes $\left[\xi^{*}, x\right]$, and

$$
\left[\xi^{*}, y\right]^{e}=\left[\xi^{*}, x\right]^{2}
$$

Now $\left[\xi^{*}, y\right]=y^{2} z$ where $z \in W$, so $\left[\xi^{*}, x\right]^{2}=\left(y^{2} z\right)^{e}$. But $\left[\xi^{*}, x\right] \epsilon\langle\omega, y\rangle=$ $\Phi(T)$, so $z \in \Omega_{1}(\langle y\rangle)$ and $\left[\xi^{*}, x\right]=y^{f} t$, where $t \in W$ and $y^{f}$ may be taken as $y^{e}$ or $y^{e} y_{1}$ where $\left\langle y_{1}\right\rangle=\Omega_{2}(\langle y\rangle)$. (If $|y|=4$ then we can always take $y^{f}=y^{e}$, since $\left[\xi^{*}, x\right]^{2}$ $=\left[\xi^{*}, y\right]^{*}$ implies that $\left[\xi^{*}, x\right]$ is a generator for $\left.C_{\text {. }}\right)$ Also, $\xi^{*}$ inverts $y$.

Now $\left(\omega^{*}\right)^{x}=\omega^{*}$ or $\omega^{*} \omega$. $(x v)^{2} \equiv x^{2} \bmod W$, so $(x v)^{2}$ has the same form as $x^{2}$ and we may replace $x$ by $x v$, if necessary, to get the following relations between $x, \xi^{*}, \omega^{*}$, and $v$ :

$$
\left[\omega^{*}, x\right]=1 \text {. }
$$

$x^{2}=\omega^{r} y^{e}=y$ say, by choice of $y \in \mathbf{C}_{C}\left(\omega^{*}\right)$; then $\left[\xi^{*}, x\right]=y t$ for some $t \in W$, since $\left[\xi^{*}, x\right]^{2}=\left[\xi^{*}, x^{2}\right]=\left[\xi^{*}, y\right]=y^{2}$.

$x \notin C$, so $x$ does not centralize $v$ since then $x$ would centralize $V$. Also, $x \omega^{*} \notin C$, so $[v, x]=\xi$ or $\xi \omega$. As $x$ centralizes $\omega^{*},\left(x \omega^{*}\right)^{2}=x^{2}$, so we can replace $x$ by $x \omega^{*}$, if necessary, to get the following relations for $\left\langle v, x, \xi^{*}, \omega^{*}\right\rangle$ :

$$
\begin{aligned}
& v^{x}=v \xi, \quad y^{x}=y, \quad x^{2}=y ; \\
& v^{\xi^{*}}=v, \quad y^{\xi^{*}}=y^{-1} ; \quad v^{\omega^{*}}=v^{-1}, \quad y^{\omega^{*}}=y ; \\
& {\left[\omega^{*}, \xi^{*}\right]=1 ;} \\
& {\left[\omega^{*}, x\right]=1, \quad\left[\xi^{*}, x\right]=y \omega^{j} \xi^{k}, \text { for some } j, k .}
\end{aligned}
$$

As $\Phi(T)=\langle\omega, y\rangle$, the four-generator theorem of $[12]$ implies that $T=\left\langle v, x, \omega^{*}, \xi^{*}\right\rangle$.

If we can find a maximal subgroup $M$ of $T$ all of whose involutions are $G$. conjugate to elements of $W$, then by transfer $\omega^{*} v$ is conjugate to an element of $W$. We establish that such an $M$ can be found by simply examining the squares of the elements of the nonidentity cosets of $\Phi(T)$ in $T$, so as to see which cosets have the property that all their involutions are conjugate to elements of $W$. 
We take coset representatives $\nu, \omega^{*}, \xi^{*}, x, \nu \omega^{*}, \nu \xi^{*}, v x, \omega^{*} \xi^{*}, \omega^{*} x, \xi^{*} x$, $v \omega^{*} \xi^{*}, v \omega^{*} x, v \xi^{*} x, \omega^{*} \xi^{*} x$, and $v \omega^{*} \xi^{*} x$ for the nonidentity cosets of $\Phi(T)=\langle\omega, y\rangle$ in $T$. Using that $\left(r \omega y^{i}\right)^{2}=\left(r y^{i}\right)^{2}$ for any $r \in T$, we get the following table for these squares:

$$
\begin{aligned}
& \text { * }\left(v y^{i}\right)^{2}=\omega y^{2 i} \\
& \text { * }\left(\omega^{*} \xi^{*} y^{i}\right)^{2}=1 \\
& \text { * }\left(\omega^{*} y^{i}\right)^{2}=y^{2 i} \\
& \left(\xi^{*} x y^{i}\right)^{2}=\omega^{j} \xi^{k} \\
& \text { * }\left(\xi^{*} y^{i}\right)^{2}=1 \\
& \text { * }\left(\omega^{*} x y^{i}\right)^{2}=y^{1+2 i} \\
& \text { * }\left(x y^{i}\right)^{2}=y^{1+2 i} \\
& \left(v \omega^{*} \xi^{*} y^{i}\right)^{2}=1 \\
& \left(v \omega^{*} y^{i}\right)^{2}=y^{2 i} \\
& \text { * }\left(v \omega^{*} x y^{i}\right)^{2}=\xi y^{1+2 i} \\
& \text { * }\left(v \xi^{*} y^{i}\right)^{2}=\omega \\
& \left(\nu \xi^{*} x y^{i}\right)^{2}=\omega^{j+1} \xi^{k+1} \\
& \text { * }\left(v x y^{i}\right)^{2}=\omega \xi y^{1+2 i} \\
& \left(\omega^{*} \xi^{*} x y^{i}\right)^{2}=\omega^{j} \xi^{k} \\
& \left(v \omega^{*} \xi^{*} x y^{i}\right)^{2}=\omega^{j} \xi^{k+1} .
\end{aligned}
$$

The cosets marked $*$ are those all of whose involutions are conjugate to elements of $W$ for all values of $j$ and $k$.

If follows that if $\xi^{k} \neq 1$, then $M=\left\langle\omega^{*}, \xi^{*}, x\right\rangle$ has all its involutions conjugate to elements of $W$; while if $\xi^{k}=1, M=\left\langle\omega^{*}, \xi^{*} v x\right\rangle$ does.

(vi) $\left\langle H_{\omega}, W\right\rangle$ contains no normal $\mathbf{Z}_{4} \times \mathbf{Z}_{4}$ of $T$.

Proof. Let $H=\left\langle H_{\omega}, W\right\rangle$ and suppose $R \leq H$ where $R$ is a normal $\mathrm{Z}_{4} \times \mathrm{Z}_{4}$ of T. $W=\Phi(R)$, so $H=\langle I(\omega), W\rangle=\langle I(\omega)\rangle$. All elements of $I(\omega)$ are congruent $\bmod \mathbf{C}_{T}(R)$, by (iv), so

$$
\begin{aligned}
H & =\left\langle x \omega^{*}: x \in \mathrm{C}_{T}(R) \text { and } x \omega^{*} \in I(\omega)\right\rangle \\
& =\left\langle\left\langle x: x \in \mathrm{C}_{T}(R) \text { and } x \omega^{*} \in I(\omega)\right\rangle, \omega^{*}\right\rangle .
\end{aligned}
$$

Now $\omega^{*}$ normalizes $\left\{x: x \in \mathrm{C}_{T}(R)\right.$ and $\left.x \omega^{*} \in I(\omega)\right\}$, so $\left\langle x: x \in \mathrm{C}_{T}(R)\right.$ and $x \omega^{*}$ $\epsilon I(\omega)\rangle$ has index 2 in $H$, and $H \cap \mathrm{C}_{T}(R)=\left\langle x: x \in \mathrm{C}_{T}(R)\right.$ and $\left.x \omega^{*} \in I(\omega)\right\rangle$. Hence $H \cap \mathrm{C}_{T}(R)$ is generated by elements which $\omega^{*}$ inverts. Applying Lemma $7(\mathrm{~b})$ to $H \cap \mathrm{C}_{T}(R)$, we get that $\omega^{*}$ inverts $R$, so $W\left(\omega^{*}\right)=W$, contrary to $\omega^{*} \in I(\omega)$.

The same argument as in the proof of (i.v) now gives that $\left\langle H_{\omega}, W\right\rangle$ is the direct product of a dihedral group of order $\geq 8$ and a group of order 2. We can take notation as follows:

(vii) $\left\langle H_{\omega}, W\right\rangle=\langle\pi\rangle \times\left\langle\lambda, \omega^{*}\right\rangle$, where $\pi \in W-\langle\omega\rangle$ and $\lambda \omega^{*}, \omega^{*} \in I(\omega) .\left\langle\operatorname{ccl}{ }_{G}(\omega)\right.$ $\cap T\rangle=\left\langle\lambda, \omega^{*}\right\rangle$, so $\left\langle\lambda, \omega^{*}\right\rangle \triangleleft T$. $|\lambda| \geq 4$.

$\left\langle H_{\xi}, W\right\rangle=\langle\rho\rangle \times\left\langle\mu, \xi^{*}\right\rangle$ where $\rho \in W-\langle\xi\rangle$ and $\mu^{*}, \xi^{*} \in I(\xi) .\left\langle\mu, \xi^{*}\right\rangle=\left\langle\operatorname{ccl}{ }_{G}{ }^{(\xi)}\right.$ $\cap T\rangle$ so is normal in $T .|\mu| \geq 4$. 

$\left.\omega^{*}, \xi^{*}\right\rangle$.

Since $\langle\lambda\rangle$ and $\langle\mu\rangle \triangleleft T, \mathbf{A}_{T}(V)$ is induced by $\left\langle\omega^{*}, \xi^{*}\right\rangle$, and hence $T=\left\langle C_{T}(V)\right.$,

Let $C=\mathrm{C}_{T}(V), \Delta=\langle\lambda, \mu\rangle$. Then

(viii) (a) $\Omega_{1}(C / \Delta)$ is central in $T$.

(b) Suppose $\rho^{2}=\lambda, \sigma^{2}=\mu$, and $r^{2}=\lambda \mu$, where $\rho, \sigma, r \in C$. Then $\left[\omega^{*}, \rho\right]=$ $\lambda$ or $\lambda \omega,\left[\omega^{*}, \tau\right]=\lambda$ or $\lambda \omega,\left[\omega^{*}, \sigma\right]=1$ or $\omega$; and $\left[\xi^{*}, \rho\right]=1$ or $\xi,\left[\xi^{*}, \tau\right]=\mu$ or $\mu \xi,\left[\xi^{*}, \sigma\right]=\mu$ or $\mu \xi$.

Proof. (a) Suppose $\rho$ and $\sigma \in C$ with $\rho^{2}=\lambda$ and $\sigma^{2}=\mu$. Then $\rho \Delta=\{x \epsilon$ $\left.C: x^{2} \in \lambda \Delta^{2}\right\}$ is $T$-invariant, and so is $\mu \Delta$.

(b) Suppose $x=\rho, \sigma$, or $r$; then $\left[\omega^{*}, x\right] \in H_{\omega} \cap C=\langle\lambda\rangle$. Now $\rho^{2}=\lambda$, so $\rho$ centralizes $\left[\omega^{*}, \rho\right] . \tau^{2}=\lambda \mu$ and $r$ normalizes $\langle\lambda\rangle$ and $\langle\mu\rangle$, so must centralize them both, since $\langle\lambda\rangle \cap\langle\mu\rangle=1$, and so $r$ centralizes $\left[\omega^{*}, r\right]$. Hence

$$
\begin{aligned}
& \lambda^{2}=\left[\omega^{*}, \lambda\right]=\left[\omega^{*}, \rho^{2}\right]=\left[\omega^{*}, \rho\right]^{2}, \text { so }\left[\omega^{*}, \rho\right] \equiv \lambda \bmod \langle\omega\rangle, \\
& \lambda^{2}=\left[\omega^{*}, \lambda \mu\right]=\left[\omega^{*}, \tau^{2}\right]=\left[\omega^{*}, \tau\right]^{2}, \text { so }\left[\omega^{*}, \tau\right] \equiv \lambda \bmod \langle\omega\rangle .
\end{aligned}
$$

Also,

$$
1=\left[\omega^{*}, \mu\right]=\left[\omega^{*}, \sigma^{2}\right]=\left[\omega^{*}, \sigma\right]\left[\omega^{*}, \sigma\right]^{\sigma} \text {. }
$$

Now $\left[\omega^{*}, \sigma\right] \epsilon \Delta$, and $\sigma^{2}$ centralizes $\Delta$, so $\left[\omega^{*}, \sigma\right]^{\sigma} \equiv\left[\omega^{*}, \sigma\right] \bmod W($ Lemma $6(g))$. Hence

$$
1 \equiv\left[\omega^{*}, \sigma\right]^{2} \bmod \langle\omega\rangle
$$

and so $\left[\omega^{*}, \sigma\right]=\lambda_{0}^{k}$ for some $k$, where $\lambda_{0}$ generates $\Omega_{2}(\langle\lambda\rangle)$.

Similarly $\left[\xi^{*}, \sigma\right] \equiv \mu \bmod \langle\xi\rangle,\left[\xi^{*}, \tau\right] \equiv \mu \bmod \langle\xi\rangle$, and $\left[\xi^{*}, \rho\right]=\mu_{0}^{n}$.

We need only prove that $k$ and $n$ are even. For this, we use that every $x \epsilon$ $\xi^{*} \omega^{*} C$ has $C_{C}(x)=W$, so $x^{2} \in C$ implies $x^{2} \in W$. Let $x=\xi^{*} \omega^{*} \rho$. Then

$$
\begin{aligned}
x^{2} & =\left(\omega^{*} \rho\right)^{\xi^{*}}\left(\omega^{*} \rho\right)=\omega^{*} \rho \mu_{0}^{-n} \omega^{*} \rho \equiv \rho^{-1} \mu_{0}^{n} \rho \bmod \langle\omega\rangle \\
& \equiv \mu_{0}^{n} \bmod \langle\omega\rangle,
\end{aligned}
$$

as $\rho \in C$. Hence $\mu_{0}^{n} \in W$. Similarly $\lambda_{0}^{k} \in W$, and (viii) is proved.

(ix) $C / \Delta$ is elementary (of order $\leq 4$ ).

Proof. We will show that $\lambda, \mu, \lambda \mu$ cannot be fourth powers in $C$. First suppose $\delta \in C, \delta^{4}=\lambda \mu$, and let $r=\delta^{2}$. Then by (viii),

$$
\lambda \equiv\left[\omega^{*}, \delta^{2}\right]=\left[\omega^{*}, \delta\right]^{2}\left[\omega^{*}, \delta, \delta\right] \bmod \langle\omega\rangle .
$$

Now if $\left[\omega^{*}, \delta\right]$ has order $2^{k}$, then $\left[\omega^{*}, \delta\right]^{2 k-2} \in V \leq Z(C)$, so $\left[\omega^{*}, \delta, \delta\right]$ has order $\leq 2^{k-2}$ by Lemma $6(c)$. Hence by Lemma $6(d),\left[\omega^{*}, \delta\right]^{2}\left[\omega^{*}, \delta, \delta\right]$ has order $2^{k-1}$. Hence 


$$
|\lambda|=2^{k-1}=1 / 2\left|\left[\omega^{*}, \delta\right]\right| \cdot
$$

But $\left[\omega^{*}, \delta\right] \in H_{\omega} \cap C=\langle\lambda\rangle$, so this is impossible.

Now suppose $\delta \in C, \delta^{4}=\lambda$, and let $\rho=\delta^{2}$. Then

$$
\lambda \equiv\left[\omega^{*}, \rho\right]=\left[\omega^{*}, \delta\right]^{2}\left[\omega^{*}, \delta, \delta\right] \bmod \langle\omega\rangle
$$

has order $1 / 2\left|\left[\omega^{*}, \delta\right]\right|$, a contradiction as before. By symmetry, (ix) holds.

(x) Let $X=\left\langle\omega, \omega^{*}\right\rangle$ or $\left\langle\xi, \xi^{*}\right\rangle$. Then the Sylow 2-subgroups of $\mathbf{C}_{G}(X)$ are of the form $Q \times X$ where $Q$ is dihedral or semidihedral.

Proof. Take $X=\left\langle\xi, \xi^{*}\right\rangle$. By (ii), there are 3-elements $\gamma$ and $\delta \in \mathbf{N}_{G}(F)$ where $\gamma$ cycles $\left\langle\omega, \omega^{*}\right\rangle$ and centralizes $X$, and $\delta$ cycles $X$ and centralizes $\left\langle\omega, \omega^{*}\right\rangle$. Let $R=\mathrm{C}_{G}(X)$; then $\delta$ acts on $R$, and by the Frattini argument there is a $\delta$-invariant Sylow 2-subgroup $S$ of $R$.

$F / X$ is a four-group of $R / X$, and the normalizer in $R / X$ of $F / X$ is $\left(\mathbf{N}_{G}(F) \cap \mathbf{C}_{G}(X)\right) / X=\left\langle\lambda_{0}, F\right\rangle / X$, where $\lambda_{0}$ generates $\Omega_{2}(\langle\lambda\rangle)$. This implies that the Sylow 2-subgroups of $R / X$ are of maximal class, and they are dihedral or semidihedral since $F / X$ is a four-group.

We will show that $R / X$ has Sylow 2-subgroups of order $\geq 16$, by exhibiting an element of order $\geq 8$ in $R / X$. Namely, if $|\lambda| \geq 8$ or if $C$ contains $\rho$ with $\rho^{2}$ $=\lambda$ (so that $\rho$ or $\rho \mu_{0}$ centralizes $X$ ), then $\lambda$ or $\rho$ or $\rho \mu_{0}$ has order $\geq 8 \bmod X$. So we may assume $|\lambda|=4$ and no such $\rho$ exists. If there is no $r \in C$ with $r^{2}=$ $\lambda \mu$, it follows from (viii) that $\left\langle\omega^{*}, W\right\rangle$ is a normal $E_{8}$ of $T$. So we may assume that $|\lambda|=4$ and $C=\langle\lambda, \mu, \tau\rangle$ where $r^{2}=\lambda \mu$ and $|\mu|=2^{n} \geq 4$.

$D=\left\langle\omega^{*}, \lambda\right\rangle \times\left\langle\xi^{*}, \mu\right\rangle$ is a maximal subgroup of $T$, and its exponent is $2^{n}=$ $|\mu|$. The elements of $\tau \Delta, \tau \omega^{*} \Delta, \tau \xi^{*} \Delta$, and $\tau \omega^{*} \xi^{*} \Delta$ (where $\left.\Delta=\langle\lambda, \mu\rangle\right)$ have the following orders and squares, respectively: $2^{n+1} ; 2^{n+1} ; 8$, with squares $\lambda \xi^{j}$ or $\lambda \omega \xi^{j}$; and 4 or 2 , with square $\omega^{i} \xi^{j}$, where in (viii) we have $\left[\omega^{*}, \tau\right]=\lambda \omega^{i}$ and $\left[\xi^{*}, \tau\right]=\mu \xi^{j}$.

Let $\nu$ be the transfer homomorphism from $G$ to $T / D$. Then

$$
\nu(\tau)=\prod g^{\tau} g^{-1} \prod g \tau^{2} g^{-1} \cdots \prod g \tau^{2^{n-2}} g^{-1} \prod g \tau^{2^{n-1}} g^{-1} \prod g \tau^{2^{n}} g^{-1} D,
$$

in the usual way. $|\tau|=2^{n+1}$, so all the factors in $\Pi g r g^{-1}$ lie in $T-D$. Since $\nu(r)=1$, some factor of order 8,4 , or 2 must lie in $T-D$. This means that either $r^{2^{n-2}} \sim \tau \xi^{*} x(x \in \Delta), r^{2^{n-1}} \sim \tau \omega^{*} \xi^{*} x(x \in \Delta)$, or $r^{2^{n}} \sim \tau \omega^{*} \xi^{*} x(x \in \Delta)$. We will obtain a contradiction from each possibility.

Suppose $\tau^{2 n-2} \sim \tau \xi^{*} x(x \in \Delta)$. Then the squares of these elements are conjugate, so, using that $\lambda \sim_{T} \lambda \omega$, we have $r^{2 n-1} \sim \lambda \xi^{j}$. If $n=2$, this means $\lambda \mu$ $\sim \lambda \xi^{j}$. Now $\mathbf{C}_{T}\left(\lambda \xi^{j}\right)=\left\langle C, \xi^{*}\right\rangle$ and $\mathbf{C}_{T}(\lambda \mu)=C$, so there is $g \in G$ with $(\lambda \mu)^{g}=$ $\lambda \xi^{j}$ and $C^{g} \leq\left\langle C, \xi^{*}\right\rangle$. $C$ is the only Abelian maximal subgroup of $\left\langle C, \xi^{*}\right\rangle$, so $g$ normalizes $C$. But $\mathbf{A}_{G}(C)$ is a 2 -group, so is covered by $T$, and $\lambda_{\mu}$ is not 
$T$-conjugate to $\lambda \xi^{j}$. If $n \geq 3$, then we have $\lambda_{0} \omega^{2 n-3} \sim \lambda \xi^{j}$, where $\mu_{0}$ generates $\Omega_{2}(\langle\mu\rangle)$. There is $g \in G$ with $\left(\mu_{0} \omega^{2^{n-3}}\right)^{8}=\lambda \xi^{j}$ and $\left\langle C, \omega^{*}\right\rangle^{8} \leq\left\langle C, \xi^{*}\right\rangle$; as above, $g$ normalizes $C$, which is impossible.

Now suppose $\tau^{2 n-1} \sim \tau \omega^{*} \xi^{*} x(x \in \Delta)$. Then $\tau^{2^{n-1}} \sim \tau \omega^{*} \xi^{*}=a$ say; $\mathbf{C}_{T}(a)$ $=\langle a, W\rangle=\langle a\rangle \times\langle\omega\rangle=A$ say.

Suppose $n=2$. Then we have $\lambda \mu \sim a$, and there is $g \in G$ with $a^{\boldsymbol{g}}=\lambda_{\mu}$ and $A^{g} \leq C$, so $A^{\mathcal{B}}=\langle\lambda \mu, W\rangle$. Now $\mathrm{N}_{T}(A)=\langle A, \lambda, \mu\rangle$ and $\mathrm{N}_{T}(\langle\lambda \mu, W\rangle)=T$, so there is $b \in G$ such that $\lambda^{b} \in T$ and $\lambda^{b}$ conjugates $\lambda \mu$ to $\lambda \mu \omega$. But the elements of $T$ which conjugate $\lambda \mu$ to $\lambda \mu \omega$ are those of $C \omega^{*}$, and none of these have squares $\sim \lambda^{2}=\omega$.

Suppose $n \geq 3$. Then we have $\mu_{0} \omega^{2^{n-3}} \sim a$, and there is $g \in G$ with $a^{8}=$ $\mu_{0} \omega^{2^{n-3}}$ and $A^{\overline{8}} \leq\left\langle C, \omega^{*}\right\rangle$. Now the $G$-conjugates of $\omega$ in $\left\langle C, \omega^{*}\right\rangle$ are conjugate in $\left\langle C, \omega^{*}\right\rangle$ to $\omega$ or $\omega^{*}$; so we may assume $\omega^{8}=\omega$ or $\omega^{*}$, and $A^{8}=B$ or $L$, where $B=\left\langle\mu_{0} \omega^{2^{n-3}}, \omega\right\rangle$ and $L=\left\langle\mu_{0} \omega^{2 n-3}, \omega^{*}\right\rangle$.

All elements of order 4 in $A$ are $G$-conjugate (in fact, $T$-conjugate). But $\mu_{0} \chi_{G} \mu_{0} \omega$; for if so, there is $g \in G$ with $\mu_{0}^{g}=\mu_{0} \omega$ and $\left\langle C, \omega^{*}\right\rangle^{g}=\left\langle C, \omega^{*}\right\rangle . C$ is the unique Abelian maximal subgroup of $\left\langle C, \omega^{*}\right\rangle$, so $C^{g}=C^{\prime}$; but $T$ covers $\mathbf{N}_{G}(C)$, and $\mu_{0} \chi_{T} \mu_{0} \omega$.

It follows that $A \not B$, and so $A^{g}=L$. Now $\left|\mathbf{N}_{G}(L)\right| \geq\left|\mathbf{N}_{T}(A)\right|$, but $\mathbf{N}_{T}(L)$ only inverts $L$, whereas $\mathbf{N}_{T}(A)$ induces a four-group on $A$. Hence, $\mathbf{N}_{T}(L)$ cannot be a Sylow 2-subgroup of $\mathrm{N}_{G}(L)$, and there must be some $Y \leq T, Y \cong Z_{4} \times$ $\mathbf{Z}_{2}$ (with the proper $G$-fusion pattern of involutions), such that $A \sim L \sim Y$ and $\mathbf{N}_{T}(Y)$ is a Sylow 2-subgroup of $\mathbf{N}_{G}(Y)$.

We shall now find $Y . Y \leq D$, for otherwise $Y \sim_{T} A$. The elements of $D$ with squares $\sim \xi=\left(\mu_{0} \omega^{2 n-3}\right)^{2}$ are $T$-conjugate to $\mu_{0}, \mu_{0} \omega$, or $\omega^{*} \mu_{0}$, and $D \cap$ $\operatorname{ccl}_{G}(\omega)=\{\omega\} \cup \omega^{*}\langle\lambda\rangle$. Taking $Y$ to have $\left\langle\mu_{0}\right\rangle,\left\langle\mu_{0} \omega\right\rangle$, or $\left\langle\omega^{*} \mu_{0}\right\rangle$ as a direct factor, we get the following possibilities (up to $T$-conjugacy) for $Y$ :

$$
\begin{aligned}
\mu_{0}:\left\langle\mu_{0}, \omega\right\rangle & =B \text {, or }\left\langle\mu_{0}, \omega^{*}\right\rangle . \\
\mu_{0} \omega:\left\langle\mu_{0} \omega, \omega\right\rangle & =B \text {, or }\left\langle\mu_{0} \omega, \omega^{*}\right\rangle . \\
\omega^{*} \mu_{0}:\left\langle\omega^{*} \mu_{0}, \omega\right\rangle & =Y_{1} ;\left\langle\omega^{*} \mu_{0}, \omega^{*}\right\rangle=Y_{2} ; \text { or }\left\langle\omega^{*} \mu_{0}, \omega^{*} \omega\right\rangle=Y_{3} .
\end{aligned}
$$

$Y \notin B$, since $A \not B$. If $Y \notin\left\{Y_{1}, Y_{2}, Y_{3}\right\}$, then $\left|\mathbf{N}_{T}(Y)\right|=2^{n+3}=\left|\mathbf{N}_{T}(L)\right|$, violating that $\mathbf{N}_{T}(L)$ is not a Sylow 2-subgroup of $\mathbf{N}_{G}(L)$; for the same reason, $Y \notin\left\{Y_{2}, Y_{3}\right\}$. So $Y=\left\langle\omega^{*} \mu_{0}, \omega\right\rangle$.

$A \sim Y$ and $\mathbf{N}_{T}(Y)$ is a Sylow 2-subgroup of $\mathbf{N}_{G}(Y)$. Therefore, there is $b \epsilon$ $G$ with $A^{b}=Y$ and $\mathbf{N}_{T}(A)^{b} \leq \mathbf{N}_{T}(Y) . \mathbf{N}_{T}(A)=\left\langle A, \lambda, \mu_{0}\right\rangle$ and $\mathbf{N}_{T}(Y)=\left\langle\left\langle\omega^{*}, \mu\right.\right.$, $\left.\omega\rangle, \xi^{*}, \lambda\right\rangle$, where $\mu_{0}$ inverts $A ; \mathbf{C}_{T}(Y)=\left\langle\omega^{*}, \mu, \omega\right\rangle$ and $\xi^{*}$ inverts $Y$. Therefore $\mu_{0}^{b} \epsilon\left\langle\omega^{*}, \mu, \omega\right\rangle \xi^{*}$; but $\left\langle\omega^{*}, \mu, \omega\right\rangle \xi^{*}$ consists of involutions. 
We may now suppose $r^{2^{n}} \sim r \omega^{*} \xi^{*}=a$ say. $C_{T}(a)=\langle a, W\rangle=A$ say is elementary of order 8 .

Suppose $n=2$. Then we have $\eta=\omega \xi \sim a$, and there is $g \in G$ with $a^{g}=\eta$ and $A^{8} \leq T$. We claim $A^{8} \leq D$; namely, if $A^{8} \leq D$, then $A^{8} \leq\left\langle W, \omega_{0}, \xi_{0}\right\rangle=E$ say, where $\operatorname{ccl}_{G}(\omega) \cap E=\left\{\omega, \omega_{0}, \omega \omega_{0}\right\}$ and $\operatorname{ccl}_{G}(\xi) \cap E=\left\{\xi, \xi_{0}, \xi \xi_{0}\right\}$. If $\omega^{8}$ $=\omega$, then $(a \omega)^{8}=\eta \omega=\xi$, but $a \omega \sim a \sim \eta$. Hence, by $T$-conjugacy, we may assume $\omega^{8}=\omega_{0}$ and (similarly) $\xi^{8}=\xi_{0}$, so $A^{8}=\left\langle\eta, \omega_{0}, \xi_{0}\right\rangle$. But then $\mathrm{N}_{T}(A)=$ $\left\langle A, \lambda, \mu_{0}\right\rangle$ and $\mathrm{N}_{T}\left(A^{8}\right)=\mathrm{C}_{T}\left(A^{8}\right)=E$, and neither $\mathrm{N}_{T}(A)$ nor $\mathrm{N}_{T}\left(A^{8}\right)$ is isomorphic to a subgroup of the other, so there must be some $Y \leq T, Y \cong E_{8}$, with $A \sim A^{8} \sim Y$ and $\mathbf{N}_{T}(Y)$ a Sylow 2-subgroup of $\mathbf{N}_{G}(Y)$, so that $\left|\mathbf{N}_{T}(Y)\right|>\left|\mathbf{N}_{T}(A)\right|$. The argument above shows that $Y \underline{\notin} D$, and so $Y \sim_{T} A$, violation $\left|N_{T}(Y)\right|>$ $\left|\mathbf{N}_{T}(A)\right|$. Hence, $A^{8}\left\lfloor\underline{D}\right.$, and we may assume $A^{8}=A$. But then $\omega$ and $\xi$ are the only members of their $G$-classes in $A$, so $\omega^{8}=\omega, \xi^{8}=\xi$, and $a^{8}=\eta=\omega \xi$, which is impossible.

Suppose $n \geq 3$. Then $\xi \sim a$, and there is $g \in G$ with $a^{g}=\xi$ and $A^{g} \leq T$. $\left|\operatorname{ccl}_{G}(\xi) \cap A\right|=5$, so $A^{8} \not D$, and we may assume $A^{8}=A$. But then, as before, we get $\omega^{8}=\omega, \eta^{8}=\eta$, and $a^{8}=\xi=\omega \eta$, which is impossible.

We now have that the Sylow 2-subgroups of $R / X$ are dihedral or semidihedral of order $\geq 16$. Also, we have the 3-elements $\gamma$ and $\delta$ of $\mathrm{N}_{G}(X)$, and a $\delta$-invariant Sylow 2-subgroup $S$ of $\mathrm{C}_{G}(X)=R$, as described at the beginning of the proof of $(x)$.

$\left\langle\lambda, \omega^{*}\right\rangle \leq R$, so there is $x \in R$ with $\left\langle\lambda, \omega^{*}\right\rangle^{x} \leq S$. $(\omega X)^{x} \in R / X$ lies in $\Phi(S / X)$, so is the unique central involution of $S / X$. The element $\gamma$ (or $\gamma^{-1}$ ) of $\mathrm{N}_{G}(F) \cap R$ sends $\omega$ to $\omega^{*}$, and so $\gamma^{x} X \in R / X$ sends $\omega^{x} X$ to $\left(\omega^{*}\right)^{x} X$.

Let $Y / X=\mathbf{0}_{2},(R / X)$, and let $M / Y$ be a minimal normal subgroup of $R / Y$. Write $\bar{R}$ for $R / Y$. Then $\bar{S} \cong S / X$ has center $\left\langle\bar{\omega}^{x}\right\rangle$, so $\omega^{x} \in M$. Since $M \triangleleft R$ and $\gamma^{x} \in R,\left(\omega^{*}\right)^{x} \in M$ and $M \cap S$ has index at most 2 in $S$. As $\bar{S}$ is dihedral or semidihedral of order $\geq 16$, the Sylow 2-subgroups of $\bar{M}$ are non-Abelian indecomposable and $\bar{M}$ is a non-Abelian simple group. Therefore, $\bar{M}^{(\infty)}=\bar{M}$, i.e., $M^{(\infty)} Y=M Y=M$. $R / M$ has odd or twice-odd order, so $(R / M)^{(\infty)}=1$ [7], i.e., $R^{(\infty)} Y=M$.

We now show that $R^{(\infty)} \cap X=1$. Namely, $R^{(\infty)}$ and $X$ are both $\delta$-invariant, and $\delta$ acts irreducibly on $X$, so $R^{(\infty)} \cap X \neq 1$ if and only if $X \leq R^{(\infty)} . R^{(\infty)}$ is in the kernel of the transfer $\nu: R \rightarrow S / S^{\prime}$. But for $y \in X$,

$$
\nu(y)=\prod\left(g y g^{-1}\right) S^{\prime}=y s^{\prime},
$$

since $X \leq \mathrm{Z}(R)$ (where $g$ runs over a transversal to $S$ in $R$ ). As $S / X$ is dihedral or semidihedral and $X \leq Z(S), S^{\prime}$ is generated by a single commutator, so $S^{\prime}$ is 
cyclic and there is $y \in X-S^{\prime}$. This $y$ is not in the kernel of $\nu$, hence not in $R^{(\infty)}$, so $R^{(\infty)} \cap X=1$.

Let $S_{1}=S \cap M$, and $P=S_{1} \cap R^{(\infty)}$, so $S_{1}=P \times X . P$ is $\delta$-invariant and is dihedral or semidihedral of order $\geq 8$, so is centralized by $\delta$. If $S_{1}=S$, then $S$ $=P \times X$ and $(x)$ holds. Suppose $\left[S: S_{1}\right]=2$. Then $|S / P|=8$ and $\delta$ acts fixedpoint-freely on $X P / P \leq S / P$, so $S / P$ is elementary with one fixed point under $\delta$; so $\mathrm{C}_{S}(\delta)\left\lfloor P\right.$. Now $S=\mathrm{C}_{S}(\delta)[S, \delta]$ and $\mathrm{C}_{S}(\delta)>P,[S, \delta] \geq X, X \cap \mathrm{C}_{S}(\delta)=1$; so $X=[S, \delta]$ and $S=\mathrm{C}_{S}(\delta) \times X$, where $\mathrm{C}_{S}(\delta) \cong S / X$ is dihedral or semidihedral.

(xi) Take $\rho$ and $\sigma$ as in (viii); the $\left[\rho, \xi^{*}\right]=\left[\sigma, \omega^{*}\right]=1$.

Proof. $\left[\rho, \xi^{*}\right]=1$ or $\xi$ by (viii). Suppose $\left[\rho, \xi^{*}\right]=\xi$. Then $\rho \mu_{0}$ centralizes $\xi^{*}$ (where $\mu_{0}$ generates $\Omega_{2}((\mu))$ ), and

$$
\left[\rho \mu_{0}, \omega^{*}\right]=\lambda^{-1} \text { or } \lambda^{-1} \omega ; \quad\left(\rho \mu_{0}\right)^{2}=\lambda \xi
$$

Hence $\langle\lambda, \xi\rangle \leq \Phi\left(\mathrm{C}_{T}\left(\xi^{*}\right)\right)$. But $(x)$ gives that $\Phi\left(\mathrm{C}_{T}\left(\xi^{*}\right)\right)$ is cyclic.

We will now show that $T$ is a direct product of dihedral and semidihedral groups. This is true if $C=\langle\lambda, \mu\rangle$, for then $T=H_{\omega} \times H_{\xi}$. If $[C:\langle\lambda, \mu\rangle]=2$, then (xi) implies that we may assume $C=\langle\lambda, \mu, \tau\rangle$ where $r^{2}=\lambda \mu$. Hence $r$ centralizes $\lambda$ and $\mu$ (since it normalizes $\langle\lambda\rangle$ and $\langle\mu\rangle)$, and $T=\left\langle\lambda, \mu, \tau, \omega^{*}, \xi^{*}\right\rangle$, where $r^{2}=\lambda \mu,\left[\omega^{*}, \tau\right]=\lambda \omega^{i},\left[\xi^{*}, \tau\right]=\mu \xi^{j}$, and $r$ centralizes $\langle\lambda, \mu\rangle$. By symmetry, we may assume $|\lambda| \leq|\mu|=2^{n} \geq 4$. Then $T$ is the same group encountered in the proof of $(x)$ while showing that $R / X$ contained elements of order $\geq 8$, except that $|\lambda|$ need not be 4 . The transfer argument used in the proof of $(x)$ to eliminate this $T$ does not require $|\lambda|=4$, and applies in the present case (if one reads $\lambda_{0}$ for $\lambda$ where necessary). Hence, this $T$ cannot occur.

We may now assume $[C: \Delta]=4$, where $\Delta=\langle\lambda, \mu\rangle$. Let $\rho$ and $\sigma \in C$ with $\rho^{2}=\lambda$ and $\sigma^{2}=\mu$. To show $T$ is a direct product of dihedral and semidihedral groups, we need only show $[\rho, \sigma]=1$, by $(x i)$. Now $[\rho, \sigma] \in V$ since $\left[\rho^{2}, \sigma^{2}\right]=$ 1, by Lemma 6(c). But

$$
\left(\left(\xi^{*} \sigma\right)\left(\omega^{*} \rho\right)\right)^{2}=\left(\xi^{*} \sigma\right)^{2}\left(\omega^{*} \rho\right)^{\xi^{*} \sigma^{*} \rho} \equiv \omega^{*} \rho^{\sigma} \omega^{*} \rho \equiv[\rho, \sigma] \bmod w
$$

Since $\mathbf{C}_{V}\left(\xi^{*} \sigma \omega^{*} \rho\right)=W$, we must have $[\rho, \sigma] \in W$. So

$$
\begin{aligned}
T & =\left\langle\rho, \sigma, \omega^{*}, \xi^{*}\right\rangle, \text { where } \rho^{2}=\lambda, \sigma^{2}=\mu ;\left[\omega^{*}, \sigma\right]=\left[\xi^{*}, \rho\right]=1 ; \\
{\left[\omega^{*}, \rho\right] } & =\lambda \omega^{i},\left[\xi^{*}, \sigma\right]=\mu \xi^{k} ;[\rho, \sigma]=y \in W .
\end{aligned}
$$

The squares of the elements of $T$ are 


$$
\begin{array}{rlrl}
C^{2} & =\Delta & \left(\xi^{*} \rho \Delta\right)^{2} & =\lambda\left\langle\lambda^{2}\right\rangle \\
\left(\omega^{*} \Delta\right)^{2} & =\left\langle\mu^{2}\right\rangle & \left(\xi^{*} \sigma \Delta\right)^{2} & =\xi^{k}\left\langle\lambda^{2}\right\rangle \\
\left(\omega^{*} \rho \Delta\right)^{2} & =\omega^{i}\left\langle\mu^{2}\right\rangle & \left(\xi^{*} \rho \sigma \Delta\right)^{2} & =\xi^{k} y \lambda\left\langle\lambda^{2}\right\rangle \\
\left(\omega^{*} \sigma \Delta\right)^{2} & =\mu\left\langle\mu^{2}\right\rangle & \left(\omega^{*} \xi^{*} \Delta\right)^{2} & =1 \\
\left(\omega^{*} \rho \sigma \Delta\right)^{2} & =\omega^{i} y \mu\left\langle\mu^{2}\right\rangle & \left(\omega^{*} \xi^{*} \rho \Delta\right)^{2} & =\omega^{i} \\
\left(\xi^{*} \Delta\right)^{2} & =\left\langle\lambda^{2}\right\rangle & \left(\omega^{*} \xi^{*} \sigma \Delta\right)^{2} & =\xi^{k} \\
\left(\omega^{*} \xi^{*} \rho \sigma \Delta\right)^{2}= & \omega^{i} \xi^{k} y
\end{array}
$$

We claim all involutions of $T$ are conjugate to elements of $W$. This follows by transfer if $\omega^{i}$ or $\xi^{k} \neq 1$, as all the involutions of the maximal subgroups $\langle C$, $\left.\omega^{*}\right\rangle$ or $\left\langle C, \xi^{*}\right\rangle$ are then conjugate to elements of $W$. If $\omega^{i}=\xi^{k}=1$, transfer gives $\omega^{*} \rho \sim \xi^{*} \sigma \not$ the elements of $W$. But then, supposing $\left|\mathbf{C}_{T}\left(\xi^{*} \sigma\right)\right| \geq$ $\left|\mathbf{C}_{T}\left(\omega^{*} \rho\right)\right|, \mathbf{C}_{T}\left(\omega^{*} \rho\right)$ is conjugate to a subgroup of $\mathbf{C}_{T}\left(\xi^{*} \sigma\right)$, contrary to $\langle\xi\rangle=$ $\Omega_{1}\left(\Phi\left(\mathbf{C}_{T}\left(\omega^{*} \rho\right)\right)\right),\langle\dot{\omega}\rangle=$ ditto for $\xi^{*} \sigma$.

We now claim $\omega^{i}=\omega$ and $\xi^{k}=\xi$. For if $\omega^{i}=1$, then $\left\langle W, \omega^{*} \rho, \xi^{*}\right\rangle$ satisfies the hypotheses of (ii). The classes in $G$ of the elements of $W$ and $\xi^{*} W$ are known, and $\omega^{*} \rho \xi^{*} W$ is contained in a single class, $\omega^{*} \rho \sim \omega^{*} \rho \omega$, and $\omega^{*} \rho \xi \sim$ $\omega^{*} \rho \eta$. It follows that $\omega^{*} \rho z \sim \omega$ for some $z \in W$, and $\omega^{*} \rho z \xi \not \omega$. This gives $\omega^{*} \rho z \in I(\omega)$, whence $\rho z \in H_{\omega}=\left\langle\omega^{*}, \lambda\right\rangle$, a contradiction.

By symmetry, we need only deal with the cases $y=\omega$ and $y=\eta$.

Suppose $y=\eta$. Then $\omega^{*} \xi^{*} \rho \sigma=\left(\omega^{*} \rho\right)\left(\xi^{*} \sigma\right)=\alpha$ say is an involution, so is conjugate to some element of $W . \mathrm{C}_{T}(\alpha)=\langle\alpha, \omega\rangle \times\left\langle\omega^{*} \rho \lambda_{0} \mu_{0}\right\rangle$, where $\left(\omega^{*} \rho \lambda_{0} \mu_{0}\right)^{2}$ $=\eta$. Let $\alpha^{8} \in W$ and $\mathbf{C}_{T}(\alpha)^{8} \leq T$. Then $\eta^{8} \in \Phi(T)$, so $\eta^{8}=\eta$, and $(a \eta)^{8}=$ $a^{8} \eta$. Therefore, $\alpha^{8} \psi(\alpha \eta)^{8}$. But $\alpha \sim \alpha \eta$ in $T$ (e.g., by $\lambda_{0} \mu_{0}$ ).

For the case $y=\omega$, we need that $\mu_{0} \not \mu_{0} \omega$. If $\mu_{0} \sim \mu_{0} \omega$, there is $g \in G$ with $\mu_{0}^{g}=\mu_{0} \omega$ and $\mathbf{C}_{T}\left(\mu_{0}\right)^{g}=\mathbf{C}_{T}\left(\mu_{0}\right) . \Phi\left(\mathbf{C}_{T}\left(\mu_{0}\right)\right)=C^{2}=\Delta$, and by examining squares in cosets of $\Delta$ we get that $C^{B}=C$. Now $A_{G}(C)$ is a 2-group, so $\mathbf{A}_{G}(C)=\mathbf{A}_{T}(C)$; but $\mu_{0} \chi_{T} \mu_{0} \omega$.

Now suppose $y=\omega$. Let $M=\left\langle\mu, \rho, \omega^{*}, \xi^{*}\right\rangle$; then $T-M$ contains no involutions, so by transfer, $\xi^{*} \sigma$ is conjugate to an element of $M$. Since $\left(\xi^{*} \sigma\right)^{2}=$ $\xi$, we get $\xi^{*} \sigma \sim \mu_{0}, \mu_{0} \omega$, or $\omega^{*} \mu_{0}$. Now if $\xi^{*} \sigma \sim \mu_{0}$ or $\mu_{0} \omega$, then there is $g \epsilon$ $G$ with $\left(\xi^{*} \sigma\right)^{g}=\mu_{0}$ or $\mu_{0} \omega$ and $\mathbf{C}_{T}\left(\xi^{*} \sigma\right)^{g} \leq \mathbf{C}_{T}\left(\mu_{0}\right)$. $\mathbf{C}_{T}\left(\xi^{*} \sigma\right)=\left\langle\omega^{*}, \lambda\right\rangle \times\left\langle\xi^{*} \sigma\right\rangle$, and $\mathrm{C}_{T}\left(\mu_{0}\right)=\left\langle C, \omega^{*}\right\rangle$. The unique four-group of $\Phi\left(\mathrm{C}_{T}\left(\xi^{*} \sigma\right)\right)$ must go under $g$ to the unique four-group of $\mathrm{C}_{T}\left(\mu_{0}\right)$, i.e., $W^{g}=W$. But $\xi^{*} \sigma$ is conjugate in $T$ to $\xi^{*} \sigma z$ for every $z \in W$ (namely, $\left(\xi^{*} \sigma\right)^{\rho}=\xi^{*} \sigma \omega,\left(\xi^{*} \sigma\right)^{\mu_{0}}=\xi^{*} \sigma \xi$ ) and $\mu_{0} z \not$ $\mu_{0} z \omega$ for $z \in W$.

Hence $\xi^{*} \sigma \sim \omega^{*} \mu_{0} \chi \mu_{0}$ and $\mu_{0} \omega$. Indeed, the elements of $T$ that can be $G$-conjugate to $\xi^{*} \sigma$ are represented up to $T$-conjugacy by $\omega^{*} \mu_{0}, \omega^{*} \xi^{*} \sigma$, and 
$\omega^{*} \xi^{*} \rho \sigma$. The latter two have centralizers in $T$ of order $2^{4}$. Now

$$
\begin{aligned}
\mathbf{C}_{T}\left(\xi^{*} \sigma\right) & =\left\langle\omega^{*}, \lambda\right\rangle \times\left\langle\xi^{*} \sigma\right\rangle, \\
\mathbf{C}_{T}\left(\omega^{*} \mu_{0}\right) & =\left\langle W, \sigma, \omega^{*} \mu_{0}\right\rangle=\left\langle W, \sigma, \omega^{*}\right\rangle=\left\langle\omega^{*}\right\rangle \times\langle\omega\rangle \times\langle\sigma\rangle .
\end{aligned}
$$

It follows that $\mathrm{C}_{T}(y)$ is a Sylow 2 subgroup of $\mathrm{C}_{G}(y)$ where $y$ is either $\xi^{*} \sigma$ or $\omega^{*} \mu_{0}$. Hence one of $\mathrm{C}_{T}\left(\xi^{*} \sigma\right)$ and $\mathrm{C}_{T}\left(\omega^{*} \mu_{0}\right)$ is isomorphic to a subgroup of the other. But this is false, since the only Abelian rank 3 subgroups of $\mathbf{C}_{T}\left(\xi^{*} \sigma\right)$ are of type $(2,2,4)$ and $\mathrm{C}_{T}\left(\omega^{*} \mu_{0}\right)$ is of type $\left(2,2,2^{r}\right)$ for $r \geq 3$.

This completes the proof of Theorem B.

\section{REFERENCES}

1. J. Alperin, Centralizers of Abelian normal subgroups of pogroups, J. Algebra 1 (1964), 110-113. MR 29 \#4800.

2. J. Alperin, R. Brauer and D. Gorenstein, Finite groups with quasi-dihedral and wreathed Sylow 2-subgroups, Trans. Amer. Math. Soc. 151 (1970), 1-261. MR 44 \#1724.

3. N. Blackburn, Generalizations of certain elementary theorems on p-groups, Proc. London Math. Soc. (3) 11 (1961), 1-22. MR 23 \#A208.

4. R. Brauer, Some applications of the theory of blocks of characters of finite groups. II, J. Algebra 1 (1964), 307-334. MR 30 \#4836.

5. R. Carter and P. Fong, The Sylow 2-subgroups of the finite classical groups, J. Algebra 1 (1964), 139-151. MR 29 \#3548.

6. P. Chabot, Groups whose Sylow 2-subgroups have cyclic commutator groups. I, II, J. Algebra 19 (1971), 21-30; J. Algebra 21 (1972), 312-320.

7. W. Feit and J. Thompson, Solvability of groups of odd order, Pacific J. Math. 13 (1963), 775-1029. MR $29 \# 3538$.

8. G. Glauberman, Central elements in core-free groups, J. Algebra 4 (1966), 403420. MR $34 \# 2681$.

9. D. Gorenstein and J. Walter, The characterization of finite groups with dihedral Sylow 2-subgroups. I, II, III, J. Algebra 2(1965), 85-151, 218-270, 334-393. MR 31 $\# 1297 \mathrm{a}, \mathrm{b} ; 32 \# 7634$.

10. M. Hall, The theory of groups, Macmillan, New York, 1959. MR 21 \#1996.

11. B. Huppert, Endliche Gruppen. I, Die Grundlehren der math. Wissenschaften, Band 134, Springer-Verlag, Berlin and New York, 1967. MR 37 \#302.

12. A. MacWilliams, On 2-groups with no normal Abelian subgroups of rank 3, and their occurrence as Sylow 2-subgroups of finite simple groups, Trans. Amer. Math. Soc. 150 (1970), 345-408. MR $43 \# 2071$.

13. J. G. Thompson, Nonsolvable finite groups all of whose local subgroups are solvable, Bull. Amer. Math. Soc. 74 (1968), 383-437. MR $37 \# 6367$. ENGLAND

DEPARTMENT OF PURE MATHEMATICS, CAMBRIDGE UNIVERSITY, CAMBRIDGE,

Current address: 27 Overbrook Drive, Cheltenham, Gloucestershire, England 